\title{
Aplicações do cálculo estocástico à análise complexa
}

Rogério de Assis Medeiros

DISSERTAÇÃO APRESENTADA

$\mathrm{AO}$

INSTITUTO DE MATEMÁTICA E ESTATÍSTICA

DA

UNIVERSIDADE DE SÃO PAULO

PARA

OBTENÇÃO DO TÍTULO

$\mathrm{DE}$

MESTRE EM CIÊNCIAS

Programa: Matemática Aplicada

Orientador: Prof. Dr. Edson de Faria

Durante o desenvolvimento deste trabalho o autor recebeu auxílio financeiro do $\mathrm{CNPq}$

São Paulo, março de 2012 


\section{Aplicações do cálculo estocástico à análise complexa}

Este dissertação contém as correções e alterações sugeridas pela Comissão Julgadora durante a defesa realizada por Rogério de Assis Medeiros em 5/3/2012.

O original encontra-se disponível no Instituto de Matemática e Estatística da Universidade de São Paulo.

Comissão Julgadora:

- Prof. Dr. Edson de Faria (orientador) - IME-USP

- Prof. Dr. Adílson Simonis - IME-USP

- Prof. Dr. Nestor Felipe Caticha Alfonso - IF-USP 


\section{Agradecimentos}

Gostaria de agradecer a minha família, ao meu orientador Prof. Dr. Edson de Faria (IME-USP), aos professores e funcionários do IME, FEA e IMPA, aos professores que ministraram disciplinas no mestrado: Pedro Paulo Serpa Schirmer (IME-USP), Jorge Manuel Sotomayor Tello (IME-USP), Carlos Alberto de Bragança Pereira (IME-USP), Marcos Eugênio (FEA-USP), Frank Michael Forger (IME-USP), Leonardo Pellegrini (IME-USP), Élvia Mureb Sallum (IME-USP), José de Oliveira Siqueira (FEA-USP) e Adílson Simonis (IME-USP), aos professores da minha banca de qualificação Antonio Luiz Pereira (IME-USP) e Clodoaldo Grota Ragazzo (IME-USP), aos colegas e/ou professores Arlane Pereira (IME-USP), Eduardo Tengan (USP-São Carlos), Evandro Makyiama de Melo (IME-USP) e Fábio Niski (Courant Institute) que muito contribuíram com conversas, sugestões, críticas e comentários e também aos professores membros da minha banca examinadora. 


\section{Dedicatória:}

Dedico este trabalho aos meus pais, Sebastião Joaquim de Medeiros e Naide de Assis Medeiros, que muito lutaram para que fosse possível que eu conseguisse chegar aonde estou agora e poder prosseguir em minha trajetória. 


\section{Resumo}

\section{Aplicações do cálculo estocástico à análise complexa}

Nesta dissertação desenvolvemos o Cálculo Estocástico para provar teoremas clássicos de Análise Complexa, em particular, o pequeno teorema de Picard.

Palavras-chave: Integral Estocástica, Fórmula de Itô, Movimento Browniano, Análise Complexa, Teorema de Lévy 


\begin{abstract}
Applications of stochastic calculus to complex analysis

In this dissertation we develop the Stochastic Calculus for to prove classical theorems in Complex Analysis, in particular, the little Picard's theorem.
\end{abstract}

Keywords: Stochastic Integral, Ito's Formula, Brownian Motion, Complex Analysis, Lévy's Theorem 


\section{Sumário}

1 Introdução 10

2 Análise Complexa $\quad 12$

2.1 Preliminares . . . . . . . . . . . . . . . . . . 12

2.2 Teoremas Importantes . . . . . . . . . . . . . . . . . . . 14

2.3 Funções Harmônicas . . . . . . . . . . . . . . . . . . . . . . . 15

3 Noções Básicas de Probabilidade $\quad 17$

3.1 Preliminares . . . . . . . . . . . . . . . . . . 17

3.1 .1 Independência . . . . . . . . . . . . . . . . 21

3.1.2 Variáveis Aleatórias Gaussianas . . . . . . . . . . 23

3.1.3 Esperança Condicional . . . . . . . . . . . . . 25

4 Noções Básicas de Processos Estocásticos 27

4.1 Preliminares . . . . . . . . . . . . . . . . . . 27

4.2 Tempos de Parada . . . . . . . . . . . . . . . . . . . . 28

4.3 Propriedade de Markov . . . . . . . . . . . . . . . . . 30

4.3.1 Definições . . . . . . . . . . . . . . . 30

4.4 Martingais . . . . . . . . . . . . . . . . . . 30

4.4.1 Definições . . . . . . . . . . . . . . . 30

4.4 .2 Parada Opcional . . . . . . . . . . . . . . . 31

4.4 .3 Desigualdades de Doob . . . . . . . . . . . . . 34

4.4.4 Teoremas de Convergência para Martingais . . . . . . . 35

4.4.5 Decomposições de Supermartingais . . . . . . . . . . . 39

4.4.6 Variação Quadrática . . . . . . . . . . . . . . 45

4.5 Movimento Browniano . . . . . . . . . . . . . . . 47

4.5.1 Preliminares . . . . . . . . . . . . . 47

4.5.2 O Movimento Browniano é um Processo Markoviano . 51

4.5.3 Leis $0-1 \ldots \ldots \ldots \ldots$. . . . . . . . . . . 56

4.5.4 Propriedade Forte de Markov para o Movimento Browniano . . . . . . . . . . . . . . 57

4.5.5 O Movimento Browniano é um Martingal . . . . . . . 58

5 Cálculo Estocástico $\quad 61$

5.1 Integrais Estocásticas . . . . . . . . . . . . . . . 61

5.1 Construção . . . . . . . . . . . . . . . . . 61

5.1 .2 Extensões . . . . . . . . . . . . . . . 67

5.1 .3 Fórmula de Itô . . . . . . . . . . . . . . . . 68

5.1 .4 Aplicações . . . . . . . . . . . . . . . . . . . 73 
5.1.5 Representações Martingais . . . . . . . . . . . . 78

5.1.6 Mudança de Medida e o Teorema de Suporte . . . . . . 82

6 Cálculo Estocástico Complexo 86

6.1 Cálculo Estocástico Complexo . . . . . . . . . . . . . . 86

6.2 Invariância Conforme . . . . . . . . . . . . . . . . . . . 89

6.3 Aplicações . . . . . . . . . . . . . . . . . . . . . . . . . 92

6.3.1 Alguns Teoremas Interessantes . . . . . . . . . . . . . 92

6.3.2 "Pequeno"Teorema de Picard . . . . . . . . . . . . . 94

$\begin{array}{lll}7 & \text { Conclusão } & 97\end{array}$

$\begin{array}{ll}\text { Referências } & 98\end{array}$

$\begin{array}{ll}\text { Índice Remissivo } & 100\end{array}$ 


\section{Lista de Figuras}

1 Planos complexos domínio e imagem de $P(z) \ldots . . . . . .993$

2 Plano complexo com conjuntos $A_{i} \ldots \ldots$. . . . . . 95 


\section{Introdução}

The triumphant vindication of bold theories - are these not the pride and justification of our life's work?

Sherlock Holmes, The Valley of Fear

Sir Arthur Conan Doyle (1859-1930)

I have a difficult task ahead of me and I have dedicated my whole life to it.

K, The Castle

Franz Kafka (1883-1924)

A Teoria de Probabilidades é um rico e vasto campo da Matemática, sua fundamentação se dá por meio da Análise, principalmente por meio da Teoria da Medida.

Neste trabalho procuramos explorar um pouco desta interação entre Análise e Probabilidades no sentido oposto, fazendo uso de uma ferramenta probabilística sofisticada, o Cálculo Estocástico, para provar alguns teoremas clássicos em Análise Complexa.

No segundo capítulo enunciamos alguns conceitos e teoremas importantes de Análise Complexa que serão usados no capítulo 5.

No terceiro capítulo começamos a partir de conceitos e resultados fundamentais em Probabilidade, apresentamos aquele que será o protagonista desta dissertação, o movimento browniano e então exploramos algumas de suas propriedades (em especial, o fato de que o movimento browniano é um martingal e satisfaz a propriedade de Markov).

No quarto capítulo desenvolvemos o Cálculo Estocástico (no caso real) propriamente dito, com ênfase no movimento browniano. Aqui apresentamos um resultado fundamental: o Lema de Itô.

No quinto capítulo, o movimento browniano bidimensional é visto como movimento browniano complexo, e assim temos o Cálculo Estocástico Complexo, que nos permite provar o Teorema de Lévy, que é o resultado principal para 
a conexão entre o Cálculo Estocástico e a Análise Complexa.

É neste capítulo portanto que conectamos as idéias contidas no capítulo 2 com a teoria desenvolvida nos capítulos 3 e 4. Assim estamos em condições de provar resultados clássicos como o "pequeno"teorema de Picard.

No último e sexto capítulo mencionamos alguns desenvolvimentos que as idéias aqui apresentadas tomam em direções mais avançadas.

Entre minhas contribuições pessoais estão a organização e disposição do texto (a parte relativa ao Cálculo Estocástico Complexo é pouco encontrada na literatura e inexistente em língua portuguesa), além disso, a clarificação de diversos conceitos e a construção de algumas demonstrações é de minha autoria. 


\section{Análise Complexa}

(Regarding $\sqrt{-1}$ ): ... we can repudiate completely and which we can abandon without regret because one does not know what this pretended sign signifies nor what sense one ought to attribute to it.

Augustin-Louis Cauchy (1789-1857)

Neste capítulo vamos apresentar as definições e resultados básicos de Análise Complexa necessários para o desenvolvimento deste trabalho. O material aqui apresentado é clássico e mesmo os resultados mais avançados fazem parte de um primeiro ou segundo cursos de pós-graduação em variáveis complexas, sendo assim seremos breves em nossa apresentação. Neste capítulo seguiremos de perto [lins][10] e [rudin][16], que são ótimas referências para este assunto.

\subsection{Preliminares}

Seja $\mathbb{C}$ o conjunto dos números complexos e $U$ um subconjunto aberto. Seja $f: U \longrightarrow \mathbb{C}$ uma função contínua. Dizemos que $f$ é holomorfa em $z_{0} \in U$, se existe o limite

$$
f^{\prime}\left(z_{0}\right)=\lim _{h \longrightarrow 0} \frac{f\left(z_{0}+h\right)-f\left(z_{0}\right)}{h} .
$$

O número complexo $f^{\prime}\left(z_{0}\right)$ é chamado de derivada de $f$ em $z_{0}$. Se $f$ for holomorfa em todos os pontos de um subconjunto $X$ de $U$, diremos que $f$ é holomorfa em $X$. No caso em que $X$ coincide com $\mathbb{C}$ diremos que $f$ é inteira.

(2.1) Teorema: Sejam $U$ um subconjunto aberto dos números complexos e $f: U \longrightarrow \mathbb{C}$ uma função, onde $f(z)=f(x+i y)=f(x, y)=u(x, y)+i v(x, y)$. São equivalentes as seguintes propriedades:

(i) f é holomorfa em $U$; 
(ii) Todo ponto $z_{0} \in U$ é centro de uma bola na qual $f$ admite um desenvolvimento em série de potências de $z-z_{0}$, isto é, para cada $z_{0} \in U$, existe $r>0$ tal que se $\left|z-z_{0}\right|<r$, a série $\sum_{n=0}^{\infty} a_{n}\left(z-z_{0}\right)^{n}$ é convergente com soma $f(z)$. Ou seja, localmente $f$ é a soma de uma série de potências (assim, na teoria de variáveis complexas, dizer que uma função é holomorfa é o mesmo que dizer que ela é analítica);

(iii) A função $f(z)=u(x, y)+i v(x, y)$ é diferenciável, como função de $x e$ $y$, para todo ponto $(x, y)=z \in U$ e estão satisfeitas as condições de CauchyRiemann:

$$
\begin{aligned}
& \frac{\partial u}{\partial x}=\frac{\partial v}{\partial y} e \\
& \frac{\partial u}{\partial y}=-\frac{\partial v}{\partial x}
\end{aligned}
$$

(2.2) Definições: Seja $f$ uma função analítica num aberto $U \subset \mathbb{C}$. Dizemos que $z_{0} \in \mathbb{C}-U$ é uma singularidade isolada de $f$ se existe $r>0$ tal que para todo $z \neq z_{0} \operatorname{com}\left|z-z_{0}\right|<r, f$ está definida e é analítica em z. Em símbolos, fazendo $B_{r}\left(z_{0}\right)=\left\{z \in \mathbb{C}:\left|z-z_{0}\right|<r\right\}$ temos que $z_{0}$ é singularidade isolada se $B_{r}\left(z_{0}\right)-\left\{z_{0}\right\} \subset U$.

Sejam $f: U \rightarrow \mathbb{C}$ uma função analítica e $z_{0} \in \mathbb{C}-U$ uma singularidade isolada de $f$. Considere o conjunto $B_{r}\left(z_{0}\right)-\left\{z_{0}\right\} \subset U$, cuja existência é garantida por definição, tome o desenvolvimento em série de Laurent neste conjunto:

$$
f(z)=\sum_{n=-\infty}^{+\infty} a_{n}\left(z-z_{0}\right)^{n}
$$

Temos três possibilidades:

(i) $a_{n}=0$ para todo $n<0$. Neste caso diremos que $z_{0}$ é uma singularidade removível de $f$. Isto significa que $f$ se estende a uma função analítica em $z_{0}$, 
colocando-se $f\left(z_{0}\right)=a_{0}$. A série de Laurent de $f$ neste caso, será chamada de série de Taylor de $f$ em $z_{0}$;

(ii) Existe $k>0$ tal que $a_{-k} \neq 0$ e $a_{n}=0$ para todo $n<-k$. Neste caso diremos que $z_{0}$ é um polo de ordem $n$ de $f$, ou simplesmente um polo de $f$;

(iii) No caso em que o desenvolvimento de Laurent possui uma infinidade de termos não nulos com potências negativas de $z-z_{0}$ diremos que $z_{0}$ é uma singularidade essencial de $f$.

\subsection{Teoremas Importantes}

A seguir alguns teoremas importantes de Análise Complexa, alguns dos quais serão provados no capítulo 5, por meio do Cálculo Estocástico.

(2.3) Princípio do Máximo Módulo: Se D é um domínio limitado e $f$ é analítica em $D$ e contínua em $\bar{D}$, então $\sup _{\bar{D}}|f|=\sup _{\partial D}|f|$.

(2.4) Teorema da Aplicação Aberta: Seja $f: U \longrightarrow \mathbb{C}$ uma função analítica não constante, onde $U \subset \mathbb{C}$ é aberto e conexo. Então $f$ é aberta, isto é, para todo aberto $W \subset U, f(W)$ é aberto.

(2.5) Teorema Fundamental da Álgebra: Seja $P(z)$ um polinômio não constante em $z$. Então existe pelo menos um $z_{0}$ tal que $P\left(z_{0}\right)=0$, ou seja, todo polinômio complexo não constante possui pelo menos uma raiz. 
(2.6) Teorema de Liouville: Toda função $f$ inteira não constante é não limitada.

O próximo teorema é uma generalização do teorema de Liouville, mas antes de enunciá-lo, vamos olhar um pouco para a função $e^{z}$. A função $e^{z}$ é uma função inteira que omite o valor zero em sua imagem. Note que a série para $e^{z}$ converge absolutamente em todo ponto $z \in \mathbb{C}$ e portanto $e^{z}$ é inteira.

A convergência absoluta desta série permite-nos concluir que $e^{z} e^{w}=e^{z+w}$ e $e^{-z}=1 / e^{z}$ assim, se $e^{z}$ tivesse um zero na sua imagem teria um polo também, contradição.

(2.7) "Pequeno"Teorema de Picard: Se f é uma função inteira e se existirem dois números complexos que não estão na imagem de $f$, então $f$ é constante, ou seja, a imagem de $f$ omite no máximo um ponto se $f$ é uma função inteira não constante.

Note que este resultado é o melhor possível por causa do exemplo dado por $f(z)=e^{z}$.

\subsection{Funções Harmônicas}

(2.11) Definições: Se $f: \mathbb{C} \longrightarrow \mathbb{C}$ é diferenciável então definimos os operadores:

$$
\begin{aligned}
& \bar{\partial} f=\frac{\partial}{\partial \bar{z}} f=\frac{1}{2}\left(\frac{\partial f}{\partial x}+i \frac{\partial f}{\partial y}\right) \\
& \partial f=\frac{\partial}{\partial z} f=\frac{1}{2}\left(\frac{\partial f}{\partial x}-i \frac{\partial f}{\partial y}\right)
\end{aligned}
$$

Seja $f: U \longrightarrow \mathbb{C}$ uma função de classe $C^{2}$, onde $U \subset \mathbb{C}$ é um aberto. Dizemos que $f$ é harmônica se

$$
\frac{\partial^{2} f}{\partial x^{2}}+\frac{\partial^{2} f}{\partial y^{2}} \equiv 0 \Longleftrightarrow \Delta f \equiv 0
$$


em $U$, onde $\Delta=\frac{\partial^{2}}{\partial x^{2}}+\frac{\partial^{2}}{\partial y^{2}}$ (operador laplaciano).

Assim, por exemplo, se $f: U \longrightarrow \mathbb{C}$ é holomorfa de classe $C^{2}$ então $f$ é harmônica. Com efeito, se $f(z)=u(x, y)+i v(x, y), u=\Re(f), v=\Im(f)$, das identidades de Cauchy-Riemann, obtemos

$$
\begin{gathered}
\frac{\partial^{2} u}{\partial x^{2}}=\frac{\partial^{2} v}{\partial x \partial y}=\frac{\partial}{\partial y} \frac{\partial v}{\partial x}=-\frac{\partial^{2} u}{\partial y^{2}} e \\
\frac{\partial^{2} v}{\partial x^{2}}=-\frac{\partial^{2} u}{\partial x \partial y}=-\frac{\partial}{\partial y} \frac{\partial u}{\partial x}=-\frac{\partial^{2} v}{\partial y^{2}}
\end{gathered}
$$

Logo

$$
\frac{\partial^{2} f}{\partial x^{2}}+\frac{\partial^{2} f}{\partial y^{2}}=\frac{\partial^{2} u}{\partial x^{2}}+\frac{\partial^{2} u}{\partial y^{2}}+i\left(\frac{\partial^{2} v}{\partial x^{2}}+\frac{\partial^{2} v}{\partial y^{2}}\right)=0
$$

Observe que do raciocínio acima, conclui-se também que as partes real e imaginária de $f$ são funções harmônicas.

Além disso, dizemos que $f: U \longrightarrow \mathbb{C}, U$ aberto é uma função subharmônica se

(i) $-\infty \leq u(z)<\infty, \forall z \in U$;

(ii) $\{z \in U:|f(z)|<\alpha\}$ é aberto em $U$ para todo $\alpha$ real;

(iii)Se $\overline{\mathbb{D}}(a, r) \subset U$, onde $\mathbb{D}(a, r)$ é o disco de centro em $a$ e raio $r$, então

$$
u(a) \leq \frac{1}{2 \pi} \int_{-\pi}^{\pi} u\left(a+r e^{i \theta}\right) d \theta \neq-\infty .
$$




\section{Noções Básicas de Probabilidade}

The theory of probability as mathematical discipline can and should be developed from axioms in exactly the same way as Geometry and Algebra.

Andrey Nikolaevich Kolmogorov (1903-1987)

Neste capítulo vamos desenvolver algumas das ferramentas probabilísticas necessárias para as aplicações à Análise Complexa. Neste capítulo e nos dois seguintes seguiremos de perto [bass][2] e [durrett1][6], que são ótimas referências para este assunto.

\subsection{Preliminares}

Vamos inicialmente apresentar alguns conceitos e resultados importantes para o desenvolvimento posterior.

Dados um espaço $\Omega$ e uma $\sigma$-álgebra $\mathcal{F}$ sobre ele, uma medida de probabilidade é medida positiva finita, com medida total 1 , a terna $(\Omega, \mathcal{F}, \mathbb{P})$ é chamada de espaço de probabilidade. Os elementos de $\mathcal{F}$ são chamados de eventos. Funções mensuráveis de $\Omega$ sobre $\mathbb{R}$ são chamadas de variáveis aleatórias e são usualmente denotadas por $X$ ao invés de $f$. A integral de $X$ em relação a $\mathbb{P}$ é chamada de esperança ou valor esperado de $X$ e $\int X(\omega) \mathbb{P}(d \omega)$ é normalmente escrito como $\mathbb{E} X$, enquanto $\int_{A} X(\omega) \mathbb{P}(d \omega)$ será denotado por $\mathbb{E}[X ; A]$. Se um evento ocorre com probabilidade um, dizemos que evento ocorre "quase sempre"e indicamos como q.s. .

Se $A_{n}$ é uma sequência de eventos, definimos $\left(A_{n} i . v\right.$. $)=\cap_{j=1}^{\infty} \cup_{n=j}^{\infty} A_{n}$, onde i.v. significa infinitas vezes.

A seguir alguns resultados bastante úteis: 
(3.1) Teorema da Convergência Monótona: $S e\left(X_{n}\right)_{n}$ é uma sequência monótona crescente de variáveis aleatórias $X_{n}: \Omega \longrightarrow \mathbb{R}_{+}$que converge para $X$ então

$$
\int X d \mathbb{P}=\lim _{n \longrightarrow \infty} \int X_{n} d \mathbb{P}
$$

(3.2)Lema de Fatou: $S e\left(X_{n}\right)_{n}$ é uma sequência de variáveis aleatórias não negativas

$$
\int \liminf _{n \longrightarrow \infty} X_{n} d \mathbb{P} \leq \liminf _{n \longrightarrow \infty} \int X_{n} d \mathbb{P}
$$

(3.3) Teorema da Convergência Dominada: Seja $\left(X_{n}\right)_{n}$ é uma sequência de variáveis aleatórias que converge q.s. para uma variável aleatória $X$. Se existe $Y$ integrável tal que $\left|X_{n}\right| \leq Y$ para todo $n$ então $X$ é integrável $e$

$$
\lim _{n \longrightarrow \infty} \int X_{n} d \mathbb{P}=\int X d \mathbb{P}
$$

(3.4) Lema de Borel-Cantelli(primeira parte): $\quad S e \sum_{n=1}^{\infty} \mathbb{P}\left(A_{n}\right)<\infty$, então $\mathbb{P}\left(A_{n} i . v.\right)=0$.

Demonstração: $\quad$ Note que $\mathbb{P}\left(A_{n}\right.$ i.v. $)=\lim _{j \longrightarrow \infty} \mathbb{P}\left(\cup_{n=j}^{\infty} A_{n}\right)$.

Se $\sum_{n=1}^{\infty} \mathbb{P}\left(A_{n}\right)<\infty$, então $\mathbb{P}\left(\cup_{n=j}^{\infty} A_{n}\right) \leq \sum_{n=1}^{\infty} \mathbb{P}\left(A_{n}\right) \longrightarrow 0$ quando $j \longrightarrow$ $\infty$. 
(3.5) Desigualdade de Markov: Se $X \geq 0$ q.s., então

$$
\mathbb{P}(X \geq a) \leq \mathbb{E} X / a
$$

Demonstração: Segue de $\mathbb{P}(X \geq a)=\mathbb{E}\left[1_{X \geq a}\right] \leq \mathbb{E}[X / a ; X \geq a] \leq$ $\mathbb{E} X / a$.

(3.6) Desigualdade de Jensen: Se g é uma função convexa e $X$ e $g(X)$ são integráveis, então

$$
\mathbb{E}[g(X)] \geq g(\mathbb{E} X)
$$

Demonstração: Se $g$ é convexa, então $g$ passa por cima de suas retas tangentes. Assim, para cada $x_{0}$, existe $c$ tal que $g(X) \leq g\left(x_{0}\right)+c\left(X-x_{0}\right)$.

Fazendo $x_{0}=\mathbb{E}[X]$ e tomando esperanças em ambos os lados obtemos a desigualdade.

A lei ou distribuição de $X$ é a medida de probabilidade $\mathbb{P}_{X}$ sobre $\mathbb{R}$, dada por

$$
\mathbb{P}_{X}(A)=\mathbb{P}(X \in A) .
$$

Dada qualquer medida de probabilidade $\mu$ sobre $\mathbb{R}$, podemos construir uma variável aleatória $X$ tal que $\mathbb{P}_{X}=\mu$. Seja $\Omega=[0,1]$ e seja $\mathbb{P}$ a medida de Lebesgue sobre $[0,1]$. Para $\omega \in[0,1]$, seja $X(\omega)=\inf (t: \mu((-\infty, t]) \geq \omega)$.

Então $\mathbb{P}_{X}((-\infty, a])=\mathbb{P}(X \leq a)=\mu(-\infty, a]$ para cada $a$, assim $\mathbb{P}_{X}=\mu$. 
(3.7) Proposição: Se $f \geq 0$ ou $f$ é limitada,

$$
\mathbb{E}[f(X)]=\int f(X) \mathbb{P}_{X}(d x) .
$$

Demonstração: Se $f=1_{(-\infty, a]}$, então

$$
\mathbb{E}[f(X)]=\mathbb{P}(X \leq a)=\mathbb{P}_{X}((-\infty, a])=\int f(x) \mathbb{P}_{X}(d x) .
$$

Por linearidade obtemos o resultado para funções simples $f$. Tomando limites então temos o resultado para $f$ limitada ou $f$ não negativa.

Um vetor aleatório é função mensurável de $\Omega$ em $\mathbb{R}^{d}$, e a definição de uma distribuição e a proposição anterior se estendem naturalmente neste caso.

(3.8) Proposição: $S e X \geq 0$,

$$
\mathbb{E}\left[X^{p}\right]=\int_{0}^{\infty} p \lambda^{p-1} \mathbb{P}(X>\lambda) d \lambda .
$$

Demonstração: Pelo teorema de Fubini, o lado direito é igual a

$$
\mathbb{E}\left[\int_{0}^{\infty} p \lambda^{p-1} 1_{(X>\lambda)} d \lambda\right]=\mathbb{E}\left[\int_{0}^{X} p \lambda^{p-1} d \lambda\right],
$$

o qual é igual ao lado esquerdo. 


\subsubsection{Independência}

Dois eventos $A$ e $B$ são independentes se

$$
\mathbb{P}(A \cap B)=\mathbb{P}(A) \mathbb{P}(B) .
$$

Esta definição generaliza-se para $n$ eventos: $A_{1}, \ldots, A_{n}$ são independentes se $\mathbb{P}\left(\cap_{i=1}^{j} A_{k_{i}}\right)=\prod_{i=1}^{j} \mathbb{P}\left(A_{k_{i}}\right), 2 \leq j \leq n, 1 \leq k_{1}<k_{2}<\ldots<k_{j} \leq n$. Uma $\sigma$ álgebra $\mathcal{F}$ é independente de uma $\sigma$-álgebra $\mathcal{G}$ se cada $A \in \mathcal{F}$ é independente de cada $B \in \mathcal{G}$. A $\sigma$-álgebra gerada por $X$, denotada por $\sigma(X)$, é a coleção $\{(X \in A) ; A$ boreliano $\}$. Duas variáveis aleatórias são independentes se as $\sigma$-álgebras geradas por $X, Y$ são independentes.

(3.9) Proposição: Se $X, Y$, e $X Y$ são integráveis e $X$ e $Y$ são independentes, então $\mathbb{E}[A B]=\mathbb{E}[A] \mathbb{E}[B]$.

Demonstração: Se $X$ é da forma $\sum_{i=1}^{I} a_{i} 1_{A_{i}}, Y$ é da forma $\sum_{j=1}^{J} b_{j} 1_{B_{i}}$ e $X$ e $Y$ são independentes, então por linearidade e a definição de independência, $\mathbb{E}[A B]=\mathbb{E}[A] \mathbb{E}[B]$. Se $X$ e $Y$ são não negativas podemos aproximá-las por variáveis aleatórias desta forma e aplicar o teorema de convergência monótona o que garante o resultado. O caso geral segue por linearidade.

A função característica de uma variável aleatória $X$ é a transformada de Fourier de sua lei: $\int e^{i u x} \mathbb{P}_{X}(d x)=\mathbb{E} e^{i u X}$ (pela proposição (3.7)). Se $X$ e $Y$ são independentes, então $e^{i u X}$ e $e^{i v Y}$ são também, pela proposição anterior, $\mathbb{E} e^{i(u X+v Y)}=\mathbb{E} e^{i u X} \mathbb{E} e^{i v Y}$. Assim quando $X$ e $Y$ são independentes, a função característica de uma combinação linear de $X$ e $Y$ é o produto das funções características dos fatores.

(3.10) Proposição: $S e \mathbb{E} e^{i(u X+v Y)}=\mathbb{E} e^{i u X} \mathbb{E} e^{i v Y}$ para todos u e v, então $X$ e $Y$ são variáveis aleatórias independentes. 
Demonstração: Sejam $X^{\prime}$ uma variável aleatória com a mesma lei que $X$, $Y^{\prime}$ uma variável aleatória com a mesma lei que $Y$, com $X^{\prime}$ e $Y^{\prime}$ independentes. Então

$$
\mathbb{E} e^{i\left(u X^{\prime}+v Y^{\prime}\right)}=\mathbb{E} e^{i u X^{\prime}} \mathbb{E} e^{i v Y^{\prime}} .
$$

Como $X$ e $X^{\prime}$ têm a mesma lei, elas têm a mesma função característica, e analogamente para $Y$ e $Y^{\prime}$. Portanto $\left(X^{\prime}, Y^{\prime}\right)$ têm a mesma função característica conjunta que $(X, Y)$. Pela unicidade da transformada de Fourier, $\left(X^{\prime}, Y^{\prime}\right)$ tem a mesma lei conjunta que $(X, Y)$, o que implica que $X$ e $Y$ são independentes.

(3.11) Lema de Borel-Cantelli(segunda parte): Se $A_{n}$ é uma sequência de eventos independentes e $\sum_{n=1}^{\infty} \mathbb{P}\left(A_{n}\right)=\infty$, então $\mathbb{P}\left(A_{n}\right)=1$ para infinitos $n$.

Demonstração: Note que

$$
\begin{gathered}
\mathbb{P}\left(\cup_{n=j}^{N} A_{n}\right)=1-\mathbb{P}\left(\cap_{n=j}^{N} A_{n}^{c}\right)=1-\prod_{n=j}^{N}\left(1-\mathbb{P}\left(A_{n}\right)\right) \\
\geq 1-\exp \left(-\sum_{n=j}^{N} \mathbb{P}\left(A_{n}\right)\right) \longrightarrow 1
\end{gathered}
$$

quando $N \longrightarrow \infty$. Assim

$$
\mathbb{P}\left(A_{n} \text { i.v. }\right)=\lim _{j \longrightarrow \infty}\left(\cup_{n=j}^{\infty} A_{n}\right)=1 .
$$




\subsubsection{Variáveis Aleatórias Gaussianas}

Uma variável aleatória normal ou gaussiana de média zero e variância um é uma onde

$$
\mathbb{P}(X \in A)=\int_{A} \frac{1}{\sqrt{2 \pi}} e^{-x^{2} / 2} d x
$$

$A$ boreliano.

Nós também denotamos $X$ como tendo uma lei de distribuição $\mathcal{N}(0,1)$ ("normal 0,1 ", de fato, $X$ tem esperança 0 e variância 1 ).

Uma sequência de variáveis aleatórias $X_{1}, \ldots, X_{n}$ é conjuntamente normal se existe uma sequência variáveis aleatórias independentes $\mathcal{N}(0,1), Z_{1}, \ldots, Z_{n}$ e constantes $b_{i j}$ e $a_{i}$ tais que $X_{i}=\sum_{j=1}^{m} b_{i j} Z_{j}+a_{i}, i=1, \ldots, n$. Em notação matricial, $X=B Z+A$.

Por simplicidade, vamos tomar $A=0$, o que equivale a analisar uma nova variável $X-A$.

A covariância entre duas variáveis aleatórias $X$ e $Y$ é definida como

$$
\operatorname{Cov}(X, Y)=\mathbb{E}[(X-\mathbb{E} X)(Y-\mathbb{E} Y)]
$$

Como estamos assumindo que nossas variáveis aleatórias normais têm média zero, podemos omitir o centro nas esperanças. Dada uma sequência de variáveis aleatórias com média zero, a matriz de covariâncias é dada por $\operatorname{Cov}(X)=\mathbb{E}\left[X X^{t}\right]$, onde $X^{t}$ denota a transposto do vetor $X$. No caso em análise temos

$$
\operatorname{Cov}(X)=\mathbb{E}\left[(B Z)(B Z)^{t}\right]=\mathbb{E}\left[B Z Z^{t} B^{t}\right]=B B^{t}
$$

Vamos calcular a função característica conjunta $\mathbb{E} e^{i u^{t} X}$ do vetor $X$, onde $u$ é um vetor $n$-dimensional. Inicialmente observe que, se $v$ é um vetor $m$ dimensional,

$$
\mathbb{E} e^{i v^{t} Z}=\mathbb{E} \prod_{j=1}^{m} e^{i v_{j} Z_{j}}=\prod_{j=1}^{m} \mathbb{E} e^{i v_{j} Z_{j}}=\prod_{j=1}^{m} e^{-v_{j}^{2}}=e^{-v^{t} v / 2}
$$


pela independência dos $Z \mathrm{~s}$. Assim

$$
\mathbb{E} e^{i u^{t} X}=\mathbb{E} e^{i u^{t} B Z}=e^{-u^{t} B B^{t} u / 2} .
$$

Tomando $u=(0, \ldots, 0, a, 0, \ldots 0)$ como o produto de uma constante pelo vetor unitário na direção da $j$-ésima coordenada, obtemos que cada $X s$ é também normal.

Se $X_{n}$ são variáveis aleatórias normais convergindo em probabilidade (ie, em medida) para uma variável aleatória $X$, então $X$ é também normal. Isto segue de $\mathbb{E} e^{i u X_{n}} \longrightarrow \mathbb{E} e^{i u X}$ por convergência dominada, para vetores aleatórios é análogo.

(3.12) Proposição: Se $Z$ é uma normal $\mathcal{N}(0,1)$ e a $>1$,

$$
\mathbb{P}(Z \geq a) \leq e^{-a^{2} / 2}
$$

Demonstração: O resultado segue de

$$
\mathbb{P}(Z \geq a) \leq \int_{a}^{\infty} e^{-x^{2} / 2} d x \leq \int_{a}^{\infty}(x / a) e^{-x^{2} / 2} d x .
$$

(3.13) Proposição: (caracterização de distribuição multivariada) Um vetor aleatório $v=\left(v_{1}, \ldots, v_{n}\right)$ tem distribuição multivariada gaussiana se e somente se toda combinação linear $\alpha_{1} . v_{1}+\ldots+\alpha_{n} . v_{n}$ é uma gaussiana univariada.

Para uma demonstração deste fato, veja [durrett][7]. 


\subsubsection{Esperança Condicional}

(3.14) Definição: Se $\mathcal{F} \subseteq \mathcal{G}$ são duas $\sigma$-álgebras e $X$ é uma variável aleatória mensurável $\mathcal{G}$-integrável, a esperança condicional de $X$ dado $\mathcal{F}$, escrita como $\mathbb{E}[X \mid \mathcal{F}]$ é qualquer variável aleatória $Y, \mathcal{F}$-mensurável, tal que $\mathbb{E}[Y ; A]=\mathbb{E}[X ; A]$ para todo $A \in \mathcal{F}$.

Se $Y_{1}, Y_{2}$ são duas variáveis aleatórias $\mathcal{F}$-mensuráveis com $\mathbb{E}\left[Y_{1} ; A\right]=\mathbb{E}\left[Y_{2} ; A\right]$ para todo $A \in \mathcal{F}$, então $Y_{1}=Y_{2}$ q.s., ou seja a esperança condicional é única a menos de q.s. (quase sempre) equivalência.

No caso em que $X$ é $\mathcal{F}$-mensurável, temos $\mathbb{E}[X \mid \mathcal{F}]=X$. Se $X$ é independente de $\mathcal{F}, \mathbb{E}[X \mid \mathcal{F}]=\mathbb{E} X$. Ambos os casos seguem imediatamente da definição.

Resultados como o teorema da convergência monótona ou da convergência dominada têm versões com esperança condicionada, assim como as desigualdades de Jensen e Markov, por exemplo, temos:

(3.15) Desigualdade de Jensen para esperanças condicionadas: $S e$ $g$ é convexa e $X$ e $g(X)$ são integráveis,

$$
\mathbb{E}[g(X) \mid \mathcal{F}] \geq g(\mathbb{E}[X \mid \mathcal{F}]) q . s . .
$$

(3.16) Proposição: Se $X$ e $X Y$ são integráveis e $Y$ é mensurável com respeito a $\mathcal{F}$, então

$$
\mathbb{E}[X Y \mid \mathcal{F}]=Y(\mathbb{E}[X \mid \mathcal{F}])(1)
$$

Demonstração: Se $A \in \mathcal{F}$, então para todo $B \in \mathcal{F}$,

$$
\mathbb{E}\left[1_{A} \mathbb{E}[X \mid \mathcal{F}] ; B\right]=\mathbb{E}[\mathbb{E}[X \mid \mathcal{F}] ; A \cap B]=\mathbb{E}[X ; A \cap B]=\mathbb{E}\left[1_{A} X ; B\right]
$$

Como $1_{A} \mathbb{E}[X \mid \mathcal{F}]$ é $\mathcal{F}$-mensurável, isto mostra que o resultado é válido quando 
$Y=1_{A}$ e $A \in \mathcal{F}$. Usando linearidade e tomando limites vemos que o resultado é válido quando $Y$ é $\mathcal{F}$-mensurável e $X$ e $X Y$ são integráveis.

(3.17) Proposição: $\quad S e \mathcal{E} \subseteq \mathcal{F} \subseteq \mathcal{G}$, então

$$
\mathbb{E}[\mathbb{E}[X \mid \mathcal{F}] \mid \mathcal{E}]=\mathbb{E}[X \mid \mathcal{E}]=\mathbb{E}[\mathbb{E}[X \mid \mathcal{E}] \mid \mathcal{F}]
$$

Demonstração: $\quad$ lado direito da igualdade vale porque $\mathbb{E}[X \mid \mathcal{E}]$ é $\mathcal{E}$-mensurável, logo $\mathcal{F}$-mensurável. Para mostrar a igualdade à esquerda, seja $A \in \mathcal{E}$. Como $A$ está também em $\mathcal{F}$,

$$
\mathbb{E}[\mathbb{E}[\mathbb{E}[X \mid \mathcal{F}] \mid \mathcal{E}] ; A]=\mathbb{E}[\mathbb{E}[X \mid \mathcal{F}] ; A]=\mathbb{E}[X ; A]=\mathbb{E}[\mathbb{E}[X \mid \mathcal{E}] ; A]
$$

Como ambos os lados são $\mathcal{E}$-mensuráveis, segue a igualdade.

A existência de $\mathbb{E}[X \mid \mathcal{F}]$ é garantida pela proposição seguinte, cuja prova depende do uso da derivada de Radon-Nikodym (veja bass[2], página 7).

(3.18) Proposição: Se $X$ é integrável, então $\mathbb{E}[X \mid \mathcal{F}]$ existe.

A equação (1) na proposição (3.16), mostra que se $Y$ é $\mathcal{F}$-mensurável, então

$$
\mathbb{E}[Y(X-\mathbb{E}[X \mid \mathcal{F}])]=\mathbb{E}[X Y]-\mathbb{E}[Y \mathbb{E}[X \mid \mathcal{F}]]=0
$$

Assim, um modo de visualizar a esperança condicional é que se $X \in L^{2}(\mathbb{P})$, então $\mathbb{E}[X \mid \mathcal{F}]$ é a projeção de $X$ sobre o subespaço de $L^{2}(\mathbb{P})$ gerado pelas funções $\mathcal{F}$-mensuráveis, ou seja, o operador esperança condicional sobre $L^{2}(\mathbb{P})$ é o operador projeção sobre este subespaço. O operador esperança condicional sobre $L^{1}(\mathbb{P})$ é a (única) extensão deste operador projeção. 


\section{Noções Básicas de Processos Estocásticos}

Le calcul des probabilités n'est au fond que le bon sens réduit au calcul.

Pierre Simon Laplace (1749-1827)

\subsection{Preliminares}

Neste capítulo continuamos a tratar das ferramentas necessárias para o nosso trabalho, agora estudando processos estocásticos, em particular o movimento browniano, deixando de lado tratamentos mais gerais.

Seja $(\Omega, \mathcal{F}, \mathbb{P})$ um espaço de probabilidades e seja $\mathcal{B}$ a $\sigma$-álgebra de Borel sobre $[0, \infty)$. Uma filtração $\left(\mathcal{F}_{t}\right)_{t} \subset \mathcal{F}$ em $(\Omega, \mathcal{F}, \mathbb{P})$ é uma família crescente de $\sigma$-álgebras.

Um processo estocástico (em tempo contínuo), denotado por $X(t, \omega)$ ou $X_{t}(\omega)$ ou ainda por $X_{t}$ é uma função de $[0, \infty) \times \Omega$ em $\mathbb{R}^{d}$ que é mensurável com respeito a $\sigma$-álgebra produto de $\mathcal{B}$ e $\mathcal{F}$.

Um processo $X_{t}, 0 \leq t<\infty$, é chamado processo gaussiano se o vetor $\left(X_{t_{1}}, X_{t_{2}}, \ldots, X_{t_{n}}\right), 0 \leq t_{1}<t_{2}<\ldots<t_{n}$ tem uma distribuição gaussiana multivariada para toda sequência finita.

Um processo parado é um processo estocástico que mantém seu estado constante após um dado tempo (este tempo pode ser aleatório), já um processo morto é um processo estocástico que assume um estado "indefinido" após um dado tempo, (estaremos interessados no caso em o processo deixa algum conjunto $\mathrm{A}$, assim dizemos que o estado assumido passa a estar num "cemitério") o chamado instante de morte ou "killing time" (este tempo também pode ser aleatório). 


\subsection{Tempos de Parada}

Seja $X_{t}$ um processo estocástico e uma filtração $\mathcal{F}_{t}$. Suponha que cada $\mathcal{F}_{t}$ é contínua à direita, ie, $\mathcal{F}_{t}=\mathcal{F}_{t+}$ para cada $t$, onde $\mathcal{F}_{t+}=\cap_{\epsilon>0} \mathcal{F}_{t+\epsilon}$.

Suponha também que cada $X_{t}$ é adaptado a $\mathcal{F}_{t}$ : para cada $t, X_{t}$ é $\mathcal{F}_{t^{-}}$ mensurável.

(4.1) Definição: Uma variável aleatória $T$ de $\Omega$ em $[0, \infty)$ é chamada um tempo de parada ("stopping time") se para cada $t,(T<t)=\{\omega$ : $T(\omega)<t\} \in \mathcal{F}_{t}$ para todo $t \in[0, \infty)$.

A intuição por trás da idéia de tempo de parada é que o processo "sabe"se $T$ ocorreu no instante $t$ olhando através de $\mathcal{F}_{t}$. Suponha que dois ciclistas se dirigem na direção sul, através de uma ciclovia e devem parar na primeira lanchonete após o quilômetro trinta. Este é um exemplo de tempo de parada, no entanto, se os ciclistas tivessem que parar na penúltima lanchonete antes do quilômetro trinta (que não teria sido alcançado ainda), este não seria um exemplo de tempo de parada.

Vamos agora ver algumas maneiras de obter novos tempos de parada a partir de outros:

(4.2) Proposição: Temos que:

(i) $T$ é um tempo de parada se e somente se $(T \leq t) \in \mathcal{F}_{t}$ para todo $t$;

(ii) Tempos fixos t são tempos de parada;

(iii) Se $S$ e $T$ são tempos de parada, então $S \wedge T$ e $S \vee T$ são tempos de parada, onde $a \wedge b=\min \{a, b\}$ e $a \vee b=\max \{a, b\}$;

(iv) Se $T_{n}$ é sequência não decrescente de tempos de parada, então $T=$ $\sup _{n} T_{n}$ é um tempo de parada;

(v) Se $T_{n}$ é sequência não crescente de tempos de parada, então $T=\inf _{n} T_{n}$ é um tempo de parada;

(vi) Se $S$ é um tempo de parada, então $S+t$ é um tempo de parada. 
Demonstração: Todos os resultados seguem diretamente da definição. Vejamos o primeiro:

(i) se $T$ é um tempo de parada, então

$$
(T \leq t)=\cap_{n>N}(T<t+1 / n) \in \cap_{n>N} \mathcal{F}_{t+1 / n}
$$

para cada $N$. Assim $(T \leq t) \in \mathcal{F}_{t+}=\mathcal{F}_{t}$. Por outro lado, se $(T \leq t) \in \mathcal{F}_{t}$, para todo $t$, então $(T<t)=\cup_{n=1}^{\infty}(T \leq t-1 / n) \in \mathcal{F}_{t}$, pois $\mathcal{F}_{t}$ é crescente.

Entre os tipos de tempos de parada em que estamos interessados estão os tempos em que $X_{t}$ atinge um conjunto $A$ ou deixa um conjunto $A$ (o segundo caso é equivalente ao primeiro, considerando atingir $A^{c}$ ).

No caso em que estamos interessados em que $X_{t}$ atinge ou alcança um conjunto $A$, denotamos $T_{A}=\inf \left\{t>0: X_{t} \in A\right\}$.

No caso em que estamos interessados em que $X_{t}$ deixa ou sai de um conjunto $A$, denotamos $\tau_{A}=\inf \left\{t>0: X_{t} \notin A\right\}$.

Naturalmente, $T_{A}=\tau_{A}^{c}$ e $\tau_{A}=T_{A}^{c}$.

(4.3) Proposição: Temos que:

(i) Se A é um conjunto aberto, $T_{A}$ é um tempo de parada;

(ii) Se A é um conjunto fechado, $T_{A}$ é um tempo de parada.

Demonstração: Suponha que $A$ é aberto. Se $T<t$, então para algum $s \geq t$, nós temos $X_{s} \in A$. Como as trajetórias são contínuas, existe um racional $q<t \operatorname{com} X_{q} \in A$. Assim,

$$
(T<t)=\cup_{q<t, q \in \mathbb{Q}}\left(X_{q} \in A\right) \in \mathcal{F}_{t},
$$

o que prova (i).

Se $A$ é fechado, seja $A_{n}=\{x: \operatorname{dist}(x, A)<1 / n\}$, onde $\operatorname{dist}(x, A)$ denota a distância entre $x$ e $A$. Os conjuntos $A_{n} s$ são abertos, $\operatorname{logo} T=A_{n}$ 
é uma sequência crescente de tempos de parada, pela proposição anterior, $T=\sup _{n} T_{A_{n}}$ é um tempo de parada. Como $T_{A} \geq T_{A_{n}}$ para cada $n$, então $T_{A} \geq T$. Se $T=\infty$, então $T_{A}$ deve ser igual a $\infty$. Se $T<\infty$, pela continuidade das trajetórias, $X_{T}=\lim X_{T_{A_{n}}}$. Como $X_{T_{A_{n}}}$ está no fecho de $A_{m}$ se $n \geq m$, segue que $X_{T}$ está também para cada $m$. Isto implica que $X_{T} \in A$, ou $T_{A} \leq T$, portanto $T_{A}=T$, o que prova (ii).

\subsection{Propriedade de Markov}

\subsubsection{Definições}

Seja $\mathcal{F}_{s}^{00}=\sigma\left(X_{r} ; r \leq s\right), s \in[0, \infty]$. Esta $\sigma$-álgebra é chamada a "história" do processo até o instante $s$ inclusive.

Um processo estocástico $X(t)$ é chamado um markoviano se

$$
\mathbb{P}\left(X(t) \in A \mid \mathcal{F}_{s}^{00}\right)=\mathbb{P}(X(t) \in A \mid \sigma(X(s)))
$$

q.s. para todo $0 \leq s \leq t$ e $A$ boreliano.

A idéia por trás desta definição é que, dado apenas o valor atual $X(s)$, podese predizer valores esperados futuros de $X(t)$ tão bem quanto se conhecesse toda a história do processo antes do instante $s$. Em outras palavras, o processo apenas "sabe" seu valor no instante $s$ e não "lembra" como era.

\subsection{Martingais}

\subsubsection{Definições}

Nesta seção vamos tratar com martingais tanto em tempo discreto quanto em tempo contínuo. Seja $\mathcal{F}_{n}$ uma sequência crescente de $\sigma$-álgebras. Uma sequência de variáveis aleatórias $M_{n}$ é adaptada a $\mathcal{F}_{n}$ se para cada $n$ inteiro, $M_{n}$ é $\mathcal{F}_{n}$-mensurável. Similarmente uma coleção de variáveis aleatórias $M_{t}$ é adaptada a $\mathcal{F}_{t}$ se para cada $t$ real, $M_{t}$ é $\mathcal{F}_{t}$-mensurável. 
Nós dizemos que a filtração $\mathcal{F}_{t}$ satisfaz as condições usuais se ela é contínua à direita (ie, $\mathcal{F}_{t}=\mathcal{F}_{t+}$ para todo $t$, onde $\mathcal{F}_{t+}=\cap_{\epsilon>0} \mathcal{F}_{t+\epsilon}$ ) e cada $\mathcal{F}_{t}$ é $\mathbf{c o m}$ pleto (ie, $\mathcal{F}_{t}$ contém todos os conjuntos de medida nula, segundo $\mathbb{P}$ ). Nesta seção haverá apenas uma medida de probabilidade e assumiremos que a filtração $\mathcal{F}_{t}$ satisfaz as condições usuais.

(4.4) Definições: $M_{n}$ é um martingal se $M_{n}$ é adaptado a $\mathcal{F}_{n}, M_{n}$ é integrável para todo $n$, e $\mathbb{E}\left[M_{n} \mid \mathcal{F}_{n-1}\right]=M_{n-1}$, q.s., para $n=2,3, \ldots$. Similarmente, $M_{t}$ é um martingal se $M_{t}$ é integrável para todo $t, \mathcal{F}_{t}$-adaptada, e $\mathbb{E}\left[M_{t} \mid \mathcal{F}_{s}\right]=M_{s}$, q.s., se $s \leq t$.

Se nós temos $\mathbb{E}\left[M_{t} \mid \mathcal{F}_{s}\right] \geq M_{s}$, q.s., para todo $s \leq t$, então $M_{t}$ é um submartingal. Se nós temos $\mathbb{E}\left[M_{t} \mid \mathcal{F}_{s}\right] \leq M_{s}$, q.s., para todo $s \leq t$, então $M_{t}$ é um supermartingal.

\subsubsection{Parada Opcional}

Note que para martingais discretos temos por indução que $\mathbb{E}\left[M_{n}\right]=\mathbb{E}\left[M_{0}\right]$. O teorema que está na base de todos os resultados sobre martingais é teorema de parada opcional de Doob, o qual diz que o mesmo resultado vale se trocarmos $n$ por um tempo de parada $N$. Existem várias versões dependendo das condições que colocamos nos tempos de parada.

(4.5) Teorema(para martingais em tempo discreto): Se $N$ é tempo de parada limitado com respeito a $\mathcal{F}_{n}$ e $M_{n}$ um martingal, então $\mathbb{E} M_{N}=$ $\mathbb{E} M_{0}$.

Demonstração: Como $N$ é limitada, façamos $K$ igual ao maior valor que $N$ assume. Assim,

$$
\mathbb{E} M_{N}=\sum_{k=0}^{K} \mathbb{E}\left[M_{N} ; N=k\right]=\sum_{k=0}^{K} \mathbb{E}\left[M_{k} ; N=k\right]
$$

Note que $(N=k)$ é $\mathcal{F}_{j}$-mensurável se $j \geq k$, então $\mathbb{E}\left[M_{k} ; N=k\right]=$ 
$\mathbb{E}\left[M_{k+i} ; N=k\right]$ para todo $1 \leq i \leq K-k$.

Assim,

$$
\mathbb{E} M_{N}=\sum_{k=0}^{K} \mathbb{E}\left[M_{K} ; N=k\right]=\mathbb{E} M_{K}=\mathbb{E} M_{0}
$$

(4.6) Corolário: $S e N$ é limitado por $K$ e $M_{n}$ é um submartingal, então $\mathbb{E} M_{N} \leq \mathbb{E} M_{K}$.

As dificuldades vêm quando consideramos tempos de parada não limitados ou em tempo contínuo. Note que uma coleção de variáveis aleatórias é uniformemente integrável se

$$
\mathbb{E}\left[\left|X_{n}\right| ;\left|X_{n}\right| \geq a\right] \longrightarrow 0
$$

quando $a \longrightarrow \infty$, uniformemente em $n$.

Anteriormente vimos uma versão em esperança condicional da desigualdade de Jensen. Se $X_{t}$ é um martingal ou submartingal não-negativo, $g$ é convexa, e $g\left(\left|X_{t}\right|\right)$ é integrável, então

$$
\mathbb{E}\left[g\left(\left|X_{t}\right|\right) \mid \mathcal{F}_{s}\right] \geq g\left(\left|\mathbb{E}\left[X_{t} \mid \mathcal{F}_{s}\right]\right|\right)
$$

(4.7) Teorema(para martingais em tempo contínuo): $S e M_{t}$ é um martingal contínuo à direita e $T$ é um tempo de parada limitado por $K$, então

$$
\mathbb{E}\left[M_{T}\right]=\mathbb{E}\left[M_{K}\right]=\mathbb{E}\left[M_{0}\right]
$$

Se $M$ é um submartingal não negativo, nós temos $\mathbb{E}\left[M_{T}\right] \leq \mathbb{E}\left[M_{K}\right]$. 
Demonstração: Seja a sequência de tempos de parada $T_{n}$ dada por

$T_{n}(\omega)=(k+1) K / 2^{n}$ se $k K / 2^{n} \leq T(\omega)<(k+1) K / 2^{n}$.

$M_{k K / 2^{n}}$ é um martingal em tempo discreto com respeito a $\mathcal{F}_{k K / 2^{n}}$ assim, temos pelo teorema (4.5) que $\mathbb{E}\left[M_{T_{n}}\right]=\mathbb{E}\left[M_{K}\right]$ para cada $n$. Como $M_{K}$ é integrável, existe uma função convexa crescente não negativa $g \operatorname{com} g(x) / x \longrightarrow$ $\infty$ quando $x \longrightarrow \infty$ tal que $\mathbb{E}\left[g\left(\left|M_{K}\right|\right)\right]<\infty$. Como $g\left(\left|M_{t}\right|\right)$ é um submartingal, $\mathbb{E}\left[g\left(\left|M_{T_{n}}\right|\right)\right] \leq \mathbb{E}\left[g\left(\left|M_{K}\right|\right)\right]<\infty$ e assim as variáveis aleatórias $\left|M_{T_{n}}\right|$ são uniformemente integráveis. Fazendo $n \longrightarrow \infty, T_{n} \downarrow T$, e pela continuidade à direita, concluímos que $M_{T_{n}} \longrightarrow M_{T}$ e assim $\mathbb{E}\left[M_{T}\right]=\mathbb{E}\left[M_{K}\right]$. O outro caso é análogo.

(4.8) Corolário: $S e S \leq T$ são tempos de parada limitados por $K$ e $M$ é um martingal contínuo à direita, então $\mathbb{E}\left[M_{T} \mid \mathcal{F}_{S}\right]=M_{S}$, q.s. .

Demonstração: Suponha que $A \in \mathcal{F}_{S}$. Precisamos mostrar que $\mathbb{E}\left[M_{S} ; A\right]=\mathbb{E}\left[M_{T} ; A\right]$. Defina um novo tempo de parada $U$ por $U(\omega)=S(\omega)$ se $\omega \in A$ e $U(\omega)=T(\omega)$ se $\omega \notin A$.

De fato, $U$ é um tempo de parada, pois

$$
\mathbb{E}\left[M_{U}\right]=\mathbb{E}\left[M_{K}\right]=\mathbb{E}\left[M_{T}\right]
$$

o que implica

$$
\mathbb{E}\left[M_{S} ; A\right]+\mathbb{E}\left[M_{T} ; A^{C}\right]=\mathbb{E}\left[M_{T}\right]
$$

Agora, basta subtrair $\mathbb{E}\left[M_{T} ; A^{C}\right]$ de cada lado para completar a demonstração.

Note que se $S$ e $T$ são tempos de parada limitados, então

$$
\mathbb{E}\left[\left(M_{T}-M_{S}\right)^{2} \mid \mathcal{F}_{S}\right]=\mathbb{E}\left[M_{T}^{2} \mid \mathcal{F}_{S}\right]-2 M_{S} \mathbb{E}\left[M_{T} \mid \mathcal{F}_{S}\right]+M_{S}^{2}=\mathbb{E}\left[M_{T}^{2}-M_{S}^{2} \mid \mathcal{F}_{S}\right]
$$


Tomando esperanças, obtemos:

$$
\mathbb{E}\left[\left(M_{T}-M_{S}\right)^{2}\right]=\mathbb{E}\left[M_{T}^{2}\right]-\mathbb{E}\left[M_{S}^{2}\right]
$$

\subsubsection{Desigualdades de Doob}

Vamos agora tratar das primeiras aplicações interessantes dos resultados anteriores, as desigualdades de Doob. Se $M_{t}$ ou $M_{n}$ são martingais respectivamente em tempo contínuo e discreto, denote $M_{t}^{*}=\sup _{s \leq t}\left|M_{s}\right|$, e analogamente para $M_{n}^{*}$.

(4.9) Teorema: $S e M_{n}$ é um martingal, $\mathbb{P}\left(M_{n}^{*} \geq a\right) \leq \mathbb{E}\left|M_{n}\right| / a$. O mesmo resultado vale para $M_{t}$ se $M_{t}$ é um martingal ou submartingal positivo com trajetórias contínuas à direita.

Demonstração: Seja $N=\min \left\{j:\left|M_{j}\right| \geq a\right\}$. Como $|$.$| é convexa, \left|M_{n}\right|$ é um submartingal. Como $\left|M_{N}\right| \geq a$ sobre $(N<\infty)$,

$$
\mathbb{P}\left(M_{n}^{*} \geq a\right)=\mathbb{P}(N \leq n) \leq \mathbb{E}\left[\left|M_{N}\right| / a ; N \leq n\right] \leq \mathbb{E}\left|M_{N \wedge n}\right| / a \leq \mathbb{E}\left|M_{n}\right| / a
$$

Para $p>1$, nós temos a seguinte desigualdade:

(4.10) Teorema: $S e p>1$, existe $c$ dependendo somente de $p$ tal que

$$
\mathbb{E}\left[\left(M_{n}^{*}\right)^{p}\right] \leq c \mathbb{E}\left[\left|M_{n}\right|^{p}\right]
$$

com o mesmo sendo verdade se $M_{t}$ é martingal ou submartingal positivo com trajetórias contínuas à direita. 
Demonstração: Temos que $\left|M_{j}\right|$ é submartingal e assim

$$
\left|M_{j}\right| \leq \mathbb{E}\left[\left|M_{n}\right| \mid \mathcal{F}_{j}\right] \leq\|M\|_{\infty}
$$

Se $p=\infty$ a prova é imediata. Agora, sejam $M_{n}^{1}=M_{n} 1_{\left|M_{n}\right|>a / 2}, M_{n}^{2}=$ $M_{n}-M_{n}^{1}$. Seja $M_{j}^{i}=\mathbb{E}\left[M_{n}^{i} \mid \mathcal{F}_{j}\right], j=1,2$. Como $\max _{j}\left|M_{j}\right| \leq \max _{j}\left|M_{j}^{1}\right|+a / 2$, pelo teorema anterior em $M^{1}$ temos

$$
\begin{gathered}
\mathbb{P}\left(M_{n}^{*}>a\right) \leq \mathbb{P}\left(\left(M_{n}^{1}\right)^{*}>a / 2\right) \leq 2 \mathbb{E}\left[\left|M_{n}^{1}\right|\right] / a \\
=2 \mathbb{E}\left[\left|M_{n}\right| ;\left|M_{n}\right|>a / 2\right] / a .
\end{gathered}
$$

Assim, pela proposição (3.8),

$$
\begin{aligned}
& \mathbb{E}\left[\left(M_{n}^{*}\right)^{p}\right]=\int_{0}^{\infty} p a^{p-1} \mathbb{P}\left(M_{n}^{*}>a\right) d a \\
& \leq \int_{0}^{\infty} 2 p a^{p-2} \mathbb{E}\left[\left|M_{n}\right| 1_{\left(\left|M_{n}\right|>a / 2\right)}\right] d a .
\end{aligned}
$$

Pelo teorema de Fubini a última integral é

$$
\mathbb{E}\left[\int_{0}^{2\left|M_{n}\right|} 2 p a^{p-2} d a\left|M_{n}\right|\right]=\mathbb{E}\left[\frac{2^{p} p}{p-1}\left|M_{n}\right|^{p}\right],
$$

como desejado.

\subsubsection{Teoremas de Convergência para Martingais}

Os teoremas de convergência para martingais são um conjunto importante de consequências das paradas opcionais. O passo principal é o lema seguinte (lema "upcrossing"). O número de passagens de baixo para cima ("upcrossings") de um intervalo $[a, b]$ é o número de vezes que um processo cruza 
vindo de "baixo" de $a$ para "cima" de $b$.

Para ser mais exato, sejam $S_{1}=\min \left\{k: X_{k} \geq a\right\}, T_{1}=\min \left\{k>S_{1}: X_{k} \geq\right.$ $b\}$, e $S_{i+1}=\min \left\{k>T_{i}: X_{k} \leq a\right\}, T_{i+1}=\min \left\{k>S_{i+1}: X_{k} \geq b\right\}$.

O número de passagens $U_{n}$ antes do tempo $n$ é $U_{n}=\max \left\{j: T_{j} \leq n\right\}$.

(4.11) Lema: $S e X_{k}$ é um submartingal,

$$
\mathbb{E} U_{n} \leq(b-a)^{-1} \mathbb{E}\left[\left(X_{n}-a\right)^{+}\right]
$$

Demonstração: Primeiro assuma que $a=0$ e $X_{k} \geq 0$ para cada $k$. Fixe $n$ e defina $X_{m}=X_{n}$ para $m \geq n$, este ainda é submartingal. Sejam $S_{i}$ e $T_{i}$ como acima, e sejam $S_{i}^{\prime}=S_{i} \wedge(n+1), T_{i}^{\prime}=T_{i} \wedge(n+1)$.

Escrevendo

$$
\mathbb{E} X_{n+1}=\mathbb{E} X_{S_{1}^{\prime}}+\sum_{i=0}^{\infty} \mathbb{E}\left[X_{T_{i}^{\prime}}-X_{S_{i}^{\prime}}\right]+\sum_{i=0}^{\infty} \mathbb{E}\left[X_{S_{i+1}^{\prime}}-X_{T_{i}^{\prime}}\right]
$$

Todos os somandos no terceiro termo à direita são não negativos, pois $X_{k}$ é um submartingal, e

$$
\sum_{i=0}^{\infty} \mathbb{E}\left[X_{T_{i}^{\prime}}-X_{S_{i}^{\prime}}\right] \geq(b-a) U_{n}
$$

Logo

$$
\mathbb{E} U_{n} \leq \mathbb{E} X_{n+1} / b .(*)
$$

Vamos remover a hipótese de que $a=0$ e $X_{k} \geq 0$. O número de passagens de baixo para cima de $[a, b]$ por $X_{k}$ é o mesmo que o número de passagens de baixo para cima de $[0, b-a]$ por $Y_{k}=\left(X_{k}-a\right)^{+}$. Logo basta aplicar (*) ao número de passagens de baixo para cima de $[0, b-a]$ pelo processo $\left(X_{k}-a\right)^{+}$. 
O que leva ao teorema de convergência para martingais.

(4.12) Teorema: $S e X_{n}$ é um submartingal tal que $\sup _{n} \mathbb{E} X_{n}^{+}<\infty$, então $X_{n}$ converge q.s. quando $n \longrightarrow \infty$.

Demonstração: Seja $U(a, b)=\lim _{n \longrightarrow \infty} U_{n}$. Para cada $a, b$ racionais, por convergência monótona, $\mathbb{E}[U(a, b)] \leq c(b-a)^{-1}<\infty$. Assim $U(a, b)<\infty$, q.s. . Tomando a união sobre todos os pares de racionais $a, b$, nós temos que q.s. a sequência $X_{n}(\omega)$ não poderá ter $\lim \sup X_{n}>\liminf X_{n}$. Logo $X_{n}$ converge q.s., embora ainda exista a possibilidade do limite ser infinito. Como $X_{n}$ é um submartingal, $\mathbb{E} X_{n} \geq \mathbb{E} X_{0}$, assim temos

$$
\mathbb{E}\left|X_{n}\right|=\mathbb{E} X_{n}^{+}+\mathbb{E} X_{n}^{-}=2 \mathbb{E} X_{n}^{+}-\mathbb{E} X_{n} \leq 2 \mathbb{E} X_{n}^{+}-\mathbb{E} X_{0}
$$

Pelo lema de Fatou,

$$
\mathbb{E}\left[\lim _{n}\left|X_{n}\right|\right] \leq \sup _{n} \mathbb{E}\left|X_{n}\right|<\infty
$$

ou $X_{n}$ converge q.s. para um limite finito.

(4.13) Corolário: $S e X_{n}$ é um supermartingal positivo ou um martingal limitado por baixo ou por cima, $X_{n}$ converge q.s..

Demonstração: Se $X_{n}$ é um supermartingal positivo, $-X_{n}$ é um submartingal limitado por cima por zero. Agora, aplicamos o teorema anterior.

Se $X_{n}$ é um martingal limitado por cima, considere $-X_{n}$, podemos assumir que $X_{n}$ é limitado por baixo. Considerar $X_{n}+M$ para um fixo $M$ não irá 
afetar a convergência, assim podemos assumir que $X_{n}$ é limitado por baixo por zero, agora basta aplicar a primeira parte já provada.

(4.14) Corolário: Os dois resultados anteriores, (4.12) e (4.13), permanecem válidos se considerarmos martingais ou supermartingais ou submartingais contínuos com trajetórias contínuas à direita.

Demonstração: A prova que limsup $\left|X_{t}\right|$ é finito é a mesma que na última linha da demonstração do teorema (4.12). Assim, a possibilidade de oscilação, ie, $\lim \sup \left|X_{t}\right|>\liminf \left|X_{t}\right|$ é a única que resta a ser descartada. O mesmo argumento do teorema (4.12) mostra que $\mathbb{E}\left[U_{t}(a, b)\right] \leq \mathbb{E}\left[\left(X_{t}-a\right)^{+}\right]$, onde $U_{t}(a, b)$ é o número de passagens de baixo para cima de $[a, b]$ por $X_{t}$ no tempo $t$, e basta retomar a demonstração do teorema (4.12).

(4.15) Corolário: $S e X_{t}$ é um martingal contínuo à direita com $\sup _{t} \mathbb{E}\left|X_{t}\right|^{p}<\infty$ para algum $p>1$, então a convergência é em $L^{p}$ q.s. .

Este resultado permanece válido quando $X_{t}$ é um submartingal contínuo à direita. Se $X_{t}$ é um martingal uniformemente integrável com trajetórias contínuas à direita, então a convergência é em $L^{1}$. Se $X_{t} \longrightarrow X_{\infty}$ em $L^{1}$, então $X_{t}=\mathbb{E}\left[X_{\infty} \mid \mathcal{F}_{t}\right]$.

OBS.: $X_{t}$ é um martingal uniformemente integrável se a coleção de variáveis aleatórias $X_{t}$ é uniformemente integrável.

Demonstração: A afirmação sobre convergência em $L^{p}$ segue da desigualdade de Doob e por convergência dominada. A afirmação sobre convergência em $L^{1}$ segue, haja visto que convergência q.s. e integrabilidade uniforme implicam convergência em $L^{1}$. Por fim, se $t<n, X_{t}=\mathbb{E}\left[X_{n} \mid \mathcal{F}_{t}\right]$. Se $A \in \mathcal{F}_{t}$,

$$
\mathbb{E}\left[X_{t} ; A\right]=\mathbb{E}\left[X_{n} ; A\right] \longrightarrow \mathbb{E}\left[X_{\infty} ; A\right]
$$


pela convergência em $L^{1}$ de $X_{n}$ para $X_{\infty}$. Como isto é verdade para todo $A \in \mathcal{F}_{t}, X_{t}=\mathbb{E}\left[X_{\infty} \mid \mathcal{F}_{t}\right]$.

Sejam $D_{n}=\left\{k / 2^{n}: k \leq 2^{n}\right\}$ e $D=\cup_{n} D_{n}$, temos ainda o seguinte corolário:

(4.16) Corolário: Suponha que $X_{t}$ é um submartingal com $\sup _{t} \mathbb{E} X_{t}^{+}<$ $\infty$. Então $\left\{X_{t}: t \in D\right\}$ têm limites à direita e à esquerda q.s. .

Demonstração: Seja $U_{n}(a, b)$ o número de passagens de baixo para cima em $[a, b]$ de $X_{t}, t \in D_{n}$, e $U(a, b)$ o número de passagens de baixo para cima em $[a, b]$ de $X_{t}, t \in D$. Por convergência monótona,

$$
\mathbb{E}[U(a, b)]=\lim _{n} \mathbb{E}\left[U_{n}(a, b)\right] \leq \frac{\mathbb{E}\left[\left(X_{1}-a\right)^{+}\right]}{b-a}<\infty .
$$

Isto é verdade para todo par de racionais $a, b$, juntando com o argumento do teorema (4.12) temos a prova desejada.

\subsubsection{Decomposições de Supermartingais}

A decomposição de Doob-Meyer diz que sob hipóteses razoáveis, um supermartingal pode ser decomposto em um martingal menos um processo crescente. Para provar o caso de tempo contínuo e trajetórias contínuas, vamos começar com uma aproximação discreta e então mostrar a convergência em $L^{2}$ das aproximações.

(4.17) Teorema: Suponha que $Z_{t}$ é um supermartingal com trajetórias contínuas. Então existe um martingal $M_{t}$ e um processo crescente $A_{t}$, ambos com trajetórias contínuas e adaptado à filtração de $Z_{t}$, tal que $Z_{t}=M_{t}-A_{t}$. Mais ainda, esta decomposição é única. 
(4.18) Proposição: Suponha que $M_{t}$ é um martingal contínuo com trajetórias de variação limitada e $M_{0} \equiv 0$. Então $M$ é identicamente nulo.

Demonstração: Seja $T_{N}=\inf \left\{t>0:\left|M_{t}\right|>N\right.$ ou $\left.\int_{0}^{t}\left|d M_{s}\right|>N\right\}$ $\left(\int_{0}^{t}\left|d M_{s}\right|\right.$ é a variação limitada de $M_{s}$ até o tempo $\left.t\right)$. Vamos mostrar que $M_{t \wedge T_{N}}$ é identicamente nulo e fazer $N \longrightarrow \infty$, assim, suponha que $M$ é limitado e tem variação total limitada por $N$.

Seja $\epsilon>0$, e sejam $S_{0}=0$ e $S_{i+1}=\inf \left\{t>S_{i}:\left|M_{t}-M_{S_{i}}\right|>\epsilon\right\}$. Por (4.12) temos que

$$
\begin{gathered}
\mathbb{E} M_{\infty}^{2}=\mathbb{E}\left[\sum_{i=0}^{\infty}\left(M_{S_{i+1}}-M_{S_{i}}\right)^{2}\right] \\
\leq \epsilon \mathbb{E}\left[\sum_{i=0}^{\infty} \mathbb{E}\left|M_{S_{i+1}}-M_{S_{i}}\right|\right] \leq \epsilon \mathbb{E}\left[\int_{0}^{\infty}\left|d M_{s}\right|\right] .
\end{gathered}
$$

Como $\epsilon$ é arbitrário, $\mathbb{E} M_{\infty}^{2}=0$. Pela desigualdade de Doob, temos: $\mathbb{E} M_{s}^{2}=0$.

Vamos provar agora a unicidade no teorema (4.17):

Demonstração: Seja $Z_{t}=M_{t}-A_{t}=N_{t}-B_{t}$, onde $M_{t}$ e $N_{t}$ são martingais e $A_{t}$ e $B_{t}$ são processos de variação limitada, então $M_{t}-N_{t}=A_{t}-B_{t}$ é um martingal com trajetórias contínuas que também é de variação limitada. Aplicando a proposição (4.18) em $(M-N)_{t}-(M-N)_{0}$ temos $M_{t}=N_{t}$, $A_{t}=B_{t}$, e assim a unicidade.

Os seguintes lemas são úteis para muitas aplicações além da prova de existência. 
(4.19) Lema: Suponha que $A_{k}$ é um processo de variação limitada com $A_{0} \equiv 0$ e também que $A_{k}$ é $\mathcal{F}_{k-1}$-mensurável e ainda que $\mathbb{E}\left[\left|A_{\infty}-A_{k}\right| \mathcal{F}_{k}\right] \leq$ $N, k=0,1,2, \ldots$. Então

$$
\mathbb{E} A_{\infty}^{2} \leq 2 N^{2}
$$

Demonstração: Seja $A_{k}$ um processo de variação limitada com $A_{0} \equiv 0$. Seja $a_{k}=A_{k+1}-A_{k}$. Depois de algumas manipulações algébricas obtemos:

$$
A_{\infty}^{2}=2 \sum_{k=0}^{\infty}\left(A_{\infty}-A_{k}\right) a_{k}-\sum_{k=0}^{\infty} a_{k}^{2}(1) .
$$

Se $A_{k}$ é também crescente, de modo que $a_{k} \geq 0$ para todo $k$, então

$$
\begin{gathered}
\mathbb{E} A_{\infty}^{2}=2 \mathbb{E}\left[\sum_{k=0}^{\infty} \mathbb{E}\left[A_{\infty}-A_{k} \mid \mathcal{F}_{k}\right] a_{k}\right]-\mathbb{E}\left[\sum_{k=0}^{\infty}\left(a_{k}\right)^{2}\right] \\
\leq 2 N \mathbb{E}\left[\sum_{k=0}^{\infty} a_{k}\right]=2 N \mathbb{E} A_{\infty} .
\end{gathered}
$$

Como $\mathbb{E}\left[A_{\infty}-A_{k} \mid \mathcal{F}_{0}\right] \leq N$ e $A_{0} \equiv 0$, tomando esperanças temos $\mathbb{E} A_{\infty} \leq N$ e substituindo na equação anterior temos o resultado desejado.

(4.20) Lema: Suponha que $A_{k}^{(1)}$ e $A_{k}^{(2)}$ são processos crescentes satisfazendo as hipóteses do lema anterior. Seja $B_{k}=A_{k}^{(1)}-A_{k}^{(2)}$. Suponha que existe $W \geq 0$ com $\mathbb{E} W^{2}<\infty$ tal que para todo $k$,

$$
\left|\mathbb{E}\left[B_{\infty}-B_{k} \mid \mathcal{F}_{k}\right]\right| \leq \mathbb{E}\left[W \mid \mathcal{F}_{k}\right]
$$


Então existe c tal que

$$
\mathbb{E}\left[\sup _{k} B_{k}^{2}\right] \leq c \mathbb{E} W^{2}+c N\left(\mathbb{E} W^{2}\right)^{1 / 2} .
$$

Demonstração: Seja $b_{k}=B_{k+1}-B_{k}$. Seja $a_{k}^{(i)}=A_{k+1}^{(i)}-A_{k}^{(i)}, i=1,2$. Como (1) é válido para qualquer processo de variação limitada,

$$
\begin{gathered}
\mathbb{E} B_{\infty}^{2}=2 \mathbb{E} \sum_{k=0}^{\infty} \mathbb{E}\left[B_{\infty}-B_{k} \mid \mathcal{F}_{k}\right] b_{k}-\mathbb{E} \sum_{k=0}^{\infty} b_{k}^{2} \\
\leq \mathbb{E} \sum_{k=0}^{\infty} \mathbb{E}\left[W \mid \mathcal{F}_{k}\right]\left(a_{k}^{(1)}+a_{k}^{(2)}\right) \leq \mathbb{E}\left[W\left(A_{\infty}^{(1)}+A_{\infty}^{(2)}\right)\right] .
\end{gathered}
$$

Aplicando a desigualdade de Cauchy-Schwarz e os limitantes para $\mathbb{E}\left(A_{\infty}^{(i)}\right)^{2}$ obtemos

$$
\mathbb{E} B_{\infty}^{2} \leq c N\left(\mathbb{E} W^{2}\right)^{1 / 2}
$$

Considerando o limite em $L^{2}$ sobre o supremo dos $B_{k} s$, sejam $M_{k}=\mathbb{E}\left[B_{\infty} \mid \mathcal{F}_{k}\right], N_{k}=\mathbb{E}\left[W \mid \mathcal{F}_{k}\right]$, e $X_{k}=M_{k}-B_{k}$. Temos que

$$
\left|X_{k}\right|=\left|\mathbb{E}\left[B_{\infty}-B_{k} \mid \mathcal{F}_{k}\right]\right| \leq N_{k}
$$

pela desigualdade de Doob,

$$
\mathbb{E}\left[\sup _{k} X_{k}^{2}\right] \leq \mathbb{E}\left[\sup _{k} N_{k}^{2}\right] \leq c \mathbb{E} N_{\infty}^{2}=c \mathbb{E} W^{2}
$$

Novamente, pela desigualdade de Doob,

$$
\mathbb{E}\left[\sup _{k} M_{k}^{2}\right] \leq c \mathbb{E} M_{\infty}^{2}=c \mathbb{E} B_{\infty}^{2}
$$


Como $\sup _{k}\left|B_{k}\right| \leq \sup _{k}\left|X_{k}\right|+\sup _{k}\left|M_{k}\right|, \log \mathrm{O}$

$$
\mathbb{E}\left[\sup _{k} B_{k}^{2}\right] \leq c \mathbb{E} W^{2}+c N\left(\mathbb{E} W^{2}\right)^{1 / 2}
$$

Aqui está a decomposição de Doob-Meyer no caso de tempo discreto:

(4.21) Proposição: $S e Z_{k}$ é um supermartingal de tempo discreto, existe um martingal $M_{k}$ e um processo crescente $A_{k}$ tal que $A_{k+1}$ é $\mathcal{F}_{k}$-mensurável e $Z_{k}=M_{k}-A_{k}$.

Demonstração: Seja $a_{k}=\mathbb{E}\left[Z_{k}-Z_{k+1} \mid \mathcal{F}\right]$. Como $Z$ é um supermartingal, os $a_{k}$ são não negativos e claramente $\mathcal{F}_{k}$-mensuráveis. Seja $A_{k}=\sum_{j=1}^{k-1} a_{j}$, é fácil ver que $Z_{k}+A_{k}$ é um martingal.

Vamos provar agora a existência no teorema (4.17):

Demonstração: Como $Z_{t}$ é contínuo, é suficiente mostrar que $Z_{t \wedge T_{N}}$ tem a decomposição desejada para cada $N$, onde $T_{N}=\inf \left\{t:\left|Z_{t}\right|>N\right\}$, em seguida fazer $N \longrightarrow \infty$, e usar a unicidade. Assim, podemos assumir que $Z$ é limitado. Além disso, se nós temos a decomposição para qualquer tempo fixado $M$, podemos fazer $M \longrightarrow \infty$, e obter o resultado de existência. Logo podemos assumir que $Z_{t}$ é constante para $t \geq M$, e assim que q.s. as trajetórias de $Z_{t}$ são uniformemente contínuas.

Fixemos $M$ e $n$, seja $\mathcal{F}_{k}^{n}=\mathcal{F}_{k / 2^{n}}$. Após construir $A_{k}^{n}$ através da proposição (4.21), seja $B_{t}^{n}=A_{k}^{n}$ e $\mathcal{G}_{t}^{n}=\mathcal{F}_{k}^{n}$ se $(k-1) / 2^{n}<t \leq k / 2^{n}$.

Seja $W(\delta)=\sup _{s \leq M, s \leq t \leq s+\delta}\left|Z_{t}-Z_{s}\right|$. Como $Z$ é limitado, temos que $W(\delta)$ também é. Uma vez que as trajetórias de $Z$ são uniformemente contínuas, $W(\delta) \longrightarrow 0$ q.s. quando $\delta \longrightarrow 0$. Assim $W(\delta) \longrightarrow 0$ em $L^{2}$. 
Devemos primeiro provar que $B_{k}^{n}$ converge em $L^{2}$ quando $n \longrightarrow \infty$, uniformemente sobre $t$. Para provar isto temos que mostrar que

$$
\mathbb{E}\left[\sup _{t}\left|B_{t}^{m}-B_{t}^{n}\right|^{2}\right] \longrightarrow 0
$$

quando $n, m \longrightarrow \infty$. Logo $B_{k}^{n}$ converge pois é sequência de Cauchy. Vamos estimar a norma em $L^{2}$ do supremo da diferença usando o lema (4.20). Supondo $m \geq n, B_{t}^{n}$ e $B_{t}^{m}$ são constantes sobre os intervalos $\left(k / 2^{m},(k+\right.$ 1) $/ 2^{m}$ ], o supremo da diferença será alcançado em algum $k / 2^{m}$. Fixe $t=$ $k / 2^{m}$ para algum $k$, e assim a diferença das esperanças condicionais com respeito a $\mathcal{F}_{k}^{m}$ estará limitada. Seja $u$ o menor múltiplo de $2^{-n}$ maior do que ou igual a $t$. Pela proposição (4.21):

$$
\mathbb{E}\left[B_{\infty}^{m}-B_{t}^{m} \mid \mathcal{G}_{t}^{m}\right]=\mathbb{E}\left[A_{\infty}^{m}-A_{k}^{m} \mid \mathcal{F}_{k / 2^{m}}\right]=\mathbb{E}\left[Z_{t}-Z_{\infty} \mid \mathcal{F}_{t}\right]
$$

Por outro lado,

$$
\begin{gathered}
\mathbb{E}\left[B_{\infty}^{n}-B_{t}^{n} \mid \mathcal{G}_{t}^{m}\right]=\mathbb{E}\left[A_{\infty}^{n}-A_{u 2^{n}}^{n} \mid \mathcal{F}_{t}\right] \\
=\mathbb{E}\left[\mathbb{E}\left[A_{\infty}^{n}-A_{u 2^{n}}^{n} \mid \mathcal{F}_{u}\right] \mid \mathcal{F}_{t}\right]=\mathbb{E}\left[\mathbb{E}\left[Z_{u}-Z_{\infty} \mid \mathcal{F}_{u}\right] \mid \mathcal{F}_{t}\right]=\mathbb{E}\left[Z_{u}-Z_{\infty} \mid \mathcal{F}_{t}\right],
\end{gathered}
$$

onde usamos a definição de $A_{k}^{n}$. Assim a diferença das esperanças condicionais é limitada:

$$
\left|\mathbb{E}\left[B_{\infty}^{m}-B_{t}^{m} \mid \mathcal{F}_{t}\right]-\mathbb{E}\left[B_{\infty}^{n}-B_{t}^{n} \mid \mathcal{F}_{t}\right]\right| \leq \mathbb{E}\left[\left|Z_{t}-Z_{u}\right| \mid \mathcal{F}_{t}\right] \leq \mathbb{E}\left[W\left(2^{-n}\right) \mid \mathcal{F}_{t}\right] .
$$

Pelo lema (4.20) temos que $\mathbb{E}\left[\sup _{t}\left|B_{\infty}^{n}-B_{t}^{n}\right|^{2}\right]$ tende a zero quando $n \longrightarrow \infty$.

Vamos agora mostrar que o limite é contínuo. Os saltos de $B_{t}^{n}$ são

$$
\Delta B_{t}^{n}=\mathbb{E}\left[Z_{(k-1) / 2^{n}}-Z_{k / 2^{n}} \mid \mathcal{F}_{(k-1) / 2^{n}}\right], t=k / 2^{n},
$$


o qual é limitado por

$$
\mathbb{E}\left[W\left(2^{-n}\right) \mid \mathcal{F}_{(k-1) / 2^{n}}\right]
$$

Assim

$$
\begin{gathered}
\mathbb{E}\left[\sup _{t}\left(\Delta B_{t}^{n}\right)^{2}\right] \leq \mathbb{E}\left[\sup _{k}\left(\mathbb{E}\left[W\left(2^{-n}\right) \mid \mathcal{F}_{(k-1) / 2^{n}}\right]\right)^{2}\right] \\
\leq c \mathbb{E}\left[W\left(2^{-n}\right)\right]^{2} \longrightarrow 0
\end{gathered}
$$

pela desigualdade de Doob, como $\mathbb{E}\left[W\left(2^{-n}\right) \mid \mathcal{F}_{(k-1) / 2^{n}}\right]$ é um martingal.

Olhando para uma subsequência conveniente $n_{j}, \sup _{t}\left|\Delta A_{j}^{n_{j}}\right| \longrightarrow 0$, q.s., então o limite é contínuo.

Finalmente, só falta mostrar que se $A_{t}$ é o limite de $B_{t}^{n}$, então $Z_{t}+A_{t}$ é um martingal. Como $Z_{t}$ e $A_{t}$ são ambos contínuos e quadrado integráveis, é suficiente mostrar que para todos $s, t \in D_{n}, s<t$, e $B \in \mathcal{F}_{s}, \mathbb{E}\left[Z_{t}+A_{t} ; B\right]=$ $\mathbb{E}\left[Z_{s}+A_{s} ; B\right]$, mas isto é verdade para cada $Z_{t}+B_{t}^{n}$, e o resultado segue passando ao limite e utilizando a desigualdade de Cauchy-Schwarz.

\subsubsection{Variação Quadrática}

Se $M_{t}$ é um martingal contínuo de quadrado integrável (ie, $\mathbb{E} M_{\infty}^{2}<\infty$ ), então $M_{t}^{2}$ é um submartingal e $-M_{t}^{2}$ é um supermartingal. Pelo teorema (4.17) existe um processo contínuo crescente, denotado por $\langle M\rangle_{t}$, e chamado de variação quadrática de $M$, tal que $M_{t}^{2}-\langle M\rangle_{t}$, é um martingal. Dados dois martingais $M, N$, definimos $\langle M, N\rangle_{t}$ por polarização:

$$
\langle M, N\rangle_{t}=\frac{1}{2}\left(\langle M+N\rangle_{t}-\langle M\rangle_{t}-\langle N\rangle_{t}\right)
$$


(4.22) Teorema: Para cada $\epsilon$ suponha que $S_{i}(\epsilon)$ é uma sequência de tempos de parada que tende ao infinito quando $i \longrightarrow \infty$ tal que $\sup _{i} \mid M_{S_{i+1}(\epsilon)}-$ $M_{S_{i}(\epsilon)} \mid \longrightarrow 0$ quando $\epsilon \longrightarrow 0$. Então

$$
\sum_{i=0}^{\infty}\left(M_{S_{i+1}(\epsilon)}-M_{S_{i}(\epsilon)}\right)^{2} \longrightarrow\langle M\rangle_{\infty}
$$

em probabilidade.

Demonstração: Tomando o primeiro tempo em que $M_{t}$ ou $\langle M\rangle_{t}$ excede $N$, podemos assumir que $M$ e $\langle M\rangle$ são ambos limitados. Para não carregar a notação vamos omitir $(\epsilon)$ de agora em diante:

Vamos aplicar o lema (4.20), sejam $a_{i}^{(1)}=\left(M_{S_{i+1}}-M_{S_{i}}\right)^{2}, a_{i}^{(2)}=\left(\langle M\rangle_{S_{i+1}}-\right.$ $\left.\langle M\rangle_{S_{i}}\right)^{2}, b_{i}=a_{i}^{(1)}-a_{i}^{(2)}$. Sejam $A^{(1)}, A^{(2)}$ as respectivas somas dos $a_{i} \mathrm{~s}$, e seja $\mathcal{F}_{k}=\sigma\left(M_{S_{i+1}}, i \leq k\right)$. Como $M$ e $\langle M\rangle$ são limitados, é claro que as hipóteses do lema (4.20) estão satisfeitas para $A^{(1)}$ e $A^{(2)}$. Seja $B_{k}=A_{k}^{(1)}-A_{k}^{(2)}$. Seja

$$
W(\epsilon)=\sup _{i}\left(\left|\left(M_{S_{i+1}}-M_{S_{i}}\right)\right|^{2}+\langle M\rangle_{S_{i+1}}-\langle M\rangle_{S_{i}}\right) .
$$

Temos $B_{\infty}-B_{k}=\sum_{i=k}^{\infty} b_{i}$. Note que $\mathbb{E}\left[b_{i} \mid \mathcal{F}_{k}\right]=0$ se $i>k$. Assim,

$$
\left|\mathbb{E}\left[B_{\infty}-B_{k} \mid \mathcal{F}_{k}\right]\right| \leq a_{k}^{(1)}-a_{k}^{(2)}
$$

Observe que $\sup _{k} a_{k}^{(1)} \leq \sup _{k} \mathbb{E}\left[W(\epsilon) \mid \mathcal{F}_{k}\right]$ e analogamente para $\sup _{k} a_{k}^{(2)}$. Assim aplicando o lema (4.20), nós obtemos o limite

$$
\left.\mathbb{E}\left[\sup _{k} B_{k}^{2}\right] \leq c\left(\mathbb{E} W(\epsilon)^{2}\right)^{1 / 2}+\mathbb{E} W(\epsilon)^{2}\right)
$$

o qual vai a zero quando $\epsilon \longrightarrow 0$.

Seja $X_{t}$ um processo $\mathcal{F}_{t}$-mensurável e não negativo. Para cada $\omega \in \Omega$ e $t \in \mathbb{R}$, a integral de Lebesgue-Stieltjes 


$$
Y(t, \omega)=\int_{0}^{t} X(s, \omega) d\langle M\rangle_{s}
$$

existe e o processo $Y$ será adaptado e mensurável em relação a $\mathcal{F}_{t}$. Uma construção detalhada desta integral pode vista em [niski][13].

\subsection{Movimento Browniano}

\subsubsection{Preliminares}

Agora vamos definir o processo estocástico que é a principal personagem nesta dissertação, o Movimento Browniano.

(4.23) Definição: Um processo estocástico $X_{t}$ é um movimento browniano padrão unidimensional se:

(i) $X_{0}=0$ com probabilidade 1 ;

(ii)Para todos $s \leq t, X_{t}-X_{s}$ é uma variável aleatória normal com média 0 e variância $t-s$;

(iii)Para todo $s<t, X_{t}-X_{s}$ é independente de $\sigma\left(X_{r} ; r \leq s\right)$;

(iv)Com probabilidade 1 a função $t \longrightarrow X_{t}(\omega)$ é contínua.

Por definição, $\sigma\left(X_{r} ; r \leq s\right)$ é a menor $\sigma$-álgebra na qual todo $X_{r}, r \leq s$, é mensurável.

Existem diversas demonstrações de existência e unicidade do movimento browniano que podem ser encontradas por exemplo em livros como [durrett1][6] e [bass][2].

Não é claro por exemplo, que as propriedades (ii) e (iii) sejam consistentes com (iv), se tivéssemos suposto que ao invés da distribuição normal em (ii) a distribuição fosse de Cauchy teríamos como consequência que as trajetórias seriam descontínuas com probabilidade um, o que nos mostra que a questão de existência é não trivial (veja [durrett1][6], página 2). 
Uma versão da propriedade (iii) que vamos utilizar bastante é

(iii')Se $0 \leq t_{0}<t_{1}<\ldots<t_{k}$ então $X_{t_{i}}(\omega)-X_{t_{i-1}}(\omega)$ são independentes $(1 \leq i \leq k)$.

Vamos mostrar que (iii) implica em (iii'):

Sejam $A_{i} \in \sigma\left(X_{t_{i}}-X_{t_{i-1}}\right),(1 \leq i \leq k)$. Sabemos que $\sigma\left(X_{t_{k}}-X_{t_{k-1}}\right)$ é independente de $\sigma\left(X_{r} ; r \leq t_{k-1}\right)$. Temos

$$
A_{i} \in \sigma\left(X_{t_{i}}-X_{t_{i-1}}\right) \subset \sigma\left(X_{r} ; r \leq t_{k-1}\right) \Rightarrow \cap_{i=1}^{k-1} A_{i} \in \sigma\left(X_{r}, r \leq t_{k-1}\right) .
$$

Assim $\mathbb{P}\left(\cap_{i=1}^{k} A_{i}\right)=\mathbb{P}\left(A_{k} \cap\left(\cap_{i=1}^{k-1} A_{i}\right)\right)=\mathbb{P}\left(A_{k}\right) \cdot \mathbb{P}\left(\cap_{i=1}^{k-1} A_{i}\right)$ e daí segue por indução que $\mathbb{P}\left(\cap_{i=1}^{k} A_{i}\right)=\prod_{i=1}^{k} \mathbb{P}\left(A_{i}\right)$.

Este movimento browniano é unidimensional, para definir o movimento browniano $d$-dimensional, sejam $X_{t}^{1}, \ldots X_{t}^{d}$ movimentos brownianos unidimensionais independentes. Temos que $X_{t}=\left(X_{t}^{1}, \ldots X_{t}^{d}\right)$ é um movimento browniano $d$-dimensional.

Como $X_{0}=0$ temos que este movimento browniano começa em 0 , para considerar um movimento iniciando em $x \in \mathbb{R}^{d}$ podemos simplesmente considerar $x+X_{t}$. No entanto, ao invés de considerar $\mathbb{P}$ e $X_{t}^{x}=x+X_{t}$, ou seja uma medida de probabilidade e muitos processos, é mais interessante considerar um processo e muitas medidas de probabilidade.

Seja $Z_{t}$ um movimento browniano $d$-dimensional definido como anteriormente. Seja $\Omega$ o conjunto de funções contínuas $\omega$ de $[0, \infty)$ em $\mathbb{R}^{d}$ (não estamos exigindo que $\omega(0)=0$ aqui). Cada elemento $\omega$ em $\Omega$ é assim uma função contínua. Tome $X_{t}(\omega)=\omega(t)$.

No processo $X_{t}$ acima definido seja $\mathcal{F}=\sigma\left(X_{r} ; r<\infty\right)$. Agora defina $\mathbb{P}^{x}$ sendo a medida de probabilidade dada por

$$
\mathbb{P}^{x}(A)=\mathbb{P}(A-x)
$$

onde $x \in \mathbb{R}^{d}, A \in \mathcal{F}, A-x=\{\omega \in \Omega ; \omega()+.x \in A\}, \omega()+x:.[0, \infty) \longrightarrow \mathbb{R}^{d}$ e $(\omega()+x).(t)=\omega(t)+x$ (trajetória transladada). 
O par $\left(\mathbb{P}^{x}, X_{t}\right), x \in \mathbb{R}, t \geq 0$ é chamado um movimento browniano.

Vamos denotar a esperança em relação a $\mathbb{P}^{x}$ como $\mathbb{E}^{x}$.

As trajetórias do movimento browniano são extremamente irregulares, com probabilidade um, são não diferenciáveis em quase todo ponto. Este resultado é devido a [paley][14], uma prova mais simples foi dada por [dvoretsky][8].

Vamos algumas propriedades do movimento browniano.

(4.24) Proposição: Se um processo gaussiano $\left\{X_{t}: 0 \leq t<\infty\right\}$ tem $\mathbb{E}\left[X_{t}\right]=0$ para todo $t$ e se $\operatorname{Cov}\left(X_{s}, X_{t}\right)=s \wedge t, \forall s, t, 0 \leq s, t$, então o processo $X_{t}$ tem incrementos independentes. Mais ainda se o processo tem caminhos contínuos e $X_{0}=0$, então ele é o movimento browniano padrão.

Demonstração: Considere o vetor aleatório de incrementos independentes do processo $X_{t},\left(X_{t_{2}}-X_{t_{1}}, X_{t_{3}}-X_{t_{2}}, \ldots, X_{t_{n}}-X_{t_{n-1}}\right)$, este tem matriz de covariâncias diagonal.

Note que para $i<j$ :

$$
\begin{gathered}
\mathbb{E}\left[\left(X_{t_{i}}-X_{t_{i-1}}\right)\left(X_{t_{j}}-X_{t_{j-1}}\right)\right]=\mathbb{E}\left[X_{t_{i}} X_{t_{j}}\right]-\mathbb{E}\left[X_{t_{i}} X_{t_{j-1}}\right]-\mathbb{E}\left[X_{t_{i-1}} X_{t_{j}}\right]+ \\
\mathbb{E}\left[X_{t_{i-1}} X_{t_{j-1}}\right]=t_{i}-t_{i}-t_{i-1}+t_{i-1}=0
\end{gathered}
$$

e a segunda parte segue da definição do movimento browniano.

Vamos ver mais duas propriedades interessantes do movimento browniano, para começar, a de escala:

(4.25) Proposição: $S e\left(\mathbb{P}^{x}, X_{t}\right)$ é um movimento browniano iniciando em $x$ e $a>0$, então $\left(\mathbb{P}^{x / a}, a^{-1} X_{a^{2} t}\right)$ é um movimento browniano iniciando em $x / a$. 
Demonstração: Vamos fazer o caso unidimensional, o caso multidimensional é análogo. Como $\mathbb{P}^{x}$ é definida por translação, é suficiente considerar o caso $x=0$. Temos que $a^{-1} X_{a^{2} t}$ é contínuo em $t$, e

$$
\operatorname{Cov}\left(a^{-1} X_{a^{2} t}, a^{-1} X_{a^{2} s}\right)=a^{-2}\left(a^{2} t \wedge a^{2} s\right)=s \wedge t
$$

Se $t_{1} \leq t_{1} \leq \ldots \leq t_{n}$, a lei de distribuição de

$$
\left(a^{-1} X_{a^{2} t_{1}}, \ldots, a^{-1} X_{a^{2} t_{n}}\right)
$$

é uma gaussiana multivariada, pela proposição anterior $a^{-1} X_{a^{2} t}$ deve ser um movimento browniano.

Outra propriedade interessante é que o movimento browniano é invariante sob rotações.

(4.26) Proposição: Se A é uma matriz ortogonal, então $\left(\mathbb{P}^{A x}, A X_{t}\right)$ é um movimento browniano iniciando em Ax.

Demonstração: Considere o caso em que $x=0$ : se $A=\left(a_{i j}\right)$ e $X_{t}=$ $\left(X_{t}^{1}, \ldots, X_{t}^{d}\right)$ temos $A X_{t}^{T}=\left(\sum_{k=1}^{d} a_{1 k} X_{t}^{k}, \ldots, \sum_{k=1}^{d} a_{d k} X_{t}^{k}\right)^{T}$.

Temos que $A X_{t}^{T}$ tem caminhos contínuos, começa em $x=0$, tem média zero, e como é combinação linear de processos gaussianos é gaussiano.

$\operatorname{Cov}\left(\sum_{k=1}^{d} a_{m k} X_{t}^{k}, \sum_{k=1}^{d} a_{n k} X_{t}^{k}\right)=\mathbb{E}\left[\sum_{k=1}^{d} a_{m k} X_{t}^{k} \cdot \sum_{k=1}^{d} a_{n k} X_{t}^{k}\right]=$ $\sum_{i \neq j} b_{i j} \mathbb{E}\left[X_{t}^{i} X_{t}^{j}\right]+\sum_{i=1}^{d} b_{i i} \mathbb{E}\left[X_{t}^{i} X_{t}^{i}\right]=0+0=0$.

Assim temos que $A X_{t}^{T}$ é um processo com incrementos independentes.

$$
\begin{aligned}
& \operatorname{Cov}\left(\sum_{k=1}^{d} a_{m k} X_{s}^{k}, \sum_{k=1}^{d} a_{m k} X_{t}^{k}\right)=\mathbb{E}\left[\sum_{k=1}^{d} a_{m k} X_{s}^{k} \cdot \sum_{k=1}^{d} a_{m k} X_{t}^{k}\right]= \\
& \sum_{i \neq j} b_{i j} \mathbb{E}\left[X_{s}^{i} X_{t}^{j}\right]+\sum_{i=1}^{d} b_{i i} \mathbb{E}\left[X_{s}^{i} X_{t}^{i}\right]=1 .(s \wedge t)=s \wedge t .
\end{aligned}
$$




\subsubsection{O Movimento Browniano é um Processo Markoviano}

Seja $\mathcal{F}_{t}^{00}=\sigma\left(X_{s} ; s \leq t\right), t \in[0, \infty]$. Seja $\mathcal{F}_{t}^{0}$ o completamento de $\mathcal{F}_{t}^{00}$, porém aqui é necessário ser cuidadoso com o significado de completamento, pois estamos trabalhando com mais de uma medida de probabilidade. Seja $\mathcal{F}_{t}^{0}$ a $\sigma$-álgebra gerada por $\mathcal{F}_{t}^{00}$ e $\mathcal{N}$, onde $\mathcal{N}$ é a coleção de conjuntos que têm medida nula segundo $\mathbb{P}^{x}$ para todo $x \in \mathbb{R}^{d}$. Finalmente, seja

$$
\mathcal{F}_{t}=\mathcal{F}_{t+}^{0}=\cap_{\epsilon>0} \mathcal{F}_{t+\epsilon}^{0} .
$$

Note que $\left\{\mathcal{F}_{t}\right\}_{t}$ é uma filtração contínua à direita por construção.

Nós vamos utilizar somente $\mathcal{F}_{t}$ mas precisamos das outras em estágios intermediários, enquanto $\mathcal{F}_{t}^{00}$ é pequena demais para incluir muitos conjuntos interessantes, outras filtrações podem ser grandes demais para que a propriedade de Markov seja válida.

Se $\Omega$ é o conjunto de funções contínuas de $[0, \infty)$ em $\mathbb{R}^{d}$, nós definimos os operadores deslocamento ("shift operators") $\theta_{t}: \Omega \longrightarrow \Omega$ por

$$
\theta_{t}(\omega)(s)=\omega(t+s)
$$

Então

$$
X_{s} \circ \theta_{t}(\omega)=X_{s}\left(\theta_{t} \omega\right)=\theta_{t} \omega(s)=\omega(s+t)=X_{t+s}(\omega)
$$

se o processo é dado por funções coordenadas como em (4.23). Podemos tomar $t$ tal que

$$
X_{s+t}=X_{s} \circ \theta_{t}
$$

Definimos $\mathbb{E}^{X_{s}} Y$ como sendo a variável aleatória $\varphi\left(X_{s}\right)$, onde $\varphi(y)=\mathbb{E}^{y} Y$.

Vejamos a propriedade de Markov para $\mathcal{F}_{t}^{00}$. 
(4.27) Proposição: Se $Y$ é limitada e $\mathcal{F}_{t}^{00}$-mensurável e $\left(\mathbb{P}^{x}, X_{t}\right)$ é um movimento browniano, então

$$
\mathbb{E}^{x}\left[Y \circ \theta_{s} \mid \mathcal{F}_{s}^{00}\right]=\mathbb{E}^{X_{s}} Y, \text { q.s. }\left(\mathbb{P}^{x}\right) .
$$

Vejamos esta proposição aplicada no caso particular em que $Y=f\left(X_{t}\right)$, onde $f$ é limitada e Borel mensurável. Temos

$$
\begin{gathered}
\mathbb{E}^{x}\left[f\left(X_{t+s}\right) \mid \mathcal{F}_{s}^{00}\right]=\mathbb{E}^{x}\left[f\left(X_{t} \circ \theta_{s}\right) \mid \mathcal{F}_{s}^{00}\right]=\mathbb{E}^{x}\left[f \circ\left(X_{t} \circ \theta_{s}\right) \mid \mathcal{F}_{s}^{00}\right]= \\
\mathbb{E}^{x}\left[\left(f \circ X_{t}\right) \circ \theta_{s} \mid \mathcal{F}_{s}^{00}\right]=\mathbb{E}^{x}\left[f\left(X_{t}\right) \circ \theta_{s} \mid \mathcal{F}_{s}^{00}\right]=\mathbb{E}^{X_{s}}\left[f\left(X_{t}\right)\right]
\end{gathered}
$$

Vamos provar a propriedade de Markov para este caso particular, o caso geral pode ser encontrado em [durrett2][7]. Assim, temos:

(4.28) Proposição: Se f é limitada e Borel mensurável,

$$
\mathbb{E}^{x}\left[f\left(X_{t}\right) \circ \theta_{s} \mid \mathcal{F}_{s}^{00}\right]=\mathbb{E}^{X_{s}}\left[f\left(X_{t}\right)\right] \text { q.s. }\left(\mathbb{P}^{x}\right) .
$$

Demonstração: Seja $g(y, z)=f(y+z)$, vamos precisar do seguinte lema:

(4.29) Lema: Se g (como definida anteriormente) é limitada então

$$
\mathbb{E}^{x}\left[g\left(X_{s}, X_{t+s}-X_{s}\right) \mid \mathcal{F}_{s}^{00}\right]=\varphi_{g}\left(X_{s}\right)
$$

onde $\varphi_{g}(y)=\int_{\mathbb{R}} g(y, z)(2 \pi t)^{-d / 2} e^{-|z|^{2} / 2 t} d z$. 
Demonstração: Para provar este lema, considere inicialmente o caso em que $g(y, z)=g_{1}(y) g_{2}(z)$, temos

$$
\varphi_{g}(y)=g_{1}(y) \int g_{2}(z)(2 \pi t)^{-d / 2} e^{-|z|^{2} / 2 t} d z
$$

Note que

$$
\begin{gathered}
\mathbb{E}^{x}\left[g\left(X_{s}, X_{t+s}-X_{s}\right) \mid \mathcal{F}_{s}^{00}\right]=\mathbb{E}^{x}\left[g_{1}\left(X_{s}\right) g_{2}\left(X_{t+s}-X_{s}\right) \mid \mathcal{F}_{s}^{00}\right]= \\
g_{1}\left(X_{s}\right) \mathbb{E}^{x}\left[g_{2}\left(X_{t+s}-X_{s}\right) \mid \mathcal{F}_{s}^{00}\right]=g_{1}\left(X_{s}\right) \mathbb{E}^{x}\left[g_{2}\left(X_{t+s}-X_{s}\right)\right]= \\
g_{1}\left(X_{s}\right) \int_{\mathbb{R}} g_{2}(z)(2 \pi t)^{-d / 2} e^{-|z|^{2} / 2 t} d z=\varphi_{g}\left(X_{s}\right)
\end{gathered}
$$

Vamos agora provar o lema (4.29) estendendo o caso inicial para todas as funções mensuráveis limitadas. Vamos precisar do seguinte resultado de Teoria da Medida:

(4.30) Teorema da Classe Monótona: Seja $\mathcal{A}$ uma coleção de subconjuntos de $\Omega$ que contém $\Omega$ e é fechado sob intersecção finita. Seja $\mathcal{H}$ o espaço vetorial de funções $f: \Omega \longrightarrow \mathbb{R}^{d}$ tais que

(i) $A \in \mathcal{A} \Rightarrow 1_{A} \in \mathcal{H}$;

(ii) $g_{n} \in \mathcal{H}$ são não negativas e crescentes a g limitada implica que $g \in \mathcal{H}$.

Logo $\mathcal{H}$ contém todas as funções que são mensuráveis em relação à $\sigma$-álgebra gerada por $\mathcal{A}$.

Seja $\mathcal{A}$ o conjunto de todos os retângulos $A \times B$ onde $A, B$ são retângulos de $\mathbb{R}^{d}$ e $\mathcal{H}$ o conjunto das funções limitadas $g$ onde vale (4.29). Claramente $\mathcal{H}$ forma um espaço vetorial e dado $(A \times B) \in \mathcal{A}, 1_{A \times B}=1_{A} \cdot 1_{B}=g_{1} . g_{2}=g \in$ 
$\mathcal{H}, \log$ vale (i).

Se $g_{n} \in \mathcal{H}$, pelo teorema da convergência monótona temos:

$$
\begin{gathered}
\mathbb{E}^{x}\left[g\left(X_{s}, X_{t+s}-X_{s}\right) \mid \mathcal{F}_{s}^{00}\right]=\lim _{n \longrightarrow \infty} \mathbb{E}^{x}\left[g_{n}\left(X_{s}, X_{t+s}-X_{s}\right) \mid \mathcal{F}_{s}^{00}\right]= \\
\lim _{n \longrightarrow \infty} \varphi_{g_{n}}\left(X_{s}\right)=\lim _{n \longrightarrow \infty} \int g_{n}(y, z)(2 \pi t)^{-d / 2} e^{-|z|^{2} / 2 t} d y= \\
\int g(y, z)(2 \pi t)^{-d / 2} e^{-|z|^{2} / 2 t} d y=\varphi_{g}\left(X_{s}\right) \Rightarrow g \in \mathcal{H}
\end{gathered}
$$

Logo vale o lema (4.29).

Vamos finalmente provar a proposição (4.28).

Basta tomar $g\left(X_{s}, X_{t+s}-X_{s}\right)=f\left(X_{t+s}\right)$ e observar que

$$
\varphi_{g}\left(X_{s}\right)=\int g\left(X_{s}, X_{t+s}-X_{s}\right)(2 \pi t)^{-d / 2} e^{-|z|^{2} / 2 t} d y=\mathbb{E}^{x}\left[f\left(X_{t}\right)\right]
$$

Acrescentar conjuntos de medida nula não altera em nada a demonstração (conforme [bass][2]), logo temos: 
(4.31) Proposição: Se Y é limitada e $\mathcal{F}_{\infty}^{0}$-mensurável, então

$$
\mathbb{E}^{x}\left[Y \circ \theta_{t} \mid \mathcal{F}_{t}^{0}\right]=\mathbb{E}^{X_{t}} Y \text { q.s. }\left(\mathbb{P}^{x}\right) .
$$

Seja

$$
p(t, x, y)=(2 \pi t)^{-d / 2} e^{-|x-y|^{2} / 2 t},
$$

$x, y \in \mathbb{R}^{d}, t>0$. O correspondente operador $P_{t}$ sobre funções é dado por

$$
P_{t} f(x)=\int f(y) p(t, x, y) d y .
$$

Note que se $f$ é limitada e $t>0$, então $P_{t} f(x)$ é contínua em $x$ pela continuidade de $p(t, x, y)$ e pelo teorema de convergência dominada.

(4.32) Teorema: Se Y é limitada e $\mathcal{F}_{\infty}$-mensurável e $\left(\mathbb{P}^{x}, X_{t}\right)$ é um movimento browniano,

$$
\mathbb{E}^{x}\left[Y \circ \theta_{t} \mid \mathcal{F}_{t}\right]=\mathbb{E}^{X_{t}} Y .
$$

Demonstração: Vamos ver o caso em que $Y=f\left(X_{t}\right)$, o caso geral pode ser visto em [durrett2][7]. Por meio de um processo limite, podemos assumir que $f$ é contínua.

Se $A \in \mathcal{F}_{s}=\mathcal{F}_{s+}^{0}$ para todo $\epsilon>0$. Assim pela propriedade de Markov com respeito a $\mathcal{F}_{s+\epsilon}^{0}$,

$$
\mathbb{E}^{x}\left[f\left(X_{t+s+\epsilon}\right) ; A\right]=\mathbb{E}^{x}\left[P_{t} f\left(X_{s+\epsilon}\right) ; A\right] .
$$

Se fizermos $\epsilon \longrightarrow 0$, o lado esquerdo converge para $\mathbb{E}^{x}\left[f\left(X_{t+s}\right) ; A\right]$ por convergência dominada e continuidade de $f$ e $X$. Como observado $P_{t} f$ é contínuo.

O lado direito converge para $\mathbb{E}^{x}\left[P_{t} f\left(X_{s}\right) ; A\right]$ por convergência dominada e a 
continuidade de $P_{t} f$ e $X$.

\subsubsection{Leis $0-1$}

(4.33) Proposição: Temos que $\mathcal{F}_{s}=\mathcal{F}_{s}^{0}$.

Demonstração: Seja $Y=f_{j}\left(X_{t_{j}}\right)$. Se $t_{j} \leq s<t_{j+1}$, sejam

$Y_{1}=\prod_{\left\{j: t_{j} \leq s\right\}} f_{j}\left(X_{t_{j}}\right)$ e $Y_{2}=\prod_{\left\{j: t_{j}>s\right\}} f_{j}\left(X_{t_{j}}\right)$. Então

$$
\mathbb{E}^{x}\left[Y \mid \mathcal{F}_{s+}^{0}\right]=Y_{1} \mathbb{E}^{X_{s}} Y_{2},
$$

o qual é $\mathcal{F}_{s}^{0}$-mensurável.

Por linearidade e limites (usando o teorema de extensão da classe monótona), $\mathbb{E}^{x}\left[Y \mid \mathcal{F}_{s+}^{0}\right]$ é $\mathcal{F}_{s}^{0}$-mensurável quando $Y \in \mathcal{F}_{\infty}^{0}$. Se $A \in \mathcal{F}_{s}=\mathcal{F}_{s+}^{0}$, fazendo $Y=1_{A}$ temos que $A \in \mathcal{F}_{s}^{0}$.

(4.34) Corolário (Lei 0-1 de Blumenthal): $S e A \in \mathcal{F}_{0}$, então $\mathbb{P}^{x}(A)$ é igual a 0 ou 1 .

Demonstração: Se $A \in \mathcal{F}_{0}$, então considerando $\mathbb{P}^{x}$,

$$
1_{A}=\mathbb{E}^{x}\left[1_{A} \mid \mathcal{F}_{0+}\right]=\mathbb{E}^{X_{0}} 1_{A}=\mathbb{E}^{x} 1_{A}=\mathbb{P}^{x}(A) .
$$

$\operatorname{Assim} \mathbb{P}^{x}(A)=1_{A} \in\{0,1\}$.

Como aplicação da lei 0-1, temos: 
(4.35) Proposição: Temos que

$$
\mathbb{P}^{0}\left(T_{(0, \infty)}=0\right)=1 .
$$

Demonstração: Para qualquer $t$,

$$
\mathbb{P}^{0}\left(T_{(0, \infty)} \leq t\right) \geq \mathbb{P}^{0}\left(X_{t}>0\right)
$$

que pela simetria da distribuição $\mathcal{N}(0, t)$ é $1 / 2$. Fazendo $t \downarrow 0, \mathbb{P}^{0}\left(T_{(0, \infty)}=\right.$ $0) \geq 1 / 2$. Pela lei $0-1$ temos $\mathbb{P}^{0}\left(T_{(0, \infty)}=0\right)=1$.

Este resultado nos diz que o movimento browniano entra imediatamente no eixo real positivo, por simetria ele também entra imediatamente no eixo real negativo. O que está ocorrendo é que o movimento browniano oscila entre os eixos positivo e negativo em toda vizinhança da origem no eixo temporal.

\subsubsection{Propriedade Forte de Markov para o Movimento Browniano}

Dado um tempo de parada $T$, seja a seguinte $\sigma$-álgebra de eventos $\mathcal{F}_{T}=$ $\left\{A \in \mathcal{F}_{\infty}: A \cap(T \leq t) \in \mathcal{F}_{t}, \forall t>0\right\}$. Defina $\theta_{T}$ por $\theta_{T}(\omega)(t)=\omega(T(\omega)+t)$. Assim, $X_{t} \circ \theta_{T}(\omega)=X_{T(\omega)+t}(\omega)$ e $X_{T}(\omega)=X_{T(\omega)}(\omega)$.

(4.36) Teorema: Se $Y$ é limitada e $\mathcal{F}_{\infty}$-mensurável e $\left(\mathbb{P}^{x}, X_{t}\right)$ é um movimento browniano, então $\mathbb{E}^{x}\left[Y \circ \theta_{T} \mid \mathcal{F}_{T}\right]=\mathbb{E}^{X_{T}} Y$, q.s. sobre $(T<\infty)$.

Demonstração: É suficiente provar que $\mathbb{E}^{x}\left[f\left(X_{T+t}\right) \mid \mathcal{F}_{T}\right]=\mathbb{E}^{X_{T}} f\left(X_{t}\right)$ para $f$ limitada e contínua, o caso geral segue por meio de extensão (veja [dur-

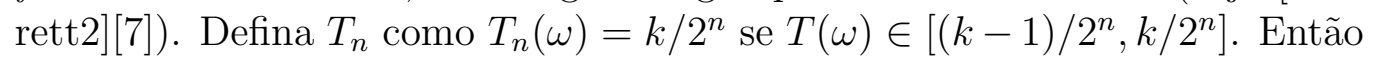
$T_{n}$ são tempos de parada decrescentes para $T$ no conjunto $(T<\infty)$.

Se $A \in \mathcal{F}_{T}$, então $A \in \mathcal{F}_{T_{n}}$. Temos também que $A \cap\left(T_{n}=k / 2^{n}\right) \in \mathcal{F}_{k / 2^{n}}$ e assim 


$$
\begin{gathered}
\mathbb{E}^{x}\left[f\left(X_{T_{n}+t} ; A \cap\left(T_{n}=k / 2^{n}\right)\right]=\mathbb{E}^{x}\left[f\left(X_{t+k / 2^{n}}\right) ; A \cap\left(T=k / 2^{n}\right)\right]\right. \\
=\mathbb{E}^{x}\left[\mathbb{E}^{X_{k / 2^{n}}} f\left(X_{t}\right) ; A \cap\left(T=k / 2^{n}\right)\right]=\mathbb{E}^{x}\left[\mathbb{E}^{X_{T_{n}}} f\left(X_{t}\right) ; A \cap\left(T=k / 2^{n}\right)\right] .
\end{gathered}
$$

Então

$$
\begin{gathered}
\mathbb{E}^{x}\left[f\left(X_{T_{n}+t}\right) ; A \cap(T<\infty)\right]=\sum_{k=1}^{\infty} \mathbb{E}^{x}\left[f\left(X_{T_{n}+t}\right) ; A \cap\left(T_{n}=k / 2^{n}\right)\right] \\
=\sum_{k=1}^{\infty} \mathbb{E}^{x}\left[\mathbb{E}^{X_{T_{n}}} f\left(X_{t}\right) ; A \cap\left(T_{n}=k / 2^{n}\right)\right]=\mathbb{E}^{x}\left[\mathbb{E}^{X_{T_{n}}}\left[f\left(X_{t}\right) ; A \cap(T<\infty)\right] .\right.
\end{gathered}
$$

Fazendo $n \longrightarrow \infty$ temos

$$
\mathbb{E}^{x}\left[f\left(X_{T_{n}+t}\right) ; A \cap(T<\infty)\right] \longrightarrow \mathbb{E}^{x}\left[f\left(X_{T}\right) ; A \cap(T<\infty)\right]
$$

pelo teorema da convergência dominada e a continuidade de $f$ e $X_{t}$. Por outro lado,

$$
\mathbb{E}^{X_{T_{n}}} f\left(X_{t}\right)=P_{t} f\left(X_{T_{n}}\right) \longrightarrow P_{t} f\left(X_{T}\right)=\mathbb{E}^{X_{T}} f\left(X_{t}\right),
$$

desde que $P_{t} f$ é uma função contínua.

\subsubsection{O Movimento Browniano é um Martingal}

Nesta seção vamos tratar com martingais relacionados ao movimento browniano $\left(X_{t}\right)$. Temos que ele é um martingal, pois

$$
\mathbb{E}\left[X_{t} \mid \mathcal{F}_{s}\right]=X_{s}+\mathbb{E}\left[X_{t}-X_{s} \mid \mathcal{F}_{s}\right]=X_{s}+\mathbb{E}\left[X_{t}-X_{s}\right]=X_{s},
$$


usando incrementos independentes.

Como resultado da aplicação das desigualdades de Doob (teoremas) para o movimento browniano obtemos os seguintes resultados:

(4.37) Proposição: Seja $X_{t}$ um movimento browniano. Então se $a, t>0$,

$$
\mathbb{P}\left(\sup _{s \leq t}\left|X_{s}\right| \geq a\right) \leq 2 e^{-a^{2} / 2 t}
$$

Demonstração: Como $e^{b x}$ é convexa, $e^{b X_{t}}$ é um submartingal não negativo. Pelo teorema (4.9)

$$
\mathbb{P}\left(\sup _{s \leq t} X_{s} \geq a\right)=\mathbb{P}\left(\sup _{s \leq t} e^{b X_{s}} \geq e^{a b}\right) \leq e^{-a b} \mathbb{E} e^{b X_{t}} \leq 2 e^{-a b+b^{2} t / 2} .
$$

Tomando $b=a / t$, e repetindo o argumento para $-X_{t}$, e adicionando as duas desigualdades obtemos o resultado.

Vamos calcular a distribuição de saída do movimento browniano do intervalo $[a, b]$.

(4.38) Proposição: Se $a<x<b$, então $\tau_{[a, b]}<\infty$, q.s., $e$

$$
\begin{aligned}
& \mathbb{P}^{x}\left(X\left(\tau_{[a, b]}\right)=a\right)=\frac{b-x}{b-a}, \\
& \mathbb{P}^{x}\left(X\left(\tau_{[a, b]}\right)=b\right)=\frac{x-a}{b-a} .
\end{aligned}
$$


Demonstração: Vamos escrever $\tau$ para $\tau_{[a, b]}$. $X_{t}^{2}-t$ é um martingal, assim pelo teorema (4.7),

$$
\mathbb{E}^{x} X_{\tau \wedge t}^{2}=\mathbb{E}^{x} \tau \wedge t
$$

Para $t \leq \tau,\left|X_{t}\right| \leq|a|+|b|$, assim, pelo lema de Fatou, $\mathbb{E}^{x} \tau \leq(|a|+|b|)^{2}$, ou $\tau<\infty$ q.s. .

Como $X_{t}$ é um martingal, $\mathbb{E}^{x} X_{\tau \wedge t}=x$. Fazendo $t \longrightarrow \infty$ e usando convergência dominada,

$$
x=\mathbb{E}^{x} X_{\tau}=a \mathbb{P}^{x}\left(X_{\tau}=a\right)+b \mathbb{P}^{x}\left(X_{\tau}=b\right) .
$$

Como $\tau<\infty$ q.s.,

$$
1=\mathbb{P}^{x}\left(X_{\tau}=a\right)+\mathbb{P}^{x}\left(X_{\tau}=b\right) .
$$

Resolvendo este sistema de equações lineares nas incógnitas $\mathbb{P}^{x}\left(X_{\tau}=a\right)$ e $\mathbb{P}^{x}\left(X_{\tau}=b\right)$ obtemos o resultado desejado.

Tomando $b \longrightarrow \infty$ e $x>a$, então

$$
\mathbb{P}^{x}\left(T_{\{a\}}<\infty\right)=1
$$

Analisando a demonstração anterior, segue de imediato a seguinte proposição:

(4.39) Proposição: Suponha que $M_{t}$ é um martingal contínuo que sai de $[a, b]$ com probabilidade um, e $M_{0}=x$. Então

$$
\begin{aligned}
& \mathbb{P}\left(M_{\tau}=a\right)=(b-x) /(b-a) e \\
& \mathbb{P}\left(M_{\tau}=b\right)=(x-a) /(b-a) .
\end{aligned}
$$




\section{Cálculo Estocástico}

A drunk man always returns but a drunk bird is lost forever.

Vamos desenvolver agora o Cálculo Estocástico propriamente dito, mais uma vez só desenvolvemos o necessário para nossas aplicações. Estudaremos o caso real aqui, deixando o caso complexo para o próximo capítulo.

\subsection{Integrais Estocásticas}

\subsubsection{Construção}

Sabemos que as trajetórias do movimento browniano são não diferenciáveis em quase todo ponto, isto significa que não devemos esperar que faça sentido $\int f(s) d M_{s}$ por meio de uma integral de Lebesgue-Stieltjes para integrandos gerais $f$. Entretanto é possível definir uma integral estocástica, nosso objetivo aqui será definir $\int H_{s} d M_{s}$, onde $H_{s}=H_{s}(\omega)$ é um processo estocástico conveniente e $M_{s}$ é um martingal contínuo, na maioria dos casos, o movimento browniano.

A classe de integrandos $H_{s}$ é a seguinte: seja $\mathcal{F}_{t}$ uma filtração satisfazendo as condições usuais. Seja $\mathcal{P}$ a $\sigma$-álgebra sobre $\Omega \times[0, \infty)$ gerada por todos os processos contínuos à esquerda $Y_{t}$ que são adaptados a $\mathcal{F}_{t}$. $\mathcal{P}$ é chamada a $\sigma$-álgebra predizível. Nós vamos querer que $H(s, \omega)$ seja $\mathcal{P}$-mensurável. Primeiro vamos querer que

$$
\mathbb{E}\left[\int_{0}^{\infty} H_{s}^{2} d\langle M\rangle_{s}\right]<\infty
$$

e depois enfraquecer esta condição para

$$
\int_{0}^{\infty} H_{s}^{2} d\langle M\rangle_{s}<\infty, q . s . .
$$

Vamos esboçar a definição da integral estocástica no caso do movimento 
browniano inicialmente. Observe que se $H$ é $\mathcal{F}_{a}$-mensurável e $K$ é $\mathcal{F}_{c^{-}}$ mensurável, $X_{t}$ é um movimento browniano, e $a \leq b \leq c \leq d$, então

$$
\mathbb{E}\left[H\left(X_{b}-X_{a}\right) K\left(X_{d}-X_{c}\right)\right]=\mathbb{E}\left[H\left(X_{b}-X_{a}\right) K \mathbb{E}\left[\left(X_{d}-X_{c}\right)\right] \mid \mathcal{F}_{c}\right]=0 .
$$

Além disso, como $X_{t}^{2}-t$ é um martingal, então

$$
\mathbb{E}\left[\left(X_{b}-X_{a}\right)^{2} \mid \mathcal{F}_{a}\right]=\mathbb{E}\left[X_{b}^{2}-X_{a}^{2} \mid \mathcal{F}_{a}\right]=b-a
$$

e assim,

$$
\mathbb{E}\left[H^{2}\left(X_{b}-X_{a}\right)^{2}\right]=\mathbb{E}\left[H^{2} \mathbb{E}\left[\left(X_{b}-X_{a}\right)^{2}\right] \mid \mathcal{F}_{a}\right]=\mathbb{E}\left[H^{2}(b-a)\right] .
$$

Agora, vamos construir $\int_{0}^{1} H_{s} d X_{s}$. Se $H_{s}$ é elementar, ou seja, da forma $H(\omega) 1_{[a, b]}(s)$ onde $H$ é $\mathcal{F}_{a}$-mensurável, então defina $\int_{0}^{1} H_{s} d X_{s}=H\left(X_{b}-X_{a}\right)$. Se $H_{s}$ é simples, isto é, se é uma combinação linear de integrandos elementares, defina $\int_{0}^{1} H_{s} d X_{s}$ por linearidade. Finalmente, se $H_{s}$ satisfaz $\mathbb{E} \int_{0}^{1} H_{s}^{2} d s<$ $\infty$, aproximando $H$ por "integrandos simples" $H^{n}$ e defina a integral estocástica como limites em $L^{2}$ das integrais estocásticas dos integrandos aproximantes.

Uma pergunta natural é: como sabemos que o limite existe em $L^{2}$ e independente da escolha dos $H^{n} s$ ? Se $H$ é simples, podemos escrever $H$ como $\sum_{j=1}^{N} K_{j} 1_{\left[a_{j}, b_{j}\right]}(s)$, onde os $K_{j}$ s são limitados, $\mathcal{F}_{a_{j}}$-mensuráveis, e $a_{1} \leq b_{1} \leq$ $a_{2} \leq \ldots \leq b_{N}$. Então

$$
\begin{gathered}
\mathbb{E}\left[\left(\int_{0}^{1} H_{s} d X_{s}\right)^{2}\right] \\
=\mathbb{E}\left[\sum_{j=1}^{N} K_{j}^{2}\left(X_{b_{j}}-X_{a_{j}}\right)^{2}\right]+\mathbb{E}\left[2 \sum_{i<j} K_{i}\left(X_{b_{i}}-X_{a_{i}}\right) K_{j}\left(X_{b_{j}}-X_{a_{j}}\right)\right] .
\end{gathered}
$$

O segundo somando é nulo, assim temos: 


$$
\mathbb{E}\left[\left(\int_{0}^{1} H_{s} d X_{s}\right)^{2}\right]=\mathbb{E}\left[\int_{0}^{1} H_{s}^{2} d s\right]
$$

ou seja, existe uma isometria entre $\int_{0}^{1} H_{s} d X_{s}$ na norma $L^{2}(d \mathbb{P})$ e $H_{s}$ na norma $\left(\mathbb{E} \int_{0}^{1}(.)^{2} d s\right)^{1 / 2}$ em $L^{2}(d \mathbb{P} \otimes d t)$. Este resultado será usado para mostrar que o limite existe e é independente da escolha das sequências aproximantes.

Vamos voltar agora ao caso geral de martingais contínuos de modo detalhado e rigoroso. Suponha que $M_{t}$ é quadrado integrável e contínuo, assim $\sup _{t} \mathbb{E} M_{t}^{2}<\infty$. Pela desigualdade de Doob e o teorema de convergência para martingais,

$$
M_{\infty}=\lim _{t \longrightarrow \infty} M_{t}
$$

existe e $\mathbb{E} M_{\infty}^{2}<\infty$. Os integrandos $H$ que estamos considerando são predizíveis e

$$
\mathbb{E}\left[\int_{0}^{\infty} H_{s}^{2} d\langle M\rangle_{s}\right]<\infty
$$

Seja $K$ uma variável aleatória limitada $\mathcal{F}_{a}$-mensurável, e defina

$$
N_{t}=K\left(M_{t \wedge b}-M_{t \wedge a}\right)
$$

(5.1) Lema: Temos que $N_{t}$ é um martingal contínuo,

$$
\mathbb{E} N_{\infty}^{2}=\mathbb{E}\left[K^{2}\left(\langle M\rangle_{b}-\langle M\rangle_{a}\right)\right], e\langle N\rangle_{t}=\int_{0}^{t} K^{2} 1_{[a, b]}(s) d\langle M\rangle_{s} .
$$

Demonstração: A continuidade de $N_{t}$ é clara. Falta mostrar que se $s<t$, então $\mathbb{E}\left[N_{t} \mid \mathcal{F}_{s}\right]=N_{s}$. Existe alguns casos a considerar, dependendo da posição de $s$ e $t$ em relação a $a$ e $b$. Vamos analisar os casos $a<s<t<b$ e $s<a<t<b$, os demais são similares. Então

$$
\mathbb{E}\left[K\left(M_{t}-M_{a}\right) \mid \mathcal{F}_{s}\right]=K \mathbb{E}\left[M_{t}-M_{a} \mid \mathcal{F}_{s}\right]=K\left(M_{s}-M_{a}\right),
$$


no primeiro caso, como desejado. Para o segundo caso,

$$
\mathbb{E}\left[K\left(M_{t}-M_{a}\right) \mid \mathcal{F}_{s}\right]=\mathbb{E}\left[K \mathbb{E}\left[M_{t}-M_{a} \mid \mathcal{F}_{a}\right] \mid \mathcal{F}_{s}\right]=0,
$$

novamente, como precisávamos.

Para a segunda afirmação,

$$
\left.\mathbb{E} N_{\infty}^{2}=\mathbb{E}\left[K^{2} \mathbb{E}\left[\left(M_{b}-M_{a}\right)^{2} \mid \mathcal{F}_{a}\right]\right]=\mathbb{E}\left[K^{2} \mathbb{E}\left[M_{b}^{2}-M_{a}^{2}\right] \mid \mathcal{F}_{a}\right]\right]
$$

Como $M_{t}^{2}-\langle M\rangle_{t}$ é um martingal,

$$
\mathbb{E}\left[M_{b}^{2}-M_{a}^{2} \mid \mathcal{F}_{a}\right]=\mathbb{E}\left[\langle M\rangle_{b}-\langle M\rangle_{a} \mid \mathcal{F}_{a}\right] .
$$

Substituindo na equação anterior temos a segunda afirmação.

Para a terceira afirmação devemos mostrar que

$$
\begin{gathered}
\mathbb{E}\left[K^{2}\left(M_{t \wedge b}-M_{t \wedge a}\right)^{2}-K^{2}\left(\langle M\rangle_{t \wedge b}-\langle M\rangle_{t \wedge a}\right) \mid \mathcal{F}_{s}\right] \\
=K^{2}\left(M_{s \wedge b}-M_{s \wedge a}\right)^{2}-K^{2}\left(\langle M\rangle_{s \wedge b}-\langle M\rangle_{s \wedge a}\right) .
\end{gathered}
$$

Esta é análoga a primeira afirmação, trata-se novamente de considerar alguns casos.

Um processo $H_{s}$ é simples se puder ser escrito na forma $\sum_{j=1}^{J} H_{j} 1_{\left[a_{j}, b_{j}\right]}(s)$, onde para cada $j, H_{j}$ é $\mathcal{F}_{s_{j}}$-mensurável e limitado. Para um processo simples $H_{s}$, defina

$$
N_{t}=\int_{0}^{t} H_{s} d M_{s}=\sum_{j=1}^{J} H_{j}\left(M_{b_{j} \wedge t}-M_{a_{j} \wedge t}\right) .
$$


(5.2) Proposição: $S e H_{s}^{n}$ é uma sequência de processos simples tais que

$$
\mathbb{E}\left[\int_{0}^{\infty}\left(H_{s}^{n}-H_{s}^{m}\right)^{2} d\langle M\rangle_{s}\right] \longrightarrow 0
$$

quando $n, m \longrightarrow \infty$, então

$$
\mathbb{E}\left[\sup _{s<\infty}\left(N_{s}^{n}-N_{s}^{m}\right)^{2}\right] \longrightarrow 0
$$

quando $n, m \longrightarrow \infty$.

Demonstração: Se $H$ é um processo simples, pode ser reescrito como $\sum_{j=1}^{J} H_{j} 1_{\left[a_{j}, b_{j}\right]}(s)$, onde os $H_{j} s$ são limitados e $\mathcal{F}_{s_{j}}$-mensuráveis e os interva$\operatorname{los}\left[a_{j}, b_{j}\right]$ satisfazem $a_{1} \leq b_{1} \leq a_{2} \leq a_{2} \leq a_{3} \leq \ldots \leq b_{J}$. Pelo lema anterior, $N_{t}$ é um martingal, e pela desigualdade de Doob,

$$
\begin{gathered}
\mathbb{E}\left[\sup _{s<\infty} N_{s}^{2}\right] \leq c \mathbb{E}\left[N_{\infty}^{2}\right] \\
=c \mathbb{E}\left[\sum_{j=1}^{J} H_{j}^{2}\left(M_{b_{j}}-M_{a_{j}}\right)^{2}\right]+c \mathbb{E}\left[2 \sum_{i<j} H_{i} H_{j}^{2}\left(M_{b_{i}}-M_{a_{i}}\right)\left(M_{b_{j}}-M_{a_{j}}\right)\right] .
\end{gathered}
$$

Condicionando em $\mathcal{F}_{a_{j}}$ cada somando no segundo termo à direita se anula, $\log 0$

$$
\begin{gathered}
\mathbb{E}\left[H_{j}^{2}\left(M_{b_{j}}-M_{a_{j}}\right)^{2}\right]=\mathbb{E}\left[H_{j}^{2} \mathbb{E}\left[\left(M_{b_{j}}-M_{a_{j}}\right)^{2} \mid \mathcal{F}_{a_{j}}\right]\right]=\mathbb{E}\left[H_{j}^{2} \mathbb{E}\left[M_{b_{j}}^{2}-M_{a_{j}}^{2} \mid \mathcal{F}_{a_{j}}\right]\right] \\
=\mathbb{E}\left[H_{j}^{2} \mathbb{E}\left[\langle M\rangle_{b_{j}}-\langle M\rangle_{a_{j}} \mid \mathcal{F}_{a_{j}}\right]\right]=\mathbb{E}\left[H_{j}^{2}\left(\langle M\rangle_{b_{j}}-\langle M\rangle_{a_{j}}\right)\right]
\end{gathered}
$$

então

$$
\mathbb{E}\left[\sup _{s<\infty} N_{s}^{2}\right] \leq c \mathbb{E}\left[\int_{0}^{\infty} H_{s}^{2} d\langle M\rangle_{s}\right]
$$


A diferença entre dois processos simples é novamente um processo simples, assim aplicando esta estimativa para $N^{n}-N^{m}$ finalizamos a prova.

(5.3) Teorema: Suponha que $H_{s}^{n}$ são processos simples tais que

$$
\mathbb{E}\left[\int_{0}^{\infty}\left(H_{s}^{n}-H_{s}\right)^{2} d\langle M\rangle_{s}\right] \longrightarrow 0,
$$

quando $n \longrightarrow \infty$. Então $N_{s}^{n}$ converge em $L^{2}$, uniformemente sobre $s \in$ $[0, \infty)$, para um martingal continuo. O limite, o qual denotamos $\int_{0}^{t} H_{s} d M_{s}$, é independente da escolha de qual sequência $H^{n}$ utilizamos para aproximar $H_{s}$.

Demonstração: Se $H_{s}^{n}$ é como na hipótese,

$$
\mathbb{E}\left[\int_{0}^{\infty}\left(H_{s}^{n}-H_{s}^{m}\right)^{2} d\langle M\rangle_{s}\right] \longrightarrow 0,
$$

quando $n, m \longrightarrow \infty$. Pela proposição anterior temos que $N_{s}^{n}$ forma uma sequência de Cauchy em relação a norma em $L^{2}$ do supremo sobre $s$, logo esta sequência converge. Seja $N_{s}$ o limite, assim temos uma afirmação sobre convergência em $L^{2}$. Tomando uma apropriada subsequência, nós podemos dizer que com probabilidade um, $N_{s}^{n_{k}}$ converge para $N_{s}$ uniformemente sobre $s \in[0, \infty)$. Desde que cada $N_{s}^{n}$ é contínuo, isto prova que $N_{s}$ é também contínua.

Suponha $s \leq t$, pela convergência em $L^{2}$ e pela desigualdade de Jensen temos,

$$
\mathbb{E}\left[\left(\mathbb{E}\left[N_{t}^{n} \mid \mathcal{F}_{s}\right]-\mathbb{E}\left[N_{t} \mid \mathcal{F}_{s}\right]\right)^{2}\right] \leq \mathbb{E}\left[\mathbb{E}\left[\left(N_{t}^{n}-N_{t}\right)^{2} \mid \mathcal{F}_{s}\right]\right]=\mathbb{E}\left[\left(N_{t}^{n}-N_{t}\right)^{2}\right] \longrightarrow 0
$$

Visto que, $\mathbb{E}\left[N_{t}^{n} \mid \mathcal{F}_{s}\right]=N_{s}^{n}$, passando ao limite quando $n \longrightarrow \infty$, temos que $N_{t}$ é um martingal.

Finalmente, se $H^{n}, H^{m}$ são dois processos simples convergindo para $H$, a 
proposição anterior nos mostra que

$$
\mathbb{E}\left[\sup _{s<\infty}\left(N_{s}^{n}-N_{s}^{m}\right)^{2}\right] \longrightarrow 0,
$$

ou o limite é independente de qual sequência aproximante é usada.

(5.4) Corolário: Temos que

$$
\langle N\rangle_{t}=\int_{0}^{t} H_{s}^{2} d\langle M\rangle_{s}
$$

Demonstração: Esta afirmação é verdadeira pelo lema anterior quando $H$ é simples, para completar a prova basta aproximar um processo geral $H$ por processos simples.

\subsubsection{Extensões}

Vamos extender nossa definição para casos mais interessantes:

Primeiro, se $\int_{0}^{\infty} H_{s}^{2} d\langle M\rangle_{s}<\infty$, q.s. mas não necessariamente integrável, seja

$$
T_{N}=\inf \left\{t>0: \int_{0}^{T} H_{s}^{2} d\langle M\rangle_{s}>N\right\}
$$

e defina $\int_{0}^{t} H_{s} d M_{s}$ como $\int_{0}^{t} H_{s} d M_{s \wedge T_{N}}$ se $t \leq T_{N}$. Como $M_{s \wedge T_{N}}$ não aumenta em $\left[T_{N}, \infty\right)$, nós podemos usar a definição no caso integrável para definir a integral estocástica. Assim, $T_{N} \longrightarrow \infty$ quando $N \longrightarrow \infty$, nós temos assim definido $N_{t}$ para todo $t$. 
(5.5) Definições: Um processo $M_{t}$ é um martingal local se existem tempos de parada $S_{n} \longrightarrow \infty$ tais que $M_{t \wedge S_{n}}$ é um martingal de quadrado integrável para cada $n$. Se $M_{t}$ é um processo contínuo, os tempos de parada $S_{n}=\inf \left\{t:\left|M_{t}\right|>n\right\}$ servem para mostrar que é um martingal local, pois $M_{t \wedge S_{n}}$ é limitado por $n$ (note que o movimento browniano é um exemplo de martingal local que não é martingal de quadrado integrável). Para definir $\int_{0}^{t} H_{s} d M_{s}$, faça inicialmente $\int_{0}^{t} H_{s} d M_{s \wedge S_{n}}$ se $t \leq S_{n}$. A seguir tome $\langle M\rangle_{t}$ como $\langle M\rangle_{t \wedge S_{n}}$ se $t \leq S_{n}$.

Um processo é localmente de variação limitada se existem tempos de parada $R_{n}$ tais que $A_{R_{n} \wedge t}$ é de variação limitada para cada $n$ (no caso contínuo o $R_{n}$ adequado é $\left.R_{n}=\inf \left\{t: \int_{0}^{t}\left|d A_{s}\right|>n\right\}\right)$. Um semimartingal $X_{t}$ é um processo que é soma de um martingal local $M_{t}$ e um processo que é localmente de variação limitada $A_{t}$. Se $\int_{0}^{t} H_{s}^{2} d\langle M\rangle_{s}+\int_{0}^{t}\left|H_{s}\right|\left|d A_{s}\right|<\infty$ para todo $t$, definimos a integral estocástica $\int_{0}^{t} H_{s} d X_{s}=\int_{0}^{t} H_{s} d M_{s}+\int_{0}^{t} H_{s} d A_{s}$, onde a primeira integral é a integral estocástica que acabamos de discutir e a segunda é uma integral de Lebesgue-Stieltjes. Se $X_{t}$ é um semimartingal, $\langle X\rangle_{t}$ é definida como sendo $\langle M\rangle_{t}$.

\subsubsection{Fórmula de Itô}

(5.6) Teorema (Lema de Itô): Seja $X_{t}$ um semimartingal com trajetórias contínuas. Suponha que $f \in C^{2}$. Então com probabilidade um, temos

$$
f\left(X_{t}\right)=f\left(X_{0}\right)+\int_{0}^{t} f^{\prime}\left(X_{s}\right) d X_{s}+\frac{1}{2} \int_{0}^{t} f^{\prime \prime}\left(X_{s}\right) d\langle X\rangle_{s}, t \geq 0 .
$$

Demonstração: A fórmula de Itô é uma afirmação sobre trajetórias. Sabemos que $X_{t}=M_{t}+A_{t}$, onde $M$ é martingal local e $A$ é um processo que é localmente de variação limitada. Defina $T_{N}=\inf \left\{t>0:\left|M_{t}\right|>N\right.$ ou $\langle M\rangle_{t}>N$ ou $\left.\int_{0}^{t}\left|d A_{s}\right|>N\right\}$.

Como $T_{n} \longrightarrow \infty$ quando $N \longrightarrow \infty$, provando o resultado para $X_{t \wedge T_{N}}$, então temos para todo $t$. Assim, podemos assumir que $f$ é $C^{2}$ e que $f, f^{\prime}, f$ " são limitadas. 
Fixe $t_{0}>0$ e sejam $\epsilon>0, S_{0}(\epsilon)=0$, e defina

$$
\begin{gathered}
S_{i+1}(\epsilon)=\inf \left\{t>S_{i}(\epsilon):\left|M_{t}-M_{S_{i}(\epsilon)}\right|>\epsilon\right. \text { ou } \\
\left.\langle M\rangle_{t}-\langle M\rangle_{S_{i}(\epsilon)}>\epsilon \text { ou } \int_{S_{i}(\epsilon)}^{t}\left|d A_{s}\right|>\epsilon \text { ou } t-S_{i}(\epsilon)>\epsilon\right\} \wedge t_{0} .
\end{gathered}
$$

Para não carregar muita a notação vamos omitir $\epsilon$ de agora em diante.

Como estamos lidando com processos contínuos, $S_{i} \longrightarrow t_{0}$ quando $i \longrightarrow \infty$.

Note que

$$
\begin{aligned}
f\left(X_{t_{0}}\right)-f\left(X_{0}\right) & =\sum_{i=0}^{\infty}\left[f\left(X_{S_{i+1}}-f\left(X_{S_{i}}\right)\right]=\sum_{i=0}^{\infty} f^{\prime}\left(X_{S_{i}}\right)\left(X_{S_{i+1}}-X_{S_{i}}\right)\right. \\
& +\sum_{i=0}^{\infty} \frac{1}{2} f^{\prime \prime}\left(X_{S_{i}}\right)\left(X_{S_{i+1}}-X_{S_{i}}\right)^{2}+\sum_{i=0}^{\infty} R_{i},
\end{aligned}
$$

onde usamos a fórmula de Taylor e $R_{i}$ é o resto associado. Devemos mostrar que o primeiro termo à direita da equação anterior converge para o termo na forma de integral estocástica na fórmula de Itô, o segundo termo converge para o termo de variação limitada, e resto vai para zero.

Vamos primeiro olhar para o termo integral estocástica. Seja $H_{s}^{\epsilon}=f^{\prime}\left(X_{S_{i}}\right)$ se $S_{i} \leq s<S_{i+1}$. Pela continuidade de $X_{s}$ e $f^{\prime}, H_{s}^{\epsilon}$ converge limitadamente para $f^{\prime}\left(X_{s}\right)$. Então

$$
\sum_{i=0}^{\infty} f^{\prime}\left(X_{S_{i}}\right)\left(A_{S_{i+1}}-A_{S_{i}}\right)=\int_{0}^{t} H_{s}^{\epsilon} d A_{s}
$$

converge por convergência dominada usual. Note que

$$
\sum_{i=0}^{\infty} f^{\prime}\left(X_{S_{i}}\right)\left(M_{S_{i+1}}-M_{S_{i}}\right)=\int_{0}^{t} H_{s}^{\epsilon} d M_{s}
$$


Pela proposição anterior,

$$
\sum_{i=0}^{\infty} f^{\prime}\left(X_{S_{i}}\right)\left(M_{S_{i+1}}-M_{S_{i}}\right)
$$

converge para $\int_{0}^{t} f^{\prime}\left(X_{s}\right) d M_{s}$.

Temos

$$
\begin{gathered}
f^{\prime \prime}\left(X_{S_{i}}\right)\left(X_{S_{i+1}}-X_{S_{i}}\right)^{2} \\
=f^{\prime \prime}\left(X_{S_{i}}\right)\left(M_{S_{i+1}}-M_{S_{i}}\right)^{2}+2 f^{\prime \prime}\left(X_{S_{i}}\right)\left(M_{S_{i+1}}-M_{S_{i}}\right)\left(A_{S_{i+1}}-A_{S_{i}}\right) \\
+f^{\prime \prime}\left(X_{S_{i}}\right)\left(A_{S_{i+1}}-A_{S_{i}}\right)^{2} .
\end{gathered}
$$

Pelo teorema (4.22), para cada $t, V_{t}^{\epsilon}=\sum_{i: S_{i+1 \leq t}}\left(M_{S_{i+1}}-M_{S_{i}}\right)^{2}$ converge para $\langle M\rangle_{t}$ em probabilidade. Assim, se $\epsilon_{k}$ é uma sequência tendendo a zero rápido o bastante, a convergência será quase certa (veja [steele][20], página 114), $\operatorname{esup}_{\epsilon_{k}} V_{t}^{\epsilon_{k}}<\infty$.

Como $f$ " $\left(X_{s}\right)$ é um processo contínuo, $\int_{0}^{t_{0}} f^{\prime \prime}\left(X_{s}\right) d V_{s}^{\epsilon_{k}}$ converge para $\int_{0}^{t_{0}} f^{\prime \prime}\left(X_{s}\right) d\langle M\rangle_{s}$ quando $\epsilon_{k} \longrightarrow 0$. Por outro lado, como $f \in C^{2}$, dado $\delta$ podemos achar $\epsilon$ tal que $\left|f^{\prime \prime}(x)-f^{\prime \prime}(y)\right|<\delta$ se $|x-y|<\epsilon$. Portanto

$$
\sum f^{\prime \prime}\left(X_{S_{i}}\right)\left(M_{S_{i+1}}-M_{S_{i}}\right)^{2}-\int_{0}^{t} f^{\prime \prime}\left(X_{s}\right) d V_{s}^{\epsilon_{s}} \leq \delta V_{t_{0}}^{\epsilon} .
$$

Assim,

$$
\sum f^{\prime \prime}\left(X_{s}\right)\left(M_{S_{i+1}}-M_{S_{i}}\right)^{2} \longrightarrow \int_{0}^{t} f^{\prime \prime}\left(X_{s}\right) d\langle M\rangle_{s}
$$

Os demais termos são fáceis. 


$$
\begin{gathered}
\left|\sum_{i=0}^{\infty} f "\left(X_{S_{i}}\right)\left(M_{S_{i+1}}-M_{S_{i}}\right)\left(A_{S_{i+1}}-A_{S_{i}}\right)\right| \leq\|f "\|_{\infty} \epsilon \sum_{i=0}^{\infty}\left(A_{S_{i+1}}-A_{S_{i}}\right) \mid \\
\leq\|f "\|_{\infty} \in N
\end{gathered}
$$

pois estamos assumindo que $\int_{0}^{t}\left|d A_{s}\right|$ é limitada e $\left|\left(M_{S_{i+1}}-M_{S_{i}}\right)\right| \leq \epsilon$. Assim este termo vai a zero quando $\epsilon \longrightarrow 0$.

O termo $\sum f^{\prime \prime}\left(X_{S_{i}}\right)\left(A_{S_{i+1}}-A_{S_{i}}\right)^{2}$ é similar. Pelo teorema de Taylor,

$$
R_{i} \leq \eta(\epsilon)\left(X_{S_{i+1}}-X_{S_{i}}\right)^{2}
$$

onde $\eta(\epsilon) \longrightarrow 0$ quando $\epsilon \longrightarrow 0$. Como acima, $\mathbb{E}\left[\sum\left(X_{S_{i+1}}-X_{S_{i}}\right)^{2}\right]$ permanece limitado, o que mostra que $\sum R_{i} \longrightarrow 0$ em $L^{2}$.

Dados dois semimartingais contínuos $X$ e $Y$, definimos $\langle X, Y\rangle$ por polarização:

$$
\langle X, Y\rangle_{t}=\frac{1}{2}\left[\langle X+Y\rangle_{t}-\langle X\rangle_{t}-\langle Y\rangle_{t}\right]
$$

Pelo o teorema (4.22), se $M$ e $N$ são martingais, então

$$
\sum\left(M_{S_{i+1} \wedge t}-M_{S_{i} \wedge t}\right)\left(N_{S_{i+1} \wedge t}-N_{S_{i} \wedge t}\right) \longrightarrow\langle M, N\rangle_{t}
$$

Com este fato, podemos provar a versão multivariada da fórmula de Itô. 
(5.7) Corolário: Suponha que $X_{t}$ é um processo d-dimensional, onde cada componente é um semimartingal contínuo. Seja $f \in C^{2}\left(\mathbb{R}^{d}\right)$. Assim, com probabilidade um

$$
\begin{gathered}
f\left(X_{t}\right)-f\left(X_{0}\right)=\int_{0}^{t} \sum_{i=1}^{d} \frac{\partial f}{\partial x^{i}}\left(X_{s}\right) d X_{s}^{i} \\
+\frac{1}{2} \int_{0}^{t} \sum_{i, j=1}^{d} \frac{\partial^{2} f}{\partial x^{i} \partial x^{j}}\left(X_{s}\right) d\left\langle X^{i}, X^{j}\right\rangle_{s} t>0 .
\end{gathered}
$$

A seguir a fórmula de integração por partes para integrais estocásticas.

(5.8) Corolário: Se X e Y são semimartingais contínuos, então

$$
X_{t} Y_{t}=X_{0} Y_{0}+\int_{0}^{t} X_{s} d Y_{s}+\int_{0}^{t} Y_{s} d X_{s}+\langle X, Y\rangle_{t}
$$

Demonstração: Aplicando a fórmula de Itô com $f(x)=x^{2}$, obtemos $X_{t}^{2}=X_{0}^{2}+2 \int_{0}^{t} X_{s} d X_{s}+\langle X\rangle_{t}$, aplicando a fórmula de polarização obtemos o resultado.

Se $X$ é um $d$-dimensional movimento browniano, então $\left\langle X^{i}, X^{j}\right\rangle_{t}=0$ se $i \neq j$ e $\left\langle X^{i}, X^{j}\right\rangle_{t}=t$ se $i=j$, assim aplicando a fórmula de Itô para o movimento browniano temos

$$
f\left(X_{t}\right)=f\left(X_{0}\right)+\int_{0}^{t} \nabla f\left(X_{s}\right) \cdot d X_{s}+\frac{1}{2} \int_{0}^{t} \Delta f\left(X_{s}\right) d s
$$




\subsubsection{Aplicações}

Vamos começar usando a fórmula de Itô para obter as probabilidades de alcance de coroas ("annulis") para o movimento browniano $d$-dimensional, em particular obtemos a propriedades de recorrência para $d=2$ e transiência para $d \geq 3$.

(5.9) Proposição: Seja $X_{t}$ um movimento browniano d-dimensional.

(a) Se $d=2, r<|x|<R$, então

(i) $\mathbb{P}^{x}\left(T_{B(0, r)}<\tau_{B(0, R)}\right)=(\ln (R)-\ln (|x|)) /(\ln (R)-\ln (r))$;

(ii) $\mathbb{P}^{x}\left(T_{\{0\}}<\infty\right)=0$;

(iii) $\mathbb{P}^{x}\left(T_{B(0, r)}<\infty\right)=1$;

(iv) $\mathbb{P}^{x}\left(X_{t} \in B(0, r) i . v.\right)=1$.

(b) Se $d \geq 3, r<|x|<R$, então

(i) $\mathbb{P}^{x}\left(T_{B(0, r)}<\tau_{B(0, R)}\right)=\left(|x|^{2-d}-R^{2-d}\right) /\left(r^{2-d}-R^{2-d}\right) ;$

(ii) $\mathbb{P}^{x}\left(T_{\{0\}}<\infty\right)=0 ;$

(iii) $\mathbb{P}^{x}\left(T_{B(0, r)}<\infty\right)=(|x| / r)^{2-d}$.

Demonstração: Se $d=2$, seja $u(x)=-\ln (|x|)$. Se $d \geq 3$, seja $u(x)=$ $|x|^{2-d}$. Em ambos os casos temos $\nabla u=0$ em $B(0, R)-B(0, r)$ (note que $\partial|x| / \partial x^{i}=x^{i} /|x|$ pois $\left.|x|=\left(\sum_{i=1}^{d}\left(x^{i}\right)^{2}\right)^{1 / 2}\right)$. Isto implica que $M_{t}=$ $u\left(X_{t \wedge T(B(0, r))}\right)$ é um martingal. Como o movimento browniano unidimensional supera $R$ em valor absoluto com probabilidade um, então $\left|X_{t}\right| \geq\left|X_{t}^{1}\right|$ irá superar $R$ com probabilidade um. Assim, se $S=T_{B(0, r)} \wedge \tau_{B(0, R)}$, então $\mathbb{P}^{x}(S<\infty)=1$. Logo pelo corolário (4.37) obtemos (a)(i) e (b)(i).

Considere (a)(i), fazendo $r \longrightarrow 0$, obtemos $\mathbb{P}^{x}\left(T_{B(0, r)}<\tau_{B(0, R)}\right)=0$. Fazendo $R \longrightarrow 0$, obtemos (a)(ii). Se ao invés, em (a)(i), fizermos $R \longrightarrow \infty$, obtemos (a)(iii). Se fizermos $r \longrightarrow 0$ e então $R \longrightarrow 0$, em (b)(i), obtemos (b)(ii), enquanto que se fizermos $R \longrightarrow \infty$, obtemos (b)(iii). 
Para obter (a)(iv), note que para algum $s$,

$$
\mathbb{P}^{x}\left(X_{t} \in B(0, r) \text { para algum } t \geq s\right)=\mathbb{E}^{x}\left[\mathbb{P}^{X_{s}}\left(\tau_{B(0, r)<\infty}\right)\right]=1
$$

pela propriedade de Markov e (a)(iii). Ou seja, se $L=\sup \left\{t: X_{t} \in B(0, r)\right\}$, então $L \geq s$ com probabilidade um. Como $s$ é arbitrário, $L=\infty$, q.s. .

Esta proposição justifica a citação do início deste capítulo.

(5.10) Teorema: Suponha que $M_{t}$ é martingal contínuo local de trajetórias continuas com

$$
\langle M\rangle_{t} \equiv t \text { e } M_{0} \equiv 0
$$

Então $M_{t}$ é um movimento browniano.

Demonstração: Devemos mostrar que $M_{t}-M_{s}$ é independente de $\mathcal{F}_{s}$ e tem distribuição $\mathcal{N}(0, t-s)$. Considerando $M_{u}^{\prime}=M_{s+u}-M_{s}, \mathcal{F}_{u}^{\prime}=\mathcal{F}_{s+u}$, pode-se considerar apenas o caso $s=0$.

Pela fórmula de Itô,

$$
e^{i u M_{t}}-1=i u \int_{0}^{t} e^{i u M_{s}} d M_{s}-\frac{u^{2}}{2} \int_{0}^{t} e^{i u M_{s}} d s .(1)
$$

Suponha que $A \in \mathcal{F}_{0}$. Seja $T_{N}=\inf \left\{t:\left|M_{t}\right|>N\right\}$. Como o primeiro termo a direita da equação (1) é um martingal de média zero, nós temos, por parada opcional, que

$$
\mathbb{E}\left[\int_{0}^{t \wedge T_{N}} e^{i u M_{s}} d M_{s} ; A\right]=0
$$

Logo trocando $t$ por $t \wedge T_{N}$ em (2), multiplicando por $1_{A}$, tomando esperanças, 
e depois fazendo $N \longrightarrow \infty$, nós obtemos a equação

$$
J(t)=\mathbb{P}(A)-\frac{u^{2}}{2} \int_{0}^{t} J(s) d s,
$$

onde $J(s)=\mathbb{E}\left[e^{i u M_{s}} ; A\right]$.

Como $J$ é limitado, a equação (2) mostra que $J$ é contínuo, mas sendo contínuo, a equação (2) mostra também que é $C^{1}$. Assim $J^{\prime}=-u^{2} J(t) / 2$, ou $(\ln J(t))^{\prime}=-u^{2} / 2$, ou $J(t)=\mathbb{P}(A) e^{-u^{2} t / 2}$.

Esta equação pode ser reescrita como

$$
\mathbb{E}\left[e^{i u M_{t}} ; A\right]=\mathbb{P}(A) e^{-u^{2} t / 2}
$$

Tomando $A=\Omega$ temos que $M_{t}$ tem uma distribuição $\mathcal{N}(0,1)$. Aproximando $1_{B}(x)$ por combinações lineares de $e^{i u x} s$ temos

$$
\mathbb{E}\left[1_{B}\left(M_{t}\right) ; A\right]=\mathbb{P}(A) \mathbb{P}\left(M_{t} \in B\right)
$$

o que prova a independência.

Seja $\delta_{i j}$ igual a 0 se $i \neq j$ e 1 se $i=j$ (delta de Kronecker).

(5.11) Corolário: Se $X_{t}$ é um processo d-dimensional, cada coordenada é um martingal contínuo local, $X_{0} \equiv 0, e\left\langle X^{i}, X^{j}\right\rangle_{t} \equiv \delta_{i j} t$, então $X_{t}$ é um movimento browniano d-dimensional.

Demonstração: Aplicando o teorema anterior em $\sum_{j=1}^{d} \lambda_{j} X_{t}^{j}$ com $\sum_{j=1}^{d} \lambda_{j}^{2}=1$, temos:

$$
\mathbb{E}\left[e^{\sum \lambda_{j} X_{t}^{j}} ; A\right]=\mathbb{P}(A) e^{-u^{2} t / 2}
$$

Assim, fazendo $u=|v|$ e $\lambda=v /|v|$,

$$
\mathbb{E}\left[e^{i v \cdot X_{t}} ; A\right]=\mathbb{E}\left[e^{i u \lambda \cdot X_{t}} ; A\right]=e^{-|v|^{2} t / 2} \mathbb{P}(A) .
$$


(5.12) Teorema: Supondo que $M_{t}$ é um martingal contínuo com $\langle M\rangle_{t}$ estritamente crescente $e\langle M\rangle_{\infty}=\infty$. Então $M_{t}$ é uma mudança de tempo de um movimento browniano e existe um movimento unidimensional browniano $X_{t}$ tal que $M_{t}=X_{\langle M\rangle_{t}}$.

OBS.: $M_{t}$ é uma mudança de tempo para $X_{t}$ se para algum processo crescente $\tau(t)$ temos $M_{t}=X_{\tau}(t)$.

Demonstração: Definindo $\tau(u)=\inf \left\{t:\langle M\rangle_{t}>u\right\}$ e seja $X_{u}=M_{\tau(u)}$. Como $M$ tem trajetórias contínuas, então $\langle M\rangle_{t}$ também tem. Assim $\tau(u)$ é contínuo em $u$, e ainda $X$. Se $u_{1}<u_{2}$, então $\tau\left(u_{1}\right)<\tau\left(u_{2}\right)$ são tempos de parada, e por parada opcional,

$$
\mathbb{E}\left[X_{u_{2}} \mid \mathcal{F}_{\tau\left(u_{1}\right)}\right]=\mathbb{E}\left[M_{\tau_{\left(u_{2}\right)}} \mid \mathcal{F}_{\tau\left(u_{1}\right)}\right]=M_{\tau\left(u_{1}\right)}=X_{u_{1}}
$$

Portanto $X_{u}$ é um martingal com respeito às $\sigma$-álgebras $\mathcal{F}_{\tau(u)}$. Analogamente, $X_{t}^{2}-t$ é um martingal, assim $\langle X\rangle_{t} \equiv t$. Pelo teorema anterior, $X$ é um movimento browniano, e desconsiderando a mudança de tempo, $M_{t}=X_{\langle M\rangle_{t}}$.

(5.13) Definição: Dizemos que uma função $f: U \subset \mathbb{R}^{d} \longrightarrow \mathbb{R}$ é harmônica (harmônica real) se $\Delta f \equiv 0$ para todo $x \in \mathbb{R}^{d}$.

(5.14) Teorema: Seja $f: \mathbb{R}^{d} \longrightarrow \mathbb{R}$ uma função de classe $C^{2}$ em $\mathbb{R}^{2} . A$ função $f$ é harmônica se e somente se para todo movimento browniano $X_{t}$, sua imagem $f\left(X_{t}\right)$ é um martingal em $\mathbb{R}$. 
Demonstração: Dada $f$ de classe $C^{2}$ e um movimento browniano $X_{t}$ em, temos pela fórmula de Itô que

$$
\begin{aligned}
f\left(X_{t}\right)= & f\left(X_{0}\right)+\int_{0}^{t} \sum_{i=1}^{d} \frac{\partial f}{\partial x^{i}}\left(X_{s}\right) d X_{s}^{i} \\
& +\frac{1}{2} \int_{0}^{t} \sum_{i=1}^{d} \frac{\partial^{2} f}{\partial x^{i^{2}}}\left(X_{s}\right) d s
\end{aligned}
$$

já que os demais termos se anulam.

Assim, se $f$ for harmônica, o último somatório se anula, logo restam apenas termos que são martingais.

Por outro lado, suponha que $f\left(X_{t}\right)$ seja um martingal, agora, suponha por absurdo que existe $x_{0} \in \mathbb{R}^{d}$, tal que o $\Delta f\left(x_{0}\right)>0$. Sejam $U \subset$ um aberto conexo contendo $x_{0}$ tal que neste conjunto $\Delta f>0, T$ o tempo de parada dado pelo tempo de saída de $U$ pelo movimento browniano inicializado em $x_{0}$. Então

$$
\begin{aligned}
f\left(X_{t}\right)_{T}= & f\left(X_{0}\right)+\int_{0}^{T} \sum_{i=1}^{d} \frac{\partial f}{\partial x^{i}}\left(X_{s}\right) d X_{s}^{i} \\
& +\frac{1}{2} \int_{0}^{T} \Delta f\left(X_{s}\right) d s .
\end{aligned}
$$

Isto mostra que $f\left(B_{t}\right)_{T}$ não é um martingal pois a última integral é sobre um integrando estritamente positivo, absurdo, logo devemos ter $\Delta f \equiv 0$.

Note que este teorema caracteriza uma propriedade determinística ( $f$ é uma função harmônica) de modo probabilístico ( $f$ preserva a propriedade martingal do movimento browniano). 


\subsubsection{Representações Martingais}

Vamos mostrar que todo martingal adaptado a uma filtração gerada por um movimento browniano pode ser escrito como uma integral estocástica com respeito ao movimento browniano.

(5.15) Teorema: Se $Y \in L^{2}$ é $\mathcal{F}_{t}$-mensurável, existe $H_{s}$ predizível com $\mathbb{E}\left[\int_{0}^{t} H_{s}^{2} d s\right]<\infty$, tal que

$$
Y=\mathbb{E} Y+\int_{0}^{t} H_{s} d X_{s} \cdot(3)
$$

Demonstração: Existem três passos principais. Temos:

$$
\begin{gathered}
e^{u^{2} t / 2} e^{i u X_{t}}=1+\int_{0}^{t} e^{u^{2} r / 2} d\left(e^{i u X_{r}}\right)+\int_{0}^{t} e^{i u X_{r}} d\left(e^{u^{2} r / 2}\right) \\
=1+\int_{0}^{t} e^{u^{2} r / 2} i u e^{i u X_{r}} d X_{r}+\left(\frac{-u^{2}}{2}\right) \int_{0}^{t} e^{u^{2} r / 2} e^{i u X_{r}} d r+\int_{0}^{t} e^{i u X_{r}} \frac{u^{2}}{2} e^{u^{2} r / 2} d r \\
=1+\int_{0}^{t} i u e^{u^{2} r / 2} e^{i u X_{r}} d X_{r}
\end{gathered}
$$

Multiplicando pela função determinística $e^{-u^{2} t / 2}$,

$$
e^{i u X_{t}}=e^{-u^{2} t / 2}+\int_{0}^{t} i u e^{u^{2}(r-t) / 2} e^{i u X_{r}} d X_{r}
$$

Aplicando em $X_{t}^{\prime}=X_{t+s}-X_{s}$

$$
e^{i u\left(X_{t+s}-X_{s}\right)}=e^{-u^{2} t / 2}+\int_{0}^{t} i u e^{u^{2}(r-t) / 2} e^{i u X_{s+r}-X_{s}} d X_{r}
$$


ou (3) é verdade quando $Y=e^{i u\left(X_{t+s}-X_{s}\right)}$.

Para o segundo passo, suponha que temos

$$
Y_{i}=\mathbb{E} Y_{i}+\int_{0}^{t} H_{i}(r) d X_{r} i=1, \ldots, n
$$

com $H_{i}(r) H_{j}(r)=0$ se $i \neq j$. A equação (3) é válida para o produto $Y=Y_{1} \ldots Y_{n}$. Para verificar esta afirmação, vamos proceder por indução em n. Seja

$$
Y_{i}(s)=\mathbb{E}\left[Y_{i} \mid \mathcal{F}_{s}\right]=\mathbb{E} Y_{i}+\int_{0}^{s} H_{i}(r) d X_{r},
$$

$i=1,2$. Temos,

$$
\begin{gathered}
Y_{1} Y_{2}=Y_{1}(t) Y_{2}(t) \\
=Y_{1}(0) Y_{2}(0)+\int_{0}^{t} Y_{1}(r) d Y_{2}(r)+\int_{0}^{t} Y_{2}(r) d Y_{1}(r)+\left\langle Y_{1}, Y_{2}\right\rangle_{t} \\
=\mathbb{E} Y_{1} \mathbb{E} Y_{2}+\int_{0}^{t}\left[Y_{1}(r) H_{2}(r)+Y_{2}(r) H_{1}(r)\right] d X_{r}+\left\langle Y_{1}, Y_{2}\right\rangle_{t} .
\end{gathered}
$$

Note que $\left\langle Y_{1}, Y_{2}\right\rangle_{t}=\int_{0}^{t} H_{1}(r) H_{2}(r)=0$.

Para o terceiro passo, suponha que

$$
Y_{n}=\mathbb{E} Y_{n}+\int_{0}^{t} H_{n}(r) d X_{r}
$$

e $Y_{n} \longrightarrow Y$ em $L^{2}$. Então $\mathbb{E} Y_{n} \longrightarrow \mathbb{E} Y$ e

$$
\mathbb{E}\left[\int_{0}^{t}\left(H_{n}(r)-H_{m}(r)\right)^{2} d r\right]=\mathbb{E}\left[\left(\int_{0}^{t}\left(H_{n}(r)-H_{m}(r)\right) d X_{r}\right)^{2}\right]
$$




$$
\begin{gathered}
=\mathbb{E}\left[\left(\left(Y_{n}-Y_{m}\right)-\left(\mathbb{E} Y_{n}-\mathbb{E}\right)\right)^{2}\right] \\
\leq \mathbb{E}\left[\left(Y_{n}-Y_{m}\right)^{2}\right]+2\left(\mathbb{E} Y_{n}-\mathbb{E} Y_{m}\right)^{2} \longrightarrow 0
\end{gathered}
$$

quando $m, n \longrightarrow \infty$. Assim $H_{n}(r)$ forma uma sequência de Cauchy com relação à norma $\left(\mathbb{E}\left[\int_{0}^{t}(.)^{2} d r\right]\right)^{1 / 2}$. Logo existe $H$ tal que $H_{n} \longrightarrow H$ nesta norma, e $\mathbb{E}\left[\int_{0}^{t} H^{2}(r) d r\right]<\infty$. Temos

$$
\begin{gathered}
\mathbb{E}\left[\left(Y-\mathbb{E} Y-\int_{0}^{t} \mathbb{E}[H(r)] d X_{r}\right)^{2}\right] \\
=\lim _{n \rightarrow \infty} \mathbb{E}\left[\left(Y_{n}-\mathbb{E} Y_{n}-\int_{0}^{t} H_{n}(r) d X_{r}\right)^{2}\right]=0,
\end{gathered}
$$

o qual implica (3) para $Y$.

Podemos agora terminar a prova juntando os passos obtidos. Pelo primeiro e segundo passos, variáveis aleatórias da forma

$$
\prod_{j=1}^{m} e^{i u_{j}\left(X_{s_{j+1}}-X_{s_{j}}\right)}
$$

onde $s_{1} \leq \ldots \leq s_{m+1}$, satisfazem (3). Combinações lineares destas variáveis aleatórias são densas em $L^{2}$ (uma prova deste fato pode ser encontrada em [steele][20], páginas 210-211, essencialmente envolve alguns fatos de Análise Funcional em espaços de Hilbert), assim (3) também vale para todo $Y \in L^{2}$.

(5.16) Corolário: Se $M_{t}$ é um martingal com $M_{0}=0$ e $\mathbb{E} M_{t}^{2}<\infty$, então existe $H_{r}$ predizível tal que para cada s temos $M_{s}=\int_{0}^{s} H_{r} d X_{r}$, q.s. . 
Demonstração: Aplicando o teorema anterior para $Y=M_{t}$, temos que (3) vale para todo tempo $t$. Como ambos os lados de (3) são martingais, tomando esperanças condicionais em relação a $\mathcal{F}_{s}$, obtemos o corolário.

Em particular, como integrais estocásticas do movimento browniano tem caminhos contínuos, se $M_{t}$ é um martingal em relação a filtração de um movimento browniano, então $M_{t}$ tem uma versão que tem trajetórias contínuas (dois processos são versão um do outro se para cada $t$, eles são iguais quase sempre).

Seja agora $X_{t}=\left(X_{t}^{1}, \ldots, X_{t}^{d}\right)$ um movimento browniano $d$-dimensional.

(5.17) Corolário: Se $Y$ está em $L^{2}(\mathbb{P})$ e $\mathcal{F}_{t}$-mensurável, existe $H_{r}=$ $\left(H_{r}^{1}, \ldots, H_{r}^{d}\right)$ predizível com $\mathbb{E} \int_{0}^{t}\left(H_{r}^{j}\right)^{2} d r<\infty$ para cada $j$ tal que

$$
Y=\mathbb{E} Y+\int_{0}^{t} H_{r} d X_{r}
$$

Demonstração: Vamos mostrar que esta equação vale para $e^{i u . X_{t}}$, onde $u=\left(u^{1}, \ldots, u^{d}\right)$, e então seguir a demonstração do teorema (5.15). Pelo teorema $(5.15)$,

$$
Y_{j}=e^{i u_{j} X_{t}^{j}}=\mathbb{E} e^{i u_{j} X_{t}^{j}}+\int_{0}^{t} K_{r}^{j} d X_{r}^{j}
$$

Seja $H_{j}(r)=\left(0, \ldots, K_{r}^{j}, 0, \ldots, 0\right)$, se $Y_{j}(s)=\mathbb{E}\left[Y_{j} \mid \mathcal{F}_{s}\right]=\mathbb{E} Y_{j}+\int_{0}^{s} H_{j}(r) . d X_{r}$, então $\left\langle Y_{j}, Y_{k}\right\rangle_{t}=\int_{0}^{t} H_{j}(r) . H_{k}(r) d r=0$ se $j \neq k$. Pelo argumento do segundo passo do teorema (5.15), $\prod_{j=1}^{d} Y_{j}$ é uma variável aleatória que satisfaz (5.15). Como $\prod_{j=1}^{d} Y_{j}=e^{i u . X_{t}}$ obtemos nosso corolário. 


\subsubsection{Mudança de Medida e o Teorema de Suporte}

Seja $M_{t}$ um martingal contínuo positivo com $M_{0}=1$. Vamos definir uma nova medida de probabilidade $\mathbb{Q}$ fazendo $\mathbb{Q}(A)=\mathbb{E}\left[M_{t} ; A\right]$ se $A \in \mathcal{F}_{t}$, ie, a derivada de Rádon-Nikodym de $\mathbb{Q}$ com relação a $\mathbb{P}$ sobre $\mathcal{F}_{t}$ é $M_{t}$. Se $A \in \mathcal{F}_{s} \subseteq \mathcal{F}_{t}$, então $\mathbb{E}\left[M_{t} ; A\right]=\mathbb{E}\left[M_{s} ; A\right]$, como $M$ é um martingal, esta definição é consistente.

(5.18) Fórmula de Cameron-Martin-Girsanov: Se $X_{t}$ é um martingal contínuo em relação a $\mathbb{P}$, então $X_{t}-D_{t}$ é um martingal com relação $\mathbb{Q}$, onde $D_{t}=\int_{0}^{t}\left(M_{s}\right)^{-1} d\langle X, M\rangle_{s}$. A variação quadrática de $X_{t}-D_{t}$ sobre a medida $\mathbb{Q}$ é a mesma que a de $X_{t}$ sob $\mathbb{P}$.

Demonstração: Podemos assumir sem perda de generalidade que $X_{0}=0$.

Se $A \in \mathcal{F}_{s}$,

$$
\begin{gathered}
\mathbb{E}_{\mathbb{Q}}\left[X_{t} ; A\right]=\mathbb{E}_{\mathbb{P}}\left[M_{t} X_{t} ; A\right] \\
=\mathbb{E}_{\mathbb{P}}\left[\int_{0}^{t} M_{r} d X_{r} ; A\right]+\mathbb{E}_{\mathbb{P}}\left[\int_{0}^{t} X_{r} d M_{r} ; A\right]+\mathbb{E}_{\mathbb{P}}\left[\langle X, M\rangle_{t} ; A\right] \\
=\mathbb{E}_{\mathbb{P}}\left[\int_{0}^{s} M_{r} d X_{r} ; A\right]+\mathbb{E}_{\mathbb{P}}\left[\int_{0}^{s} X_{r} d M_{r} ; A\right]+\mathbb{E}_{\mathbb{P}}\left[\langle X, M\rangle_{t} ; A\right] \\
=\mathbb{E}_{\mathbb{Q}}\left[X_{s} ; A\right]+\mathbb{E}_{\mathbb{P}}\left[\langle X, M\rangle_{t}-\langle X, M\rangle_{s} ; A\right],
\end{gathered}
$$

usando a fórmula de integração por partes (corolário 5.8).

Por outro lado,

$$
\begin{gathered}
\mathbb{E}_{\mathbb{Q}}\left[D_{t}-D_{s} ; A\right]=\mathbb{E}_{\mathbb{P}}\left[\left(D_{t}-D_{s}\right) M_{t} ; A\right] \\
=\mathbb{E}_{\mathbb{P}}\left[\int_{s}^{t} M_{t} d D_{r} ; A\right]=\mathbb{E}_{\mathbb{P}}\left[\int_{s}^{t} \mathbb{E}_{\mathbb{P}}\left[M_{t} \mid \mathcal{F}_{r}\right] d D_{r} ; A\right]
\end{gathered}
$$




$$
\begin{gathered}
=\mathbb{E}_{\mathbb{P}}\left[\int_{s}^{t} M_{r} d D_{r} ; A\right]=\mathbb{E}_{\mathbb{P}}\left[\int_{s}^{t} d\langle X, M\rangle_{r} ; A\right] \\
=\mathbb{E}_{\mathbb{P}}\left[\langle X, M\rangle_{t}-\langle X, M\rangle_{s} ; A\right] .
\end{gathered}
$$

Isto mostra que $X_{t}-D_{t}$ é um martingal com relação a $\mathbb{Q}$. A parte relativa à variação quadrática pode ser mostrada do mesmo modo.

Vamos usar este resultado para provar o teorema de suporte.

(5.19)Proposição: $S e X_{t}$ é um movimento browniano d-dimensional, $\epsilon>$ 0 e $t>0$, então

$$
\mathbb{P}^{0}\left(\sup _{s \leq t}\left|X_{s}\right|<\epsilon\right)>0 .
$$

Demonstração: Vamos mostrar este resultado para o caso unidimensional, o caso geral segue de

$$
\begin{gathered}
\mathbb{P}^{0}\left(\sup _{s \leq t}\left|X_{s}\right|<\epsilon\right) \geq \mathbb{P}^{0}\left(\sup _{s \leq t}\left|X_{s}^{i}\right|<\epsilon / \sqrt{d}, i=1, \ldots, d\right) \\
\geq\left(\mathbb{P}^{0}\left(\sup _{s \leq t}\left|X_{s}^{1}\right|<\epsilon / \sqrt{d}\right)\right)^{d}>0 .
\end{gathered}
$$

Basta mostrar no caso unidimensional, que

$$
\mathbb{P}^{0}\left(\sup _{s \leq u}\left|X_{s}\right|<1\right)>0,(*)
$$


nós obtemos o resultado desejado trocando $u$ por $t / \sqrt{\epsilon}$ e usando a propriedade de escala.

Note que se $|x| \leq 1 / 4$ e $n$ é suficientemente grande, então $\mathbb{P}^{x}\left(\left|X_{1 / n}\right| \leq 1 / 4\right) \geq$ $1 / 4$, isto ocorre porque $\mathbb{P}^{x}\left(\left|X_{1 / n}-X_{0}\right|<1 / 4 e X_{1 / n}<X_{0}\right) \longrightarrow 1 / 2$ quando $n \longrightarrow \infty$ pela simetria da normal. Pela proposição (3.12),

$$
\sup _{|x| \leq 1 / 4} \mathbb{P}^{x}\left(\sup _{s \leq 1 / n}\left|X_{s}-X_{0}\right|>1 / 4\right) \leq 2 e^{-n / 32} .
$$

Mais ainda,

$$
\inf _{|x| \leq 1 / 4} \mathbb{P}^{x}\left(\sup _{s \leq 1 / n}\left|X_{s}\right|<1 / 2,\left|X_{1 / n}\right| \leq 1 / 4\right) \geq(1 / 4)-2 e^{-n / 32} .
$$

Tomando $n$ suficientemente grande, isto será maior do que 1/8.

Seja $I_{m}=\left(\sup _{s \leq 1 / n}\left|X_{s+m / n}-X_{m / n}\right|<1 / 2,\left|X_{(m+1) / n}\right| \leq 1 / 4\right)$. Pela propriedade de Markov,

$$
\mathbb{P}\left(I_{1} \cap I_{2} \cap \ldots \cap I_{m}\right) \geq(1 / 8)^{m}
$$

Note que $\left(\sup _{s \leq m / n}\left|X_{s}\right|<1\right) \supseteq I_{1} \cap I_{2} \cap \ldots \cap I_{m}$, assim tomando $m>n u$ provamos $(*)$ e assim a proposição.

Como último resultado deste capítulo, vamos enunciar o teorema de suporte para o movimento browniano:

(5.20) Teorema de Suporte: Se $\psi:[0, t] \longrightarrow \mathbb{R}^{d}$ é contínua, $\epsilon>0$, e $x=\psi(0)$, então

$$
\mathbb{P}^{x}\left(\sup _{s \leq t}\left|X_{s}-\psi(s)\right|<\epsilon\right)>c
$$


onde c pode ser tomado dependendo somente de $t, \epsilon$, e o módulo de continuidade de $\psi$.

Demonstração: Vamos tomar $\psi_{1}$ tal que $\psi_{1}$ tem derivada limitada e tal que $\sup _{s \leq t}\left|\psi(s)-\psi_{1}(s)\right|<\epsilon / 2$. Podemos escolher $\psi_{1}$ de modo que $\left\|\psi_{1}^{\prime}\right\|_{\infty}$ depende somente de $t, \epsilon$, e o módulo de continuidade de $\psi$. Vamos mostrar que

$$
\mathbb{P}^{x}\left(\sup _{s \leq t}\left|X_{s}-\psi_{1}(s)\right|<\epsilon / 2\right)>c,(* *)
$$

onde $c$ depende somente de $t, \epsilon$, e $\left\|\psi_{1}^{\prime}\right\|_{\infty}$, isto nos dá o teorema.

Defina $\mathbb{Q}$ por $d \mathbb{Q} / d \mathbb{P}=M_{t}$, onde

$$
M_{t}=\exp \left(\int_{0}^{t} \psi_{1}^{\prime}(s) \cdot d X_{s}-(1 / 2) \int_{0}^{t}\left|\psi_{1}^{\prime}(s)\right|^{2} d s\right)
$$

Note que

$$
\left\langle X, \int_{0}^{t} \psi_{1}^{\prime}(s) \cdot d X_{s}\right\rangle_{t}=\int_{0}^{t} \psi_{1}^{\prime}(s) d s=\psi_{1}(t)-\psi_{1}(0) .
$$

Assim, $X_{t}-\psi_{1}(t)$ é um processo, onde cada uma das componentes sob $\mathbb{Q}$ é um martingal contínuo, e a variação quadrática é a mesma que a de $X$ sob $\mathbb{P}$. Logo pelo corolário $(5.11)$, sob $\mathbb{Q}, X(t)-\psi_{1}(t)$ é um movimento browniano iniciando em 0 . Pela proposição anterior, $\mathbb{Q}(A) \geq c$, onde $A=\left(\sup _{s \leq t}\left|X(s)-\psi_{1}(s)\right|<\epsilon / 2\right)$, e $c$ depende somente de $\epsilon$ e $t$. Como $\left|\psi_{1}^{\prime}\right|$ é limitada, $\mathbb{E} M_{t}^{2}<\infty$ e

$$
c \leq \mathbb{Q}(A)=\int_{A} M_{t} d \mathbb{P} \leq\left(\mathbb{E} M_{t}^{2}\right)^{1 / 2}(\mathbb{P}(A))^{1 / 2},
$$

o que prova $(* *)$.

Este teorema junto com a propriedade de invariância sob escala do movimento browniano justificam a citação do próximo capítulo (capítulo 6). 


\section{Cálculo Estocástico Complexo}

If you run Brownian motion in two dimensions for a positive amount of time, it will write your name.

Wilfrid Kendall

Neste capítulo vamos retomar algumas construções do capítulo anterior no caso complexo. A conexão entre o movimento browniano bidimensional e a Análise Complexa vem através de um resultado de Lévy que diz que uma função analítica composta com um movimento browniano bidimensional é uma mudança de tempo de outro movimento browniano.

\subsection{Cálculo Estocástico Complexo}

Sejam $\mathbb{D}$ o disco unitário $\{z:|z|<1\}$, e $\mathbb{H}$ o semi-plano $\{z: \Im z>0\}$. Vamos escrever $\tau$ para $\tau_{\mathbb{D}}$ e $\tau$ para $\tau_{B(0, r)}$.

(6.1) Definição: $O$ movimento browniano complexo padrão $Z_{t}=$ $X_{t}+i Y_{t}=\left(X_{t}, Y_{t}\right)$ é um processo estocástico em $\mathbb{C}$, iniciando em zero e adaptado ao espaço de probabilidade $\left(\Omega, \mathcal{F}, \mathcal{F}_{t}, \mathbb{P}\right)$, onde $X_{t}=\Re Z_{t}$ e $Y_{t}=\Im Z_{t}$, são movimentos brownianos unidimensionais.

Note que estamos tomando o movimento browniano bidimensional real como um movimento browniano complexo unidimensional. Assim, um processo $Z_{t}$ é um movimento browniano complexo padrão se e somente $\left(X_{t}, Y_{t}\right)$ é um movimento browniano bidimensional padrão sobre o plano $(x, y)$ (ou seja, $\left.\mathbb{R}^{2}\right)$.

(6.2) Definição: Um martingal complexo é um processo $M_{t}$ sobre $\mathbb{C}$ (ou $\mathbb{C}^{d}$ ), que é um martingal vetorial (multivariado) quando $\mathbb{C}$ (ou $\mathbb{C}^{d}$ ) é visto como espaço vetorial real.

Note que se $M_{t}$ é um martingal complexo, então seu conjugado $\bar{M}_{t}$ também 
é um martingal complexo. Quanto à definição do processo $\langle M, N\rangle$ devemos fazer uma escolha, tomá-lo bilinear ou sesquilinear, nesta dissertação escolheremos a bilinearidade como convenção.

(6.3) Definição: Sejam $M$ e $N$ martingais complexos contínuos de quadrado integráveis, então $\langle M, N\rangle$ é o único processo complexo contínuo adaptado e de variação limitada tal que $M_{t} N_{t}-\langle M, N\rangle_{t}$ é um martingal.

(6.4) Lema: $S e M_{t}=R_{t}+i S_{t}, N_{t}=T_{t}+i U_{t}$, onde $R_{t}, S_{t}, T_{t}$ e $U_{t}$ são martingais reais então

$$
\langle M, N\rangle_{t}=\left(\langle R, T\rangle_{t}-\langle S, U\rangle_{t}\right)+i\left(\langle R, T\rangle_{t}+\langle U, S\rangle_{t}\right)
$$

A demonstração desta proposição segue após algumas contas rotineiras.

(6.5) Definição: Um martingal complexo contínuo de quadrado integrável é um martingal conforme se $\langle M, M\rangle_{t} \equiv 0$.

Note que um martingal $M_{t}$ é conforme se e somente se seu quadrado $M_{t}^{2}$ é também um martingal conforme.

(6.6) Lema: O movimento browniano complexo é um martingal conforme.

Demonstração: Seja $Z_{t}=X_{t}+i Y_{t}$ um movimento browniano complexo, onde $X_{t}=\Re Z_{t}$ e $Y_{t}=\Im Z_{t}$, são movimentos brownianos unidimensionais. Assim,

$$
\langle Z, Z\rangle_{t}=\langle X, X\rangle_{t}-\langle Y, Y\rangle_{t}+2 i\langle X, Y\rangle_{t}=1 / 2(t-t)+0=0
$$


O processo $\langle Z, \bar{Z}\rangle_{t}$ faz o papel de variação quadrática para martingais conformes.

Podemos agora reescrever o lema de Itô para o caso bidimensional na forma complexa:

(6.7) Teorema(Lema de Itô Complexo): Suponha que $Z$ é martingal complexo contínuo e que $f$ é uma função de classe $C^{2}$, então

$$
\begin{gathered}
f\left(Z_{t}\right)-f\left(Z_{0}\right)=\int_{0}^{t} \partial f\left(Z_{s}\right) d Z_{s}+\int_{0}^{t} \bar{\partial} f\left(Z_{s}\right) d \bar{Z}_{s} \\
+\int_{0}^{t} \partial \partial f\left(Z_{s}\right) d\langle Z, Z\rangle_{s}+\int_{0}^{t} \overline{\partial \partial} f\left(Z_{s}\right) d\langle\bar{Z}, \bar{Z}\rangle_{s} \\
+2 \int_{0}^{t} \partial \bar{\partial} f\left(Z_{s}\right) d\langle Z, \bar{Z}\rangle_{s} .
\end{gathered}
$$

Os seguintes corolários são imediatos:

(6.8) Corolário: $S e Z_{t}$ é um martingal conforme nós temos

$$
f\left(Z_{t}\right)-f\left(Z_{0}\right)=\int_{0}^{t} \partial f\left(Z_{s}\right) d Z_{s}+\int_{0}^{t} \bar{\partial} f\left(Z_{s}\right) d \bar{Z}_{s}+2 \int_{0}^{t} \partial \bar{\partial} f\left(Z_{s}\right) d\langle Z, \bar{Z}\rangle_{s} .
$$

(6.9) Corolário: $S e Z_{t}$ é um martingal conforme e $f$ é harmônica então $f\left(Z_{t}\right)$ é um martingal e temos

$$
f\left(Z_{t}\right)-f\left(Z_{0}\right)=\int_{0}^{t} \partial f\left(Z_{s}\right) d Z_{s}+\int_{0}^{t} \bar{\partial} f\left(Z_{s}\right) d \bar{Z}_{s} .
$$


(6.10) Corolário: $S e Z_{t}$ é um martingal conforme e $f$ é analítica então $f\left(Z_{t}\right)$ é um martingal e temos

$$
f\left(Z_{t}\right)-f\left(Z_{0}\right)=\int_{0}^{t} f^{\prime}\left(Z_{s}\right) d Z_{s}
$$

\subsection{Invariância Conforme}

Vamos separar o teorema de Lévy em duas partes, inicialmente suponha que $f$ é inteira e não constante.

(6.11) Teorema de Lévy (invariância conforme): Sejam

$$
A_{s}=\int_{0}^{s}\left|f^{\prime}\left(Z_{r}\right)\right|^{2} d r \text { e } \sigma_{t}=\inf \left\{s: A_{s} \geq t\right\}
$$

Então $f\left(Z_{\sigma_{t}}\right)$ é um movimento browniano bidimensional.

Demonstração: Se uma função é analítica em um domínio e seus zeros têm um ponto de acumulação no domínio, então a função deve ser identicamente nula (veja [rudin][16]). Assim, como $f$ é não constante, $f^{\prime}$ tem no máximo um número contável de zeros; como o movimento browniano bidimensional não atinge um conjunto contável (pela proposição 5.9 (a)(ii), a probabilidade de alcance é zero), $A_{s}$ é estritamente crescente. Logo $\sigma_{t}$ é contínuo em $t$, q.s., e $f\left(Z_{\sigma_{t}}\right)$ é um processo contínuo.

Antes de continuar, vamos provar o seguinte lema:

(6.12) Lema: Se $f$ é inteira, não identicamente nula, então $A_{s} \longrightarrow \infty$, q.s., quando $s \longrightarrow \infty$. 
Demonstração: Se $f$ é constante não nula, então a conclusão é trivialmente verdadeira. Se $f$ é não constante então pelo teorema da aplicação aberta, podemos assumir que existe um disco $\mathbb{D}\left(z_{0}, \rho\right)$ e um $\delta>0$ tais que $|f(z)| \geq \delta>0, \forall z \in \mathbb{D}\left(z_{0}, \rho\right)$. Agora, sejam $\tau_{0}=0$, para $j=1,2, \ldots$,

$$
\begin{gathered}
\sigma_{j}=\inf \left\{s>\tau_{j-1}: Z_{s} \in \mathbb{D}\left(z_{0}, \rho / 2\right)\right\} e \\
\tau_{j}=\inf \left\{s>\sigma_{j}: Z_{s} \neq \mathbb{D}\left(z_{0}, \rho / 2\right)\right\} .
\end{gathered}
$$

Claramente, $\left\{\tau_{j}-\sigma_{j}\right\}$ é uma sequência de variáveis aleatórias positivas independentes e identicamente distribuídas (i.i.d.) e temos ainda que $\left|f\left(Z_{s}\right)\right| \geq$ $\delta, \sigma_{j} \leq s \leq \tau_{j}, \forall j \geq 1$. Logo

$$
\int_{0}^{s}\left|f\left(Z_{r}\right)\right|^{2} d r \geq \delta \sum_{j=1}^{\infty}\left(\tau_{j} \wedge t-\sigma_{j} \wedge t\right) \longrightarrow \infty
$$

q.s., quando $s \longrightarrow \infty$.

Assim, $A_{s} \longrightarrow \infty$, q.s., quando $s \longrightarrow \infty$.

Suponha $f=u+i v$. Pela fórmula de Itô,

$$
\begin{gathered}
u\left(Z_{t}\right)=u\left(Z_{0}\right)+\int_{0}^{t} \nabla u\left(Z_{s}\right) d Z_{s} \quad e \\
v\left(Z_{t}\right)=v\left(Z_{0}\right)+\int_{0}^{t} \nabla v\left(Z_{s}\right) d Z_{s}
\end{gathered}
$$

Em particular, $u\left(Z_{t}\right)$ e $v\left(Z_{t}\right)$ são martingais. Pelas equações de CauchyRiemann nós temos $|\nabla u(z)|^{2}=|\nabla v(z)|^{2}$ e $\nabla u(z) . \nabla v(z)=0$ para todo $z$. $\operatorname{Assim}\langle u(Z)\rangle_{t}=\langle v(Z)\rangle_{t}=A_{t}$ e $\langle u(Z), v(Z)\rangle_{t}=0$.

Portanto, $f\left(Z_{\sigma}\right)$ é um processo contínuo bidimensional, sendo cada componente um martingal, $\left\langle u\left(Z_{\sigma .}\right)\right\rangle_{t}=\left\langle v\left(Z_{\sigma .}\right)\right\rangle_{t}=t$, e $\left\langle u\left(Z_{\sigma .}\right), v\left(Z_{\sigma .}\right)\right\rangle_{t}=0$. Pelo 
teorema (5.11), $f\left(Z_{\sigma_{t}}\right)$ é um movimento browniano bidimensional.

A seguir a versão do teorema de Lévy para funções analíticas em um domínio D.

(6.13) Teorema: Suponha que $f$ é analítica em um dominio D. Seja $A_{s}$ e $\sigma_{t}$ como no teorema anterior. Se $F_{T}=f\left(Z\left(\sigma_{t \wedge \tau_{D}}\right)\right)$, então $F_{t \wedge \tau(f(D))}$ é um movimento browniano bidimensional parado sobre a borda de $f(D)$.

Demonstração: Como na prova do teorema anterior, $F_{t}$ é um martingal de tempo contínuo com $\langle\Re F\rangle_{t}=\langle\Im F\rangle_{t}=t \wedge \tau_{D}$ e $\langle\Re F, \Im F\rangle_{t}=0$. Seja $W_{t}$ um movimento browniano bidimensional iniciando em zero e independente de $Z_{t}$ e defina $F_{t}^{\prime}=F_{t}$ se $t \leq \tau_{D}$ e $F_{t}^{\prime}=F_{t}$ se $F_{t}^{\prime}=F_{\tau_{D}}+W_{t-\tau_{D}}>\tau_{D}$.

É fácil ver que $\left\langle\Re F^{\prime}\right\rangle_{t}=\left\langle\Im F^{\prime}\right\rangle_{t}=t$ e $\left\langle\Re F^{\prime}, \Im F^{\prime}\right\rangle_{t}=0$, e assim $F_{t}^{\prime}$ é um movimento browniano bidimensional. Não é difícil ver que $f\left(Z_{t}\right)$ primeiro alcança $\partial f(D)$ antes ou ao mesmo tempo que $Z_{t}$ primeiro alcança $\partial D$. Assim $F_{t \wedge \tau(f(D))}$ é um movimento browniano bidimensional parado sobre $\partial f(D)$.

Se $f$ é injetora, então o tempo de alcance de $\partial f(D)$ por $f\left(Z_{t}\right)$ é igual ao tempo de alcance de $\partial D$ por $Z_{t}$.

Antes de passar às aplicações do Teorema de Lévy, parece bastante natural se perguntar se ele não se generaliza para dimensões $d \geq 3$, a resposta é não, vamos ver porque.

Seja $F_{t}^{i}$ a $i$-ésima componente de $f\left(B_{t}\right)$. Se $f\left(B_{t}\right)$ é uma mudança de tempo do movimento browniano, devemos ter $\left\langle F^{i}, F^{j}\right\rangle \equiv \delta_{i j} A_{t}$, assim segue da fórmula de Itô que se $f_{i}$ é a $i$-ésima componente de $f$, então

$\nabla f_{i} \cdot \nabla f_{j}=0$ se $i \neq j$ e

$\left|\nabla f_{i}\right|^{2}$ é independente de $i$

As duas últimas condições implicam que $f$ é conforme (preserva ângulos), 
logo por um teorema de Liouville (veja [spivak][18]) temos que $f$ deve ser uma composição de funções da seguinte lista:

(i) translação: $x \longrightarrow x+y$;

(ii) multiplicação por uma constante (homotetia): $x \longrightarrow c x$;

(iii) transformação ortogonal: $x \longrightarrow A x$ onde $A$ é uma matriz ortogonal;

(iv) inversão: $J(x)=x /|x|^{2}$.

As funções $f$ que podem ser formadas pela composição de funções da lista anterior são chamadas de Transformações de Kelvin. As três primeiras transformações claramente levam o movimento browniano em uma mudança de tempo dele mesmo, porém se $d \geq 3$, a última transformação não.

Quando $d \geq 3,\left|B_{t}\right| \longrightarrow \infty$ quando $t \longrightarrow \infty$, assim $J\left(B_{t}\right) \longrightarrow 0$, algo que é impossível para uma mudança de tempo do movimento browniano em $d \geq 3$, pois neste caso $\mathbb{P}\left(B_{t}=0\right.$ para algum $\left.t>0\right)=0$ e, como mencionado, $\left|B_{t}\right| \longrightarrow \infty$ quando $t \longrightarrow \infty$.

\subsection{Aplicações}

Vamos finalmente mostrar alguns teoremas clássicos de Análise Complexa através do Cálculo Estocástico.

\subsubsection{Alguns Teoremas Interessantes}

(6.14) Teorema Fundamental da Álgebra: Seja $P(z)$ um polinômio não constante em z. Então existe pelo menos um $z_{0}$ tal que $P\left(z_{0}\right)=0$, ou seja, todo polinômio complexo não constante possui pelo menos uma raiz.

Demonstração: Se $P(z)=a_{n} z^{n}+a_{n-1} z^{n-1}+\ldots+a_{0}$ com $a_{n} \neq 0$, então

$$
|P(z)|=\left|a_{n}\right||z|^{n}\left|1+\frac{a_{n-1}}{a_{n}} z^{-1}+\ldots+\frac{a_{0}}{a_{n}} z^{-n}\right| \longrightarrow \infty,
$$

quando $|z| \longrightarrow \infty$.

Seja $R$ tal que $|P(z)| \geq 1$ se $|z| \geq R$. 


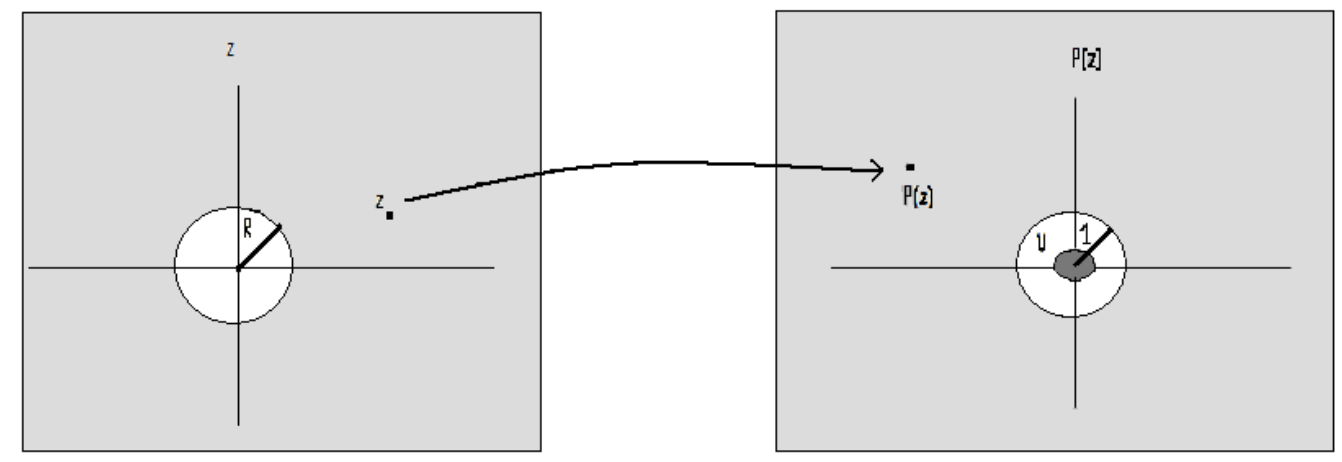

Figura 1: Planos complexos domínio e imagem de $P(z)$

Suponha que $P(z)$ nunca se anule. Como $P(\overline{B(0, R)})$ é compacto, e logo fechado, existe uma vizinhança $V$ de 0 que é disjunta dele. Escolhendo $V$ de modo que seu diâmetro seja menor do que $1 / 2, V \cap P(\mathbb{C})=\emptyset$ (acompanhe a figura 1, para uma melhor compreensão da situação descrita).

Entretanto, $P\left(Z_{t}\right)$ é uma mudança de tempo de um movimento browniano bidimensional. Como $P$ é não constante, temos que $\langle P(Z)\rangle_{t} \longrightarrow \infty$ quando $t \longrightarrow \infty$. Isto significa que $P\left(Z_{t}\right)$ alcança toda vizinhança infinitas vezes; em particular toca $V$, uma contradição para $V \cap P(\mathbb{C})=\emptyset$.

(6.15) Teorema de Liouville: Toda função f inteira não constante é não limitada.

Demonstração: Pela propriedade de recorrência para o movimento browniano bidimensional (proposição 5.9) temos que com probabilidade um, quase toda trajetória de $f\left(Z_{t}\right)$ é densa em $\mathbb{C}$.

(6.16) Princípio do Máximo Módulo: Se D é um domínio limitado e $f$ é analítica em $D$ e contínua em $\bar{D}$, então $\sup _{\bar{D}}|f|=\sup _{\partial D}|f|$. 
Demonstração: Se $f=u+i v$ onde $u$ e $v$ são harmônicas, então $|f|^{2}=$ $u^{2}+v^{2}$ é subharmônica, como $\delta\left(u^{2}\right)=2|\nabla u|^{2} \geq 0$ e mesmo vale para $\delta\left(v^{2}\right)$. Assim $|f(z)|^{2} \leq \mathbb{E}^{z} \mid f\left(\left.Z_{\tau_{D}}\right|^{2} \leq \sup _{\partial D}|f|^{2}\right.$ para $z \in D$. Agora basta tomar raízes quadradas para obter o resultado.

\subsection{2 "Pequeno"Teorema de Picard}

Dadas duas curvas fechadas $\gamma_{1}$ e $\gamma_{2}$ dizemos que elas são homotópicas em um domínio $D$ se $\gamma_{1}$ pode ser deformada continuamente em $\gamma_{2}$.

De modo mais formal, suponha que $\gamma_{1}$ e $\gamma_{2}$ são funções contínuas de $[0,1]$ em um domínio $D$ com $\gamma_{i}(0)=\gamma_{i}(1), i=1,2$. Dizemos que $\gamma_{1}$ e $\gamma_{2}$ são homotópicas se existe uma função $F$ de $[0,1]^{2}$ em $D$ tal que $F$ é contínua, $F(s, 0)=\gamma_{1}(s)$ para todo $s \in[0,1], F(s, 1)=\gamma_{2}(s)$ para todo $s \in[0,1]$, e $F(0, t)=F(1, t)$ para todo $t \in[0,1]$. O conjunto de curvas homotópicas umas às outras forma claramente uma classe de equivalência. Além disso, se $\gamma_{1}$ e $\gamma_{2}$ são homotópicas entre si e $f$ é contínua, então $f\left(\gamma_{1}\right)$ e $f\left(\gamma_{2}\right)$ são homotópicas entre si. Em um domínio simplesmente conexo cada curva fechada é homotópica a curva $\gamma_{0}$ formada por um único ponto no domínio.

(6.17) "Pequeno"Teorema de Picard: Se f é uma função inteira e se existirem dois números complexos que não estão na imagem de $f$, então $f$ é constante, ou seja, a imagem de $f$ omite no máximo um ponto se $f$ é uma função inteira não constante.

Demonstração: A prova original deste resultado segundo métodos de Cálculo Estocástico se deve a [davis2][5], aqui seguiremos uma variante devida a $[$ bass] $[2]$.

Seja $f$ inteira não constante, suponha por absurdo que $f(\mathbb{C})$ omite mais de um ponto de $\mathbb{C}$. Podemos sem perda de generalidade supor que dois dos pontos omitidos são $-a$ e $b$, onde $a$ e $b$ são reais positivos, e que $f(0)=0$. Como $f$ é inteira, $\left|f^{\prime}\right|>0$ exceto para um conjunto contável, e pela recorrência do movimento browniano, $\int_{0}^{t}\left|f^{\prime}\left(Z_{s}\right)\right|^{2} d s \longrightarrow \infty$ quando $t \longrightarrow \infty$. Assim $f\left(Z_{t}\right)$ é um movimento browniano a menos de uma mudança de tempo (não morto ou parado). 


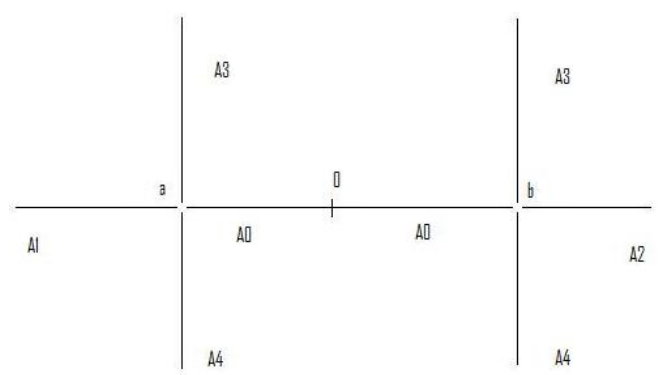

Figura 2: Plano complexo com conjuntos $A_{i}$

Seja $\epsilon>0$ suficientemente pequeno tal que se $|z|<\epsilon, f(z)$ pode ser conectado a 0 por uma curva passando por $f(\mathbb{C}) \cap B(0,(a \wedge b) / 2)$. Para infinitos $t, Z_{t} \in B(0, \epsilon)$. Seja $L_{t}$ o segmento de reta conectando $Z_{t}$ a 0 . A curva consistindo de adicionar o segmento de reta $L_{t}$ ao final de $Z_{t}$ é uma curva fechada homotópica a um único ponto, 0 . Logo a curva consistindo de adicionar $f\left(L_{t}\right)$ ao final de $f\left(Z_{t}\right)$ deve ser também homotópica a um único ponto.

Vamos mostrar agora que em $L_{t}$ com probabilidade um, existe $t_{0}$ (dependendo de $\omega$ ) tal que se $t>t_{0}$, a curva formada pela adição de $f\left(L_{t}\right)$ ao final de $f\left(Z_{t}\right)$ é não homotópica a um único ponto $\left(^{*}\right)$.

Sejam

$$
\begin{gathered}
A_{0}=\{-a<x<b, y=0\}, A_{1}=\{x<-a, y=0\} \\
A_{2}=\{x>b, y=0\}, A_{3}=\{x=-a \text { ou } x=b, y>0\} e \\
A_{4}=\{x=- \text { a ou } x=b, y<0\} .
\end{gathered}
$$

Sejam $T_{1}=0, b_{i} \in\{0,1,2,3,4\}$ tais que $f\left(Z_{T_{i}}\right) \in A_{b_{i}}$, e

$$
T_{i+1}=\inf \left\{t>T_{i}: f\left(Z_{t}\right) \in \cup_{j=0}^{4} A_{j}-A_{b_{i}}\right\} .
$$

Assim os $T_{i} s$ são os tempos para atingir um dos conjuntos $A_{j}$ diferentes da última passagem.

Seja $0 b_{1} b_{2} \ldots b_{n}$ a sequência formada, e então seja a sequência reduzida construída como segue: 
(i) Se nossa sequência termina em 030 ou 040, apague as duas últimas entradas;

(ii) Se a última entrada é a mesma que a antepenúltima e a penúltima não é 0, apague as duas últimas entradas (assim, ...0424 torna-se ...04, mas ...3404 não se torna ...34);

(iii) Se a sequência termina em um 0 e tem a forma $\ldots 0 b_{i_{1}} \ldots b_{i_{j}} 0 b_{i_{j}} \ldots b_{i_{1}} 0$, elimine tudo a partir do antepenúltimo 0 até o último (assim ...0423140413240 torna-se ...0).

Aplicando as regras (i), (ii) e (iii) até a sequência não puder ser mais reduzida, obtemos a sequência completamente reduzida (acompanhar os passos do algoritmo através da figura 2 , fornece uma maior intuição sobre o processo).

A sequência completamente reduzida descreve a classe de homotopia para $f\left(Z_{t}\right)$ com $f\left(L_{t}\right)$ adicionada sobre $Z_{t} \in B(0, \epsilon)$, e para esta curva ser homotópica a um ponto, é necessário que a sequência reduzida consista de um única entrada 0. Assim para provar $\left(^{*}\right)$, é suficiente provar que o número de $0 s$ em uma sequência reduzida tende a $\infty$, q.s. . Seja um bloco uma porção de uma sequência reduzida iniciando e terminando com um 0 e sem zeros no meio.

Suponha que o último bloco na sequência reduzida é ...03140. Para reduzir o número de zeros, o próximo bloco deve ser 04130. Por simetria na reta $y=0$, temos 03140, o que aumenta o número de zeros por um. Além disso, pelo teorema de suporte, existe uma probabilidade positiva que o próximo bloco seja 034240 ou 042430, cada uma das quais aumenta o número de zeros. Um argumento similar de simetria pode ser dado não importa o que o último bloco é, e assim existe $\delta>0$ tal que

$$
\begin{gathered}
\mathbb{P}(\text { numero de zeros aumenta em um }) \geq 1 / 2+\delta \text { e } \\
\mathbb{P}(\text { numero de zeros diminui em um }) \leq 1 / 2-\delta
\end{gathered}
$$

Logo o número de $0 s$ tende ao infinito, q.s..

Vamos retornar à curva consistindo de adicionar o segmento de reta $f\left(L_{t}\right)$ ao final de $f\left(Z_{t}\right)$. Para $t$ suficientemente grande, esta curva é não homotópica a um único ponto, uma contradição, o que prova o teorema. 


\section{Conclusão}

It is not knowledge, but the act of learning, not possession but the act of getting there, which grants the greatest enjoyment. When I have clarified and exhausted a subject, then I turn away from it, in order to go into darkness again; the never-satisfied man is so strange if he has completed a structure, then it is not in order to dwell in it peacefully, but in order to begin another. I imagine the world conqueror must feel thus, who, after one kingdom is scarcely conquered, stretches out his arms for others.

Johann Carl Friedrich Gauss (1777-1855)

Na relação frutífera entre Análise e Probabilidade, normalmente o fluxo de aplicações se dá no uso da Análise como ferramenta poderosa para estudar a Teoria da Probabilidade. Neste texto procuramos explorar um pouco desta relação no sentido oposto, em particular na aplicação de Probabilidade (Cálculo Estocástico) para obter resultados clássicos em Análise Complexa.

Aqui apresentamos apenas uma pequena amostra das muitas possíveis aplicações, mais desenvolvimentos podem ser encontrados em [bass][2] e [durrett1][6].

Como sabemos, o "pequeno"teorema de Picard é generalizado pela Teoria de Nevanlinna, assim cabe a pergunta: Podemos usar o Cálculo Estocástico para provar os teoremas de Nevanlinna?

A resposta é sim, e o leitor deste texto poderá encontrar detalhes em [atsuji][1] e [carne][3]. Uma prova do "grande"teorema de Picard pode ser encontrada em [davis2][5].

Outra possibilidade interessante seria colocar o movimento browniano complexo para "passear"por outras superfícies além do plano complexo, como por exemplo em superfícies de Riemann. Neste caso também obtemos resultados interessantes, conforme [davis2][5].

Uma área que tem despertado interesse em pesquisa ultimamente é em Teoria de Campo Conforme através das equações estocásticas de Loewner (veja [lyons1][11]), este pode ser um tema de trabalho futuro para o autor desta dissertação. 


\section{Referências}

[1] A. Atsuji, Nevanlinna Theory via Stochastic Calculus, Jour. of Functional Analysis, 132, pp. 473-510, 1995

[2] R. F. Bass, Probabilistic Techniques in Analysis, Springer-Verlag, 1995

[3] T.K. Carne, Brownian Motion and Nevanlinna Theory, Proc. London Math. Soc., (3), 52, pp. 349-368, 1986

[4] B. Davis, Brownian Motion and Analytic Functions, Ann. Probability, Vol. 7, No. 6, pp. 913-932, 1979

[5] B. Davis, Picard's Theorem and Brownian Motion, Trans. Am. Math. Soc., Vol. 213, pp. 353-362, 1975

[6] R. Durrett, Brownian Motion and Martingales in Analysis, Wadsworth, 1984

[7] R. Durrett, Probability: Theory and Examples, Cambridge, 2010, $4^{\mathrm{a}}$ ed.

[8] A.P. Dvoretsky, P. Erdös, S. Kakutani, Nonincreasing everywhere of the Brownian motion process, Proceedings of the Fourth Berkeley Symposium, vol. II, pp. 103-116, 1961

[9] J.P. Kahane, Brownian Motion and Classical Analysis, Bull. London Math. Soc., 8, pp. 145-155, 1976

[10] A. Lins Neto, Funções de uma Variável Complexa, IMPA, Projeto Euclides, 1993

[11] T.J. Lyons, Brownian Motion in Complex Analysis, notas de aula do curso C10.1b na University of Oxford, 2007, disponível na internet

[12] T.J. Lyons, H. P. McKean, Winding of the Plane Brownian Motion, Advances in Mathematics, 51, pp. 212-225, 1984

[13] F. Niski, Integral Estocástica e Aplicações, dissertação de mestrado em Matemática Aplicada, 2009

[14] R.Paley, N. Wiener, A. Zygmund Note on random functions, Math. Z., 37, pp.647-688, 1933

[15] M. N. Pascu, A Probabilistic Proof of the Fundamental Theorem of Algebra, Proc. Am. Math. Soc., Vol. 133, No. 6, pp. 1707-1711 
[16] W. Rudin, Real and Complex Analysis, McGraw-Hill, 1987, $3^{\mathrm{a}}$ ed.

[17] P. Ruffino, Uma Iniciação aos Sistemas Dinâmicos Estocásticos, IMPA, $27^{\circ}$ Col. Bras. Matem., 2009

[18] S. S. Sheu, Itô's Formula for Complex Semimartingales, disponível na internet

[19] M. Spivak, A Comprehensive Introduction to Differential Geometry, Publish or Perish Inc., Vol. III, pp. 302-310

[20] J. M. Steele, Stochastic Calculus and Financial Applications, SpringerVerlag, 2000 


\section{Índice Remissivo}

adaptada, 30

adaptado, 28

condições de Cauchy-Riemann, 13

condições usuais, 31

contínua à direita, 28

espaço de probabilidade, 17

filtração, 27

função característica, 21

função subharmônica, 16

harmônica, 15

holomorfa, 12

inteira, 12

Lema de Itô, 68

Lema de Itô Complexo, 88

localmente de variação limitada, 68

martingal, 31

martingal complexo, 86

martingal conforme, 87

martingal local, 68

movimento browniano, 49

movimento browniano complexo, 86

movimento browniano padrão, 47

número de passagens de baixo para cima, 35

operadores deslocamento, 51

Pequeno Teorema de Picard, 15

polo de ordem $n, 14$

predizível, 61

Princípio do Máximo Módulo, 14

processo estocástico, 27

processo gaussiano, 27 processo markoviano, 30

processo morto, 27

processo parado, 27

semimartingal, 68

singularidade essencial, 14

singularidade isolada, 13

singularidade removível, 13

submartingal, 31

supermartingal, 31

tempo de parada, 28

Teorema da Aplicação Aberta, 14

Teorema de Lévy, 89

Teorema de Liouville, 15

Teorema de Suporte, 84

Teorema Fundamental da Álgebra, 14

Transformações de Kelvin, 92

uniformemente integrável, 32

variação quadrática, 45 